\title{
The Association of Objectively Measured Physical Activity and Sedentary Behavior with (Instrumental) Activities of Daily Living in Community-Dwelling Older Adults: A Systematic Review
}

\author{
Elvira S Amaral Gomes (D) \\ Keenan A Ramsey (D)' \\ Anna GM Rojer (D) \\ Esmee M Reijnierse (D) ${ }^{2}$ \\ Andrea B Maier ${ }^{1-3}$ \\ 'Department of Human Movement \\ Sciences, @AgeAmsterdam, Faculty of \\ Behavioural and Movement Sciences, \\ Vrije Universiteit Amsterdam, \\ Amsterdam Movement Sciences, \\ Amsterdam, the Netherlands; \\ ${ }^{2}$ Department of Medicine and Aged Care, \\ @AgeMelbourne, The Royal Melbourne \\ Hospital, The University of Melbourne, \\ Melbourne, Victoria, 3050, Australia; \\ ${ }^{3}$ Healthy Longevity Translational \\ Research Program, Yong Loo Lin School \\ of Medicine, National University of \\ Singapore, Singapore; Centre for Healthy \\ Longevity, @AgeSingapore, National \\ University Health System, Singapore
}

\begin{abstract}
Up to $60 \%$ of older adults have a lifestyle characterized by low physical activity (PA) and high sedentary behavior (SB). This can amplify age-related declines in physical and cognitive functions and may therefore affect the ability to complete basic and instrumental activities of daily living (ADL and IADL, respectively), which are essential for independence. This systematic review aims to describe the association of objectively measured PA and SB with ADL and IADL in community-dwelling older adults. Six databases (PubMed, Embase, the Cochrane library, CINAHL, PsychINFO, SPORTDiscuss) were searched from inception to $21 / 06 / 2020$ for articles meeting our eligibility criteria: 1) observational or experimental study, 2) participants' mean/median age $\geq 60$ years, 3) community-dwelling older adults, 4) PA and SB were measured with a(n) accelerometer/pedometer, 5) PA and SB were studied in relation to ADL and/or IADL. Risk of bias was assessed in duplicate using modified versions of the Newcastle-Ottawa scale. Effect direction heat maps provided an overview of associations and standardized regression coefficients $(\beta \mathrm{s})$ were depicted in albatross plots. Thirty articles (6 longitudinal; 24 cross-sectional) were included representing 24,959 (range: 23 to 2749) community-dwelling older adults with mean/median age ranging from 60.0 to 92.3 years (54.6\% female). Higher PA and lower SB were associated with better ability to complete ADL and IADL in all longitudinal studies and overall results of crosssectional studies supported these associations, which underscores the importance of an active lifestyle. The median [interquartile range] of $\beta$ s for associations of PA/SB with ADL and IADL were, respectively, $0.145[0.072,0.280]$ and 0.135 [0.093, 0.211]. Our strategy to address confounding may have suppressed the true relationship of PA and SB with ADL or IADL because of over-adjustment in some included studies. Future research should aim for standardization in PA and SB assessment to unravel dose-response relationships and inform guidelines.
\end{abstract}

Keywords: accelerometry, independent living, aged

\section{Introduction}

Physical activity (PA), defined as bodily movement produced by the contraction of skeletal muscle that requires energy, ${ }^{1}$ has been linked to various health benefits with increasing age. ${ }^{2}$ Up to $60 \%$ of older adults worldwide do not meet PA guidelines ${ }^{3}$ due to physical impairments that arise with aging ${ }^{4,5}$ or sedentary behavior (SB), which refers to waking activity (mainly performed while in a sitting, reclining, or lying posture) with little to no energy expenditure beyond the resting metabolic 
rate. ${ }^{6}$ Low PA (volume, duration, or intensity) and high SB (duration) can be distinct behaviors ${ }^{7}$ that independently amplify age-related decline in many physiological systems $^{8}$ and may therefore affect endurance, muscle strength, and flexibility ${ }^{9}$ as well as cognition. ${ }^{10}$ However, these capacities are necessary to autonomously function in daily life, including engaging in activities of daily living (ADL), referring to self-care tasks, such as transferring in and out of bed, feeding, and dressing, as well as instrumental activities of daily living (IADL), which involve more complex and cognitively demanding tasks, such as housekeeping, shopping, and medication use. ${ }^{11}$

Previous systematic reviews of longitudinal and crosssectional studies have demonstrated that PA classified as of at least moderate intensity is positively associated with the ability to complete ADL and IADL, ${ }^{12,13}$ whereas negative associations were found between SB and the ability to perform these activities. ${ }^{14}$ An important limitation of these findings is that conclusions are predominantly based on self-reported measures of PA and SB (ie, questionnaires), which are especially susceptible in older adult populations to overestimation of $\mathrm{PA}$ and underestimation of $\mathrm{SB}^{15}$ as a result of recall bias. Furthermore, self-reported measures of PA and SB often fail to capture activity at the lower end of the PA continuum, which comprises most of the PA in older adults (eg, light-intensity, short-duration tasks). ${ }^{16} \mathrm{PA}$ and SB can be most accurately quantified with wearable technology (accelerometers, pedometers), which allows for the objective assessment of PA as well as continuous monitoring of activity in daily $\operatorname{life}^{17}$ (ie, frequency, intensity, duration). Objective measurements of PA and SB are therefore essential to advance knowledge by accurately quantifying the association of PA and SB with ADL and IADL, which can ultimately be targeted through public health clinical intervention.

This systematic review aimed to describe the association of objectively measured PA and SB with ADL and IADL in community-dwelling older adults.

\section{Materials and Methods}

The protocol of this review was registered in the PROSPERO International prospective register of systematic reviews with registration number CRD42018103910.

\section{Information Sources and Search Strategy}

Two assessors (the Vrije Universiteit librarian (RO) and AR) conducted a systematic literature search based on the Preferred Reporting Items for Systematic Reviews and
Meta-Analysis (PRISMA) statement, ${ }^{18}$ consulting the following electronic databases from inception to June 21, 2020: PubMed, Embase, the Cochrane Library (via Wiley), CINAHL, PsychINFO, and SPORTDiscuss (via EBSCO). The search terms "active or inactive lifestyle", "motor activity", and "people over 60 years of age" were used to ascertain articles that studied PA and SB in relation to any health outcome in older adults; the full search strategy is presented in Appendix A. Articles that reported associations of $\mathrm{PA}$ and $\mathrm{SB}$ with $\mathrm{ADL}$ and IADL were organized and managed in the software Endnote (Version X8.2 Clarivate Analytics, Philadelphia, USA) and Rayyan QRCI. $^{19}$

\section{Inclusion Criteria}

Full-text articles published in English or Dutch were considered eligible for this systematic review based on the following criteria: 1) observational or experimental study, 2) participants' mean or median age $\geq 60$ years old, 3 ) study population consisted of community-dwelling older adults, 4) PA and SB were measured with an accelerometer or pedometer, 5) ADL was defined as any tool or questionnaire explicitly described as measuring ADL and/ or IADL, and 6) PA and SB were studied in relation to ADL and/or IADL. For intervention studies, associations at baseline or control group data were included.

\section{Article Selection}

Search results were assessed for possible eligibility based on title and abstract screening by two independent assessors ( $K R$ and EvdR) using the Rayyan screening software. ${ }^{19}$ Full-text screening was performed in duplicate by two independent assessors (KR and $\mathrm{LD}$, or $\mathrm{AR}$ ) and differences in opinion with regard to inclusion and exclusion decisions were resolved by another assessor (AM). The references of all included articles were screened for additional eligible articles.

\section{Data Extraction}

Data extraction was performed by two independent assessors (EG and WZ) and disagreement was settled by a third assessor (KR). The following data were extracted: first author; year of publication; country; cohort; study design with, if applicable, follow-up period; characteristics of study population (population selection), sample size, age (in years), sex (number and percentage of females), device used for objective assessment of PA/SB (accelerometer, pedometer), device name, wearing location of device, 
number of monitor days, mean device wear time, minimum duration of device wear to define a valid day, number of valid days required for analysis, reported measures of $\mathrm{PA} / \mathrm{SB}$ and their definitions, PA/SB scores, tools and definitions used for ADL and IADL assessment, activities included in an ADL or IADL tool/questionnaire, ADL/ IADL scores, adjustment model(s), statistical analysis to study association(s), effect size(s) with 95\% confidence interval $(95 \% \mathrm{CI})$ or standard error (SE), and significance level (p-value).

\section{Assessment of Study Quality}

Study quality and risk of bias were assessed by two independent assessors (EG and WZ) using modified versions of the Newcastle-Ottawa scale (NOS) for cross-sectional and longitudinal studies, ${ }^{20}$ customized for this systematic review. Three domains, selection (representativeness of study cohort and ascertainment of exposure), comparability (adjustment model(s) and statistical analysis), and outcome (assessment of outcome and, if applicable, adequacy to follow-up), were assessed and the median of total possible stars (points) was set as the cut-off to determine high or low quality, defined as $\geq$ or $<4$ out of 7 and $\geq$ or $<5$ out of 9 for cross-sectional and longitudinal studies, respectively (Appendix B).

\section{Data Analysis and Visualization}

Extracted information and associations between PA/SB and ADL or IADL were reported in tables, visualized in effect direction heat maps, ${ }^{21}$ and synthesized in albatross plots $^{22}$ according to the PRISMA ${ }^{18}$ and Synthesis Without Meta-analysis $(\mathrm{SWiM})^{23}$ guidelines. Data were reported based on the following hierarchy of adjustment: 1) age and sex, 2) age and sex, and other factors (eg, cognitive function, number of chronic diseases, body mass index), 3) age or sex, and other factors, 4) other factors only, and 5) unadjusted (crude) model. When articles reported more than one type of statistical analysis for an association, the following hierarchy for reporting was considered: 1) adjusted linear regression, 2) adjusted logistic regression, 3) partial correlation, 4) unadjusted linear regression (including Pearson's and Spearman correlation), 5) analysis of variance (ANOVA), and 6) Mann-Whitney test, Student's $t$-test, or chi-squared test. Continuous measures of PA/SB were used if reported and categorical variables were used otherwise. P-values were calculated when these were not reported: for linear regression: the upper and lower limit of the $95 \%$ CI were used to acquire the SE,
$\mathrm{SE}=(($ upper limit of $95 \% \mathrm{CI}$ - lower limit of $95 \% \mathrm{CI}) /$ $(2 * 1.96))$, which was then used to obtain the absolute value (abs) of the $\mathrm{z}$-statistic $(\mathrm{z}), \mathrm{z}=\mathrm{abs}$ (regression coefficient/SE), and eventually calculate the $\mathrm{p}$-value, $\mathrm{p}$ (calc) $=\exp \left(\left(-0.717^{*} z\right)-\left(0.416^{*}\left(z^{2}\right)\right)\right)$. The aforementioned formulae were also used for ratio measures (odds ratio (OR), hazard ratio (HR), and risk ratio (RR)), except for that the upper and lower limits of the $95 \%$ and effect sizes were transformed into logarithms using natural $\log (\ln )$ first. $^{24}$ For correlations, sample size (n) and coefficients (including Pearson's R and Spearman's Rho) were used to calculate the t-statistic ( $t), t=R * \sqrt{ }((n-2) /(1 / R))$, of which the absolute value (abs) was compared to the two-sided student's t-distribution using T.VERD.2T in Microsoft Excel to obtain $\mathrm{p}$ (calc). For comparison between groups, mean values and standard deviations $(\mathrm{sd})$ were used to calculate $\mathrm{t}, \mathrm{t}=\left(\left(\operatorname{mean}_{1}-\right.\right.$ mean $\left._{2}\right) / \sqrt{ }\left(\left(\left(\mathrm{n}_{1}-1\right) *\left(\left(\mathrm{sd}_{1}\right)^{2}\right)\right)+\left(\left(\mathrm{n}_{2}-1\right) *\left(\left(\mathrm{sd}_{2}\right)^{2}\right)\right)\right) /$ $\left.\left(\left(\mathrm{n}_{1}+\mathrm{n}_{2}-2\right) *\left(\left(1 / \mathrm{n}_{1}\right)+\left(1 / \mathrm{n}_{2}\right)\right)\right)\right)$, which was then compared to the two-sided t-distribution using the earlier-mentioned function in Microsoft Excel. Associations with p-values that could not be calculated were conservatively estimated for effect direction heat maps as being $\geq 0.25$ or the largest p-value derived from the reported information and excluded from albatross plots. ${ }^{25}$

\section{Effect Direction Heat Maps}

Effect direction heat maps ${ }^{21}$ were created to provide a qualitative overview of all associations between PA/SB measures and ADL or IADL and were stratified by study design (longitudinal versus cross-sectional) and ordered by sample size. Articles that included combined measures of ADL and IADL were categorized as IADL because inability to carry out more complex and cognitively demanding activities precedes difficulty in ADL. ${ }^{26}$ The observed direction of effect was determined based on whether higher PA and lower SB were associated with better (positive effect) or worse (negative effect) ADL and IADL, indicated by an upwards or downwards triangle, respectively. The following color scheme was used to present significance: $\mathrm{p}<0.001$ (dark blue filled triangle), $0.001 \leq \mathrm{p}<0.01$ (blue filled triangle), $0.01 \leq \mathrm{p}<0.05$ (light blue filled triangle), $0.05 \leq \mathrm{p}<0.1$ (light grey empty triangle), $0.01 \leq \mathrm{p}<0.25$ (grey empty triangle), and $\mathrm{p} \geq 0.25$ (dark grey empty triangle).

\section{Albatross Plots}

Albatross plots are scatter plots of sample size plotted against two-sided p-values, stratified by the observed 
effect direction to graphically present the estimated magnitude of associations ${ }^{22}$ (expressed as median with corresponding interquartile range, [IQR]). Each data point represents an association and based on whether higher PA and lower SB were associated with better (positive effect) or worse (negative effect) ADL and IADL, data points fall on the right or left side of albatross plots, respectively. Contour lines were superimposed on the plot to examine hypothetical effect sizes, here selected as standardized regression coefficients $(\beta s)$, and derived from the following equation: $\mathrm{N}=\left(\left(\left(1-\beta^{2}\right) / \beta^{2}\right)^{*}\left(Z_{\mathrm{p}}\right)^{2}\right)$ in which $Z_{\mathrm{p}}$ denotes the $\mathrm{z}$-value associated with given two-sided $p$-values. Separate albatross plots were made for ADL and IADL using the Stata Statistical Software, Release 16.0 (StataCorp LLC, College Station, Texas, United States), each stratified by measures of PA and SB. Sensitivity analyses were performed by stratifying albatross plots using population selection (disease versus general), study design (cross-sectional versus longitudinal), adjustment (adjusted versus unadjusted associations), device type (accelerometer versus pedometer), and device wearing location. For the latter sensitivity analysis, device wearing locations were entered into the albatross plots if reported for $\geq 5$ associations to obtain an IQR.

\section{Results}

The literature search identified 18,806 articles of which 9660 articles were left after duplicate removal. Of the 1017 full texts assessed for eligibility, 30 articles ${ }^{27-56}$ were included in this systematic review (Figure 1).

\section{Characteristics of Studies}

A total of 24,959 (range: 23 to 3749) community-dwelling older adults were included with mean or median age ranging from 60.0 to 92.3 years and, on average, populations were $54.6 \%$ female. In 11 articles, specific disease groups were studied: osteoarthritis (OA), ${ }^{34,36,44,54}$ chronic obstructive pulmonary disease (COPD), ${ }^{28,39,45,56}$ cirrhosis, ${ }^{37}$ Parkinson's disease, ${ }^{38}$ and stroke survivors. ${ }^{40}$ Longitudinal associations were reported in six articles ${ }^{27,31,34,36,53,54}$ (mean follow-up period of 3.1 years) and represented 7554 older adults with mean or median age ranging from 62.4 to 80.6 years (56.8\% female); remaining articles reported cross-sectional associations (Table 1). The NOS categorized 26 out of 30 articles as high quality (Table 2 ).

\section{Measures of Physical Activity and Sedentary Behavior}

Accelerometers were used in 28 studies, while two studies27,28 used pedometers to objectively measure $\mathrm{PA} / \mathrm{SB}$ (Table 3). The following measures of PA/SB were included: number of steps (or walking duration), $27,28,37,38,41,44,45,50,55$ activity counts (or accelerations, movement intensity),$29,33,42,43,45,49,53,55,56$ energy expenditure (EE), ${ }^{31,37,45,50}$ duration (in different units of time) of total PA (TPA) (or mobile duration), ${ }^{45,47,51,56}$ moderate to vigorous PA (MVPA) (or moderate PA (MPA) or vigorous PA (VPA) individual), ${ }^{30-32,34,36-40,46-49,51,52,54,55}$ light PA (LPA), ${ }^{34,40,47,49,52,55}$ and SB (or lying duration, immobile time),${ }^{30-32,35,37,38,40,43,45,47,49,52,55}$ breaks per sedentary hour (SB break rate), ${ }^{52}$ and breaks in sedentary time (BST). ${ }^{32,52,55}$

\section{Assessment of Activities of Daily Living and Instrumental Activities of Daily Living} The association of PA/SB measures and ADL was studied in 20 articles using the following tools: London Chest Activities of Daily Living (LCADL) scale, ${ }^{28,39}$ Katz Index of Independence in Activities of Daily Living (Katz), ${ }^{29,53}$ Glittre-ADL test, ${ }^{45}$ Western Ontario and McMaster Universities osteoarthritis index (WOMAC) functional limitation sub-scale, ${ }^{27}$ Health Assessment Questionnaire Disability Index (HAQ-DI), ${ }^{49}$ Barthel Index, ${ }^{40}$ Composite Physical Function (CPF) scale, ${ }^{52}$ Knee injury and Osteoarthritis Outcome Score (KOOS) questionnaire function in daily life sub-scale, ${ }^{44}$ Parkinson's Disease Questionnaire-39 (PDQ-39) activities of daily living dimension, ${ }^{38}$ Nottingham Extended Activities of Daily Living (NEADI), ${ }^{56}$ and custom questionnaires $^{30,31,35,36,43,48,50,51,55}$ (Table 4). In 13 articles, the association between measures of PA/SB and IADL was studied with the use of the following tools: Tokyo Metropolitan Institute of Gerontology Index of Competence (TMIG), ${ }^{32}$ Rosow-Breslau scale, ${ }^{37} \mathrm{CPF}$ scale, ${ }^{47,52}$ Late-Life Disability Index (LLDI) ${ }^{54}$ Late-Life Function and Disability Index (LLFDI), ${ }^{40-42,46}$ and custom questionnaires ${ }^{31,33,34,43}$ (Table 5).

\section{Associations of Physical Activity and Sedentary Behavior with Activities of Daily Living and Instrumental Activities of Daily Living}

All associations are visualized by effect direction heat maps (Figure 2), standardized regression coefficients ( $\beta \mathrm{s}$ ) for each association are presented by albatross plo ts 


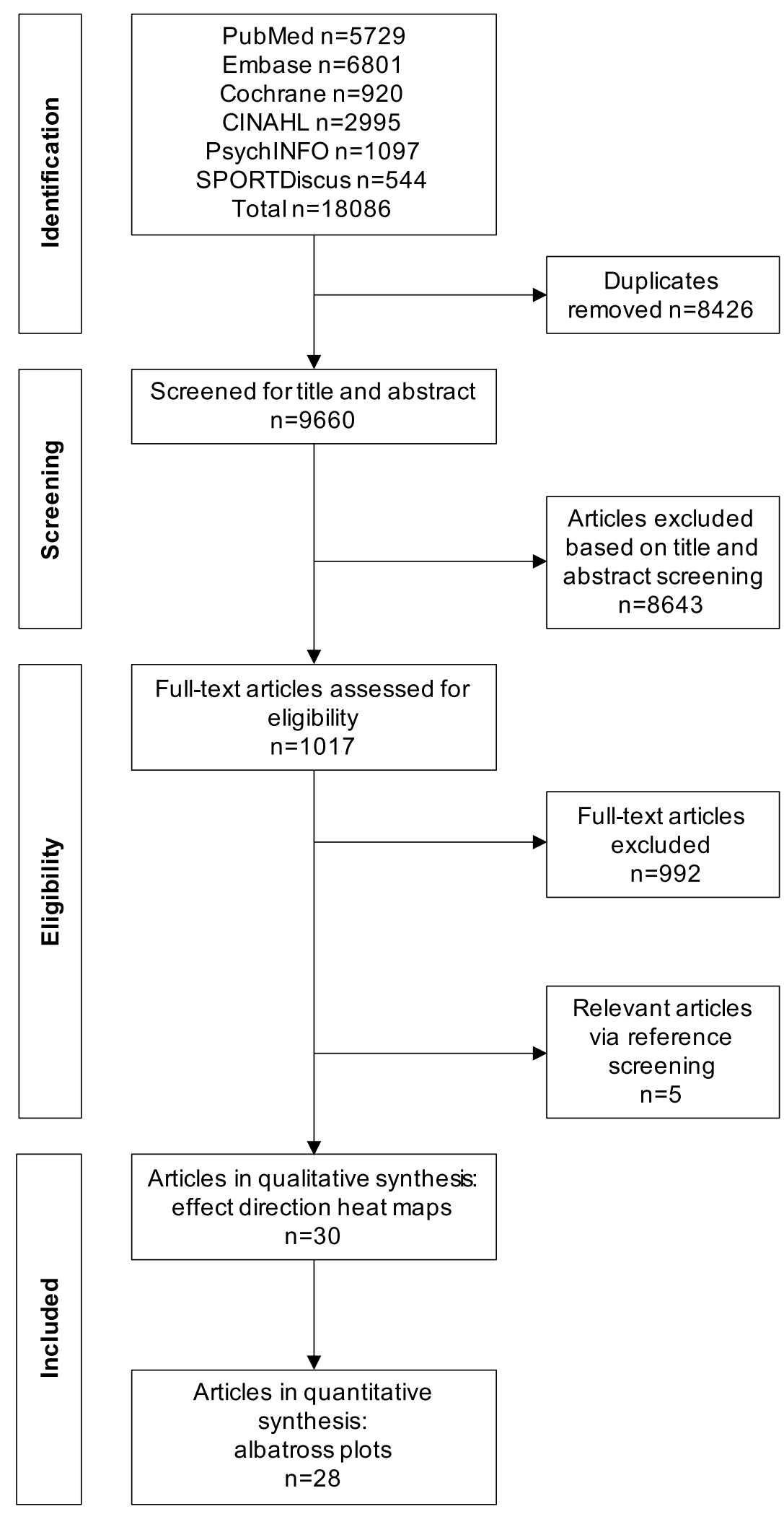

Figure I Flowchart of article selection process. 


\begin{tabular}{|c|c|c|c|c|c|c|c|c|c|c|c|c|c|}
\hline 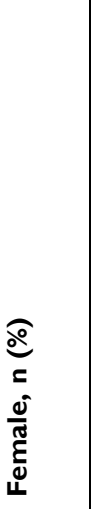 & 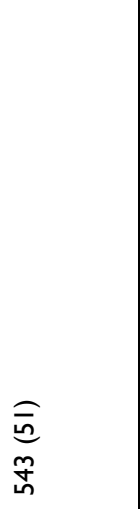 & 0 & 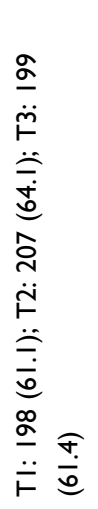 & 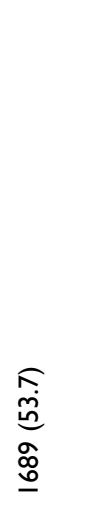 & 0 & & 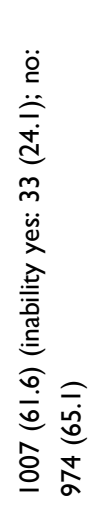 & 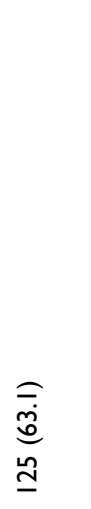 & 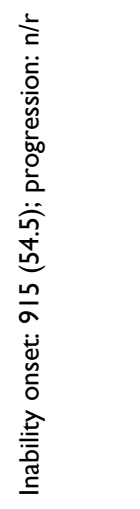 & 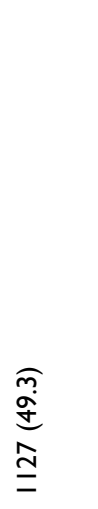 & $\begin{array}{l}\widehat{O} \\
\stackrel{0}{n} \\
\stackrel{0}{0} \\
\infty \\
\ddot{\bar{\alpha}}\end{array}$ & $\grave{c}$ & 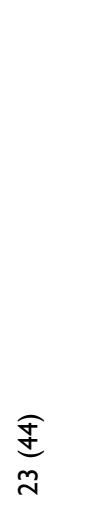 \\
\hline 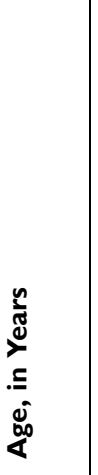 & $\begin{array}{l}\stackrel{+}{+} \\
+1 \\
\tilde{0}\end{array}$ & \begin{tabular}{l}
0 \\
$\circ$ \\
+1 \\
\multirow{1}{0}{} \\
\end{tabular} & 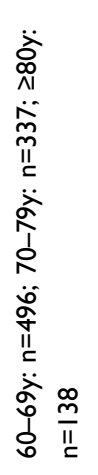 & 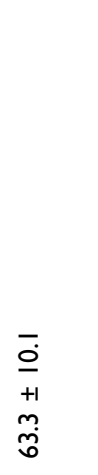 & 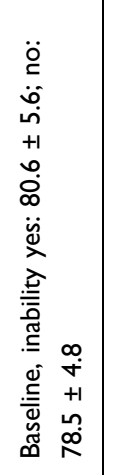 & $\begin{array}{l}\infty \\
\dot{+} \\
+1 \\
\dot{m} \\
\stackrel{\sim}{\grave{u}}\end{array}$ & 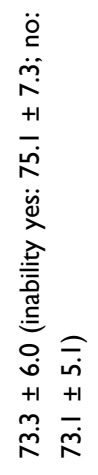 & 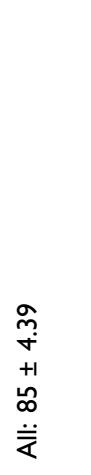 & 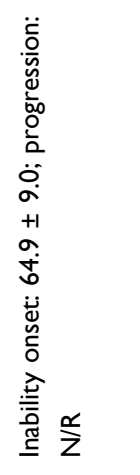 & $\grave{c}$ & 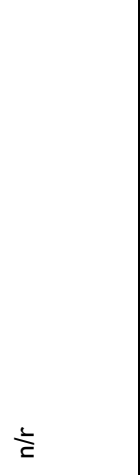 & 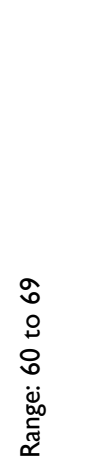 & 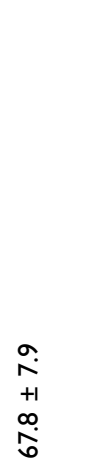 \\
\hline 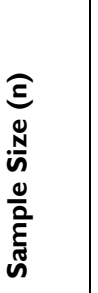 & 호 & 员 & 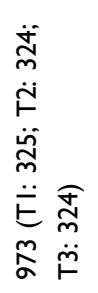 & $\frac{f}{m}$ & 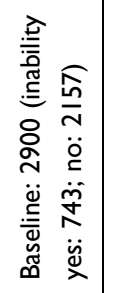 & 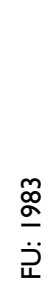 & 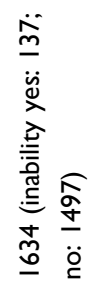 & 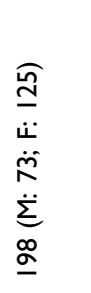 & 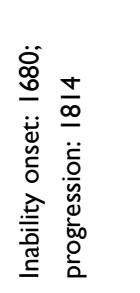 & $\underset{\sim}{\stackrel{\sim}{\sim}}$ & 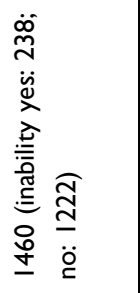 & n̊n & 稃 \\
\hline 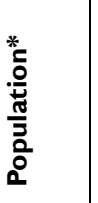 & I & 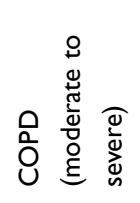 & | & 1 & I & & I & 1 & 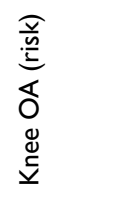 & 1 & 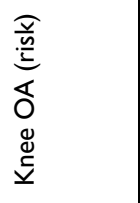 & 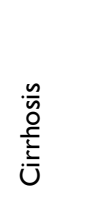 & 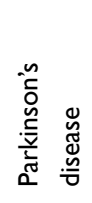 \\
\hline 䓂点 & 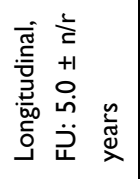 & 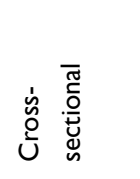 & 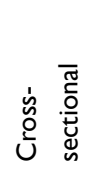 & 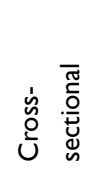 & 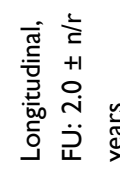 & & 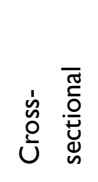 & 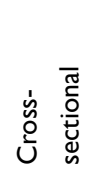 & 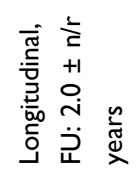 & 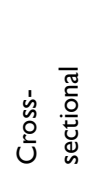 & 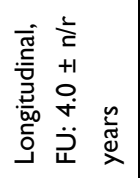 & 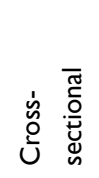 & 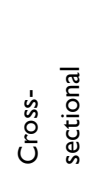 \\
\hline $\begin{array}{l}\frac{t}{0} \\
\frac{0}{0}\end{array}$ & 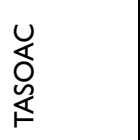 & $\stackrel{\pi}{\underline{c}}$ & 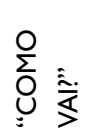 & $\begin{array}{l}\text { 岁 } \\
\text { 预 } \\
\text { Z }\end{array}$ & $\begin{array}{l}\text { ô } \\
\frac{1}{\Sigma}\end{array}$ & & 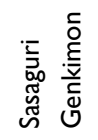 & 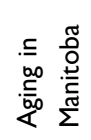 & 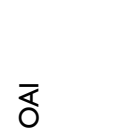 & $\begin{array}{l}\text { 㟧 } \\
\text { 至 } \\
\text { Z }\end{array}$ & $\bar{\delta}$ & $\stackrel{\pi}{\Omega}$ & $\stackrel{\mathscr{n}}{\mathrm{C}}$ \\
\hline 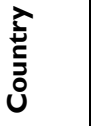 & $\stackrel{2}{\gtrless}$ & \llcorner & 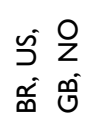 & $\overleftarrow{U}$ & $\begin{array}{l}\text { U } \\
\text { vi }\end{array}$ & & 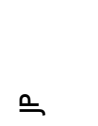 & $\overleftarrow{U}$ & $\leftrightharpoons$ & $\widetilde{د}$ & 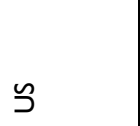 & 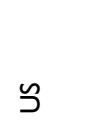 & 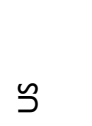 \\
\hline 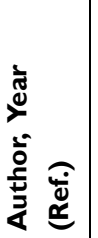 & 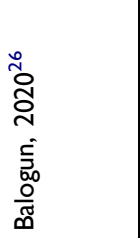 & 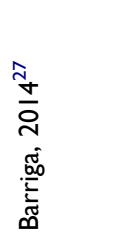 & 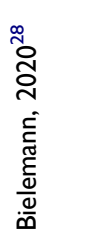 & 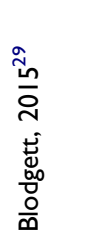 & 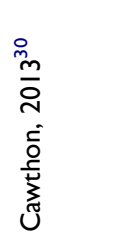 & & 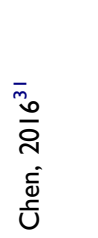 & 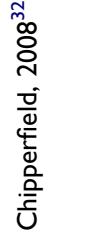 & 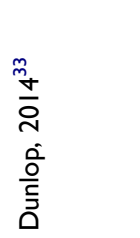 & 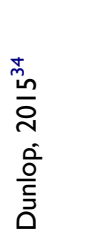 & 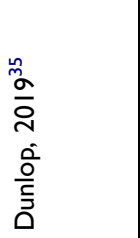 & 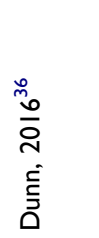 & 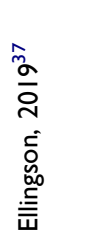 \\
\hline
\end{tabular}




\begin{tabular}{|c|c|c|c|c|c|c|c|c|c|c|c|c|}
\hline $\begin{array}{l}\hat{\widetilde{m}} \\
\infty \\
\stackrel{m}{1} \\
\ddot{\bar{\kappa}}\end{array}$ & $\begin{array}{l}\underset{\tilde{N}}{\tilde{N}} \\
\underset{\sim}{N}\end{array}$ & 음 & 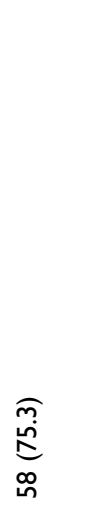 & 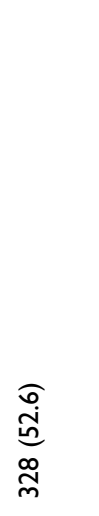 & $\begin{array}{l}\widehat{f} \\
\stackrel{d}{0} \\
\hat{4}\end{array}$ & $\begin{array}{l}\widehat{\overline{\mathrm{i}}} \\
\underline{\underline{0}}\end{array}$ & 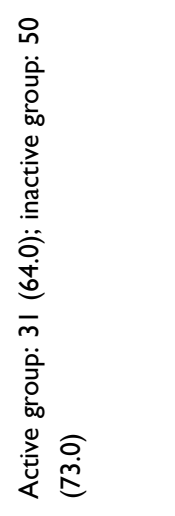 & 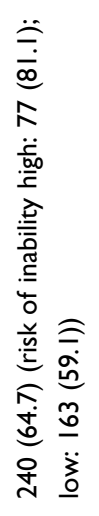 & 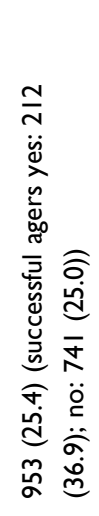 & 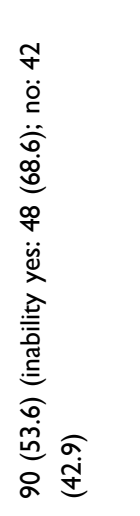 & 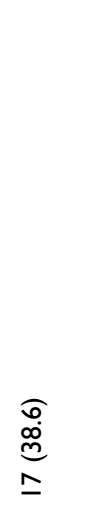 & 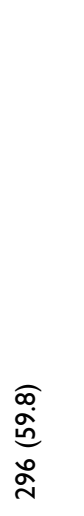 \\
\hline 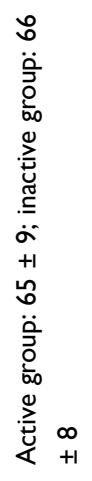 & $\begin{array}{l}\stackrel{\sim}{=} \\
\overline{+1} \\
\infty \\
\underline{0}\end{array}$ & 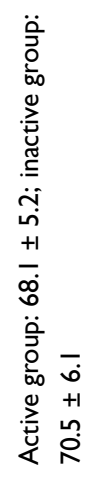 & $\begin{array}{l}\hat{i} \\
+1 \\
+ \\
\stackrel{1}{r}\end{array}$ & 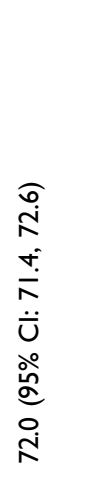 & $\begin{array}{l}\stackrel{0}{0} \\
\dot{m} \\
+1 \\
m \\
0 \\
0\end{array}$ & $\begin{array}{l}n \\
+1 \\
n \\
0\end{array}$ & 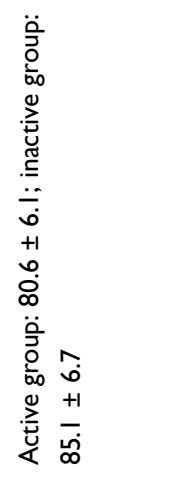 & 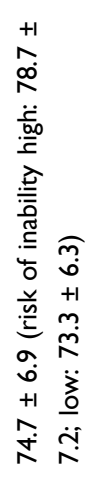 & 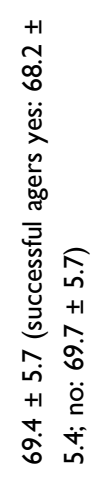 & 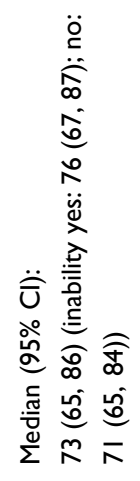 & 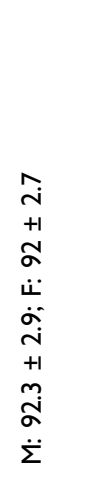 & 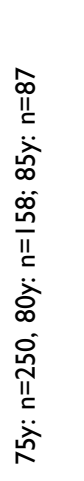 \\
\hline 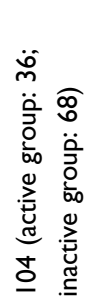 & 이 & 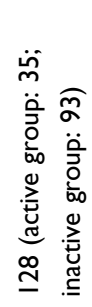 & $\stackrel{\infty}{\sim}$ & స్ర & กี & $\stackrel{\infty}{m}$ & 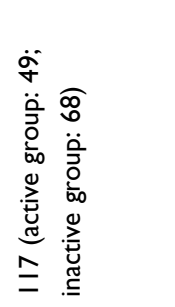 & 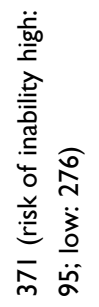 & 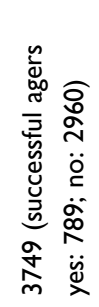 & 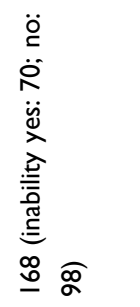 & $\begin{array}{l}E \\
\ddot{L} \\
\ddot{N} \\
\ddot{\Sigma} \\
\dot{J} \\
\dot{J}\end{array}$ & 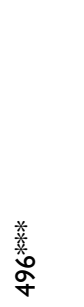 \\
\hline $\begin{array}{l}0 \\
\text { ò }\end{array}$ & 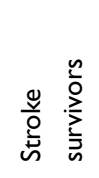 & 1 & 1 & I & 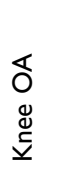 & 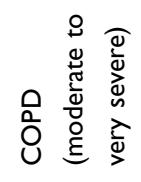 & 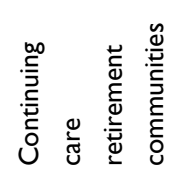 & I & I & I & I & I \\
\hline 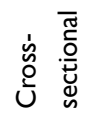 & 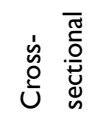 & 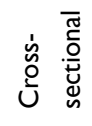 & 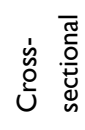 & 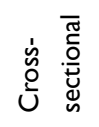 & 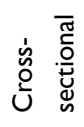 & 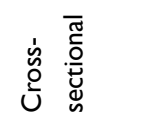 & 恉 & 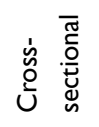 & 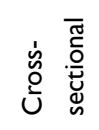 & 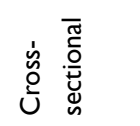 & 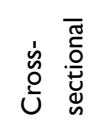 & 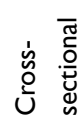 \\
\hline$\stackrel{\pi}{c}$ & $\stackrel{\pi}{\varepsilon}$ & $\stackrel{\pi}{\Omega}$ & $\stackrel{\pi}{\Xi}$ & $\begin{array}{l}\frac{0}{5} \\
\frac{1}{5} \\
Z\end{array}$ & $\stackrel{\pi}{\mathrm{c}}$ & $\stackrel{\pi}{c}$ & $\stackrel{\pi}{\mathfrak{c}}$ & $\stackrel{\pi}{\varepsilon}$ & 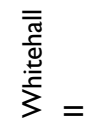 & 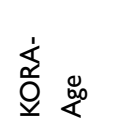 & $\stackrel{\pi}{\varrho}$ & 岕 \\
\hline ऽ & 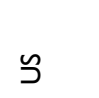 & 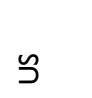 & ऽ & $\breve{د}$ & $\stackrel{\mathscr{x}}{\underline{x}}$ & $\stackrel{\mathscr{c}}{\boldsymbol{D}}$ & $\breve{s}$ & t & $\begin{array}{l}\hat{z} \\
\text { 占 }\end{array}$ & 㟔 & 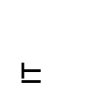 & च \\
\hline 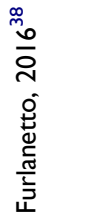 & 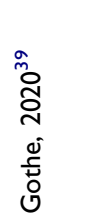 & $\begin{array}{l}\frac{+}{0} \\
\frac{0}{2} \\
\dot{\bar{T}} \\
\text { I }\end{array}$ & 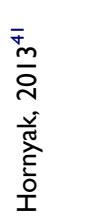 & 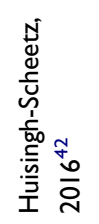 & 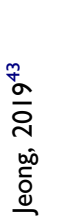 & 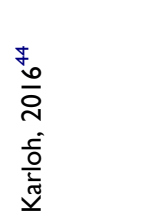 & 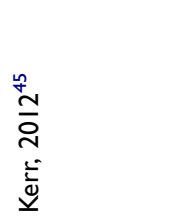 & 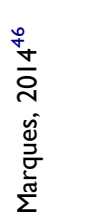 & 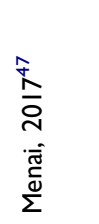 & 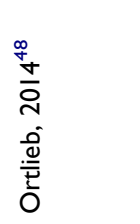 & 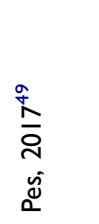 & 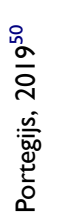 \\
\hline
\end{tabular}




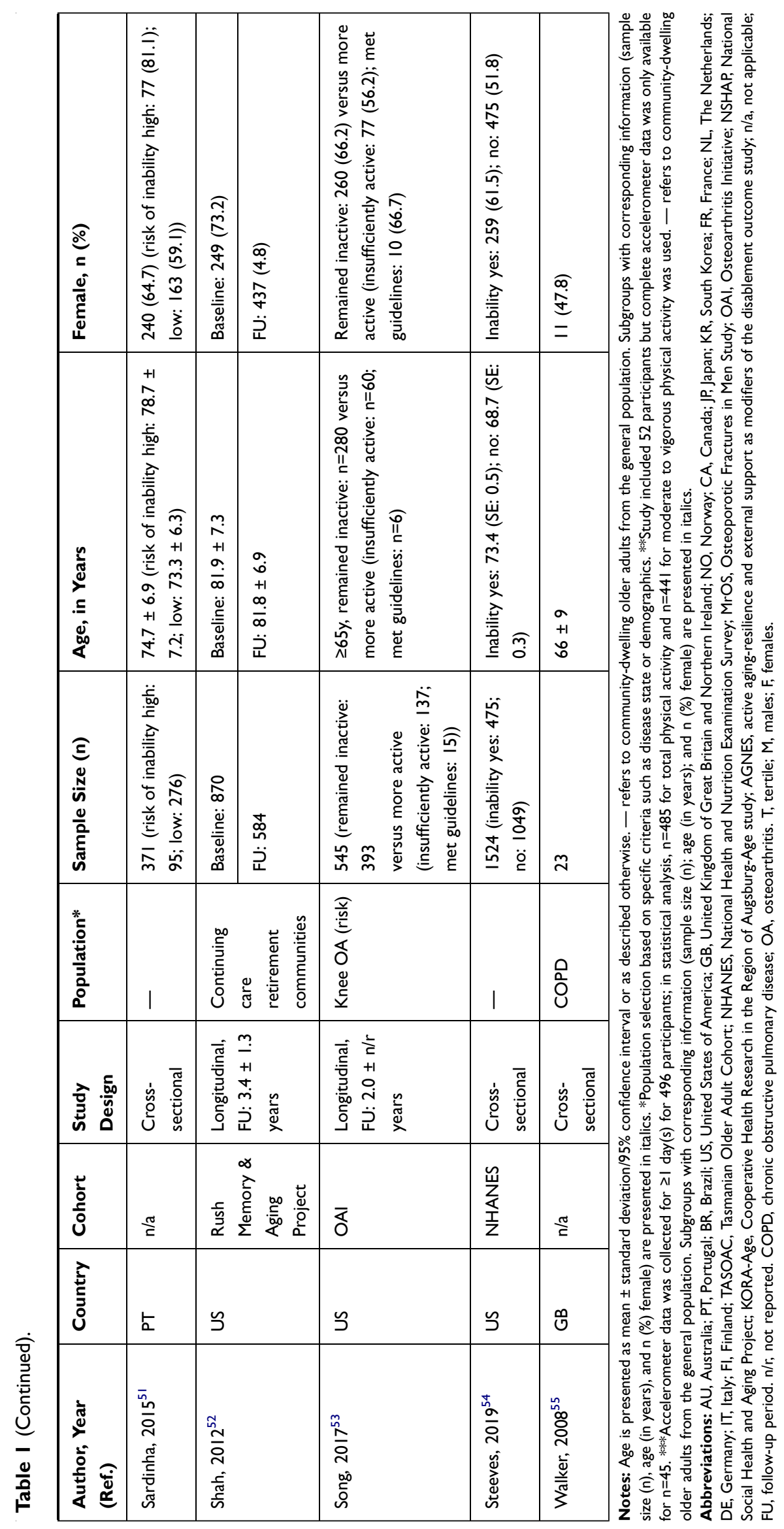




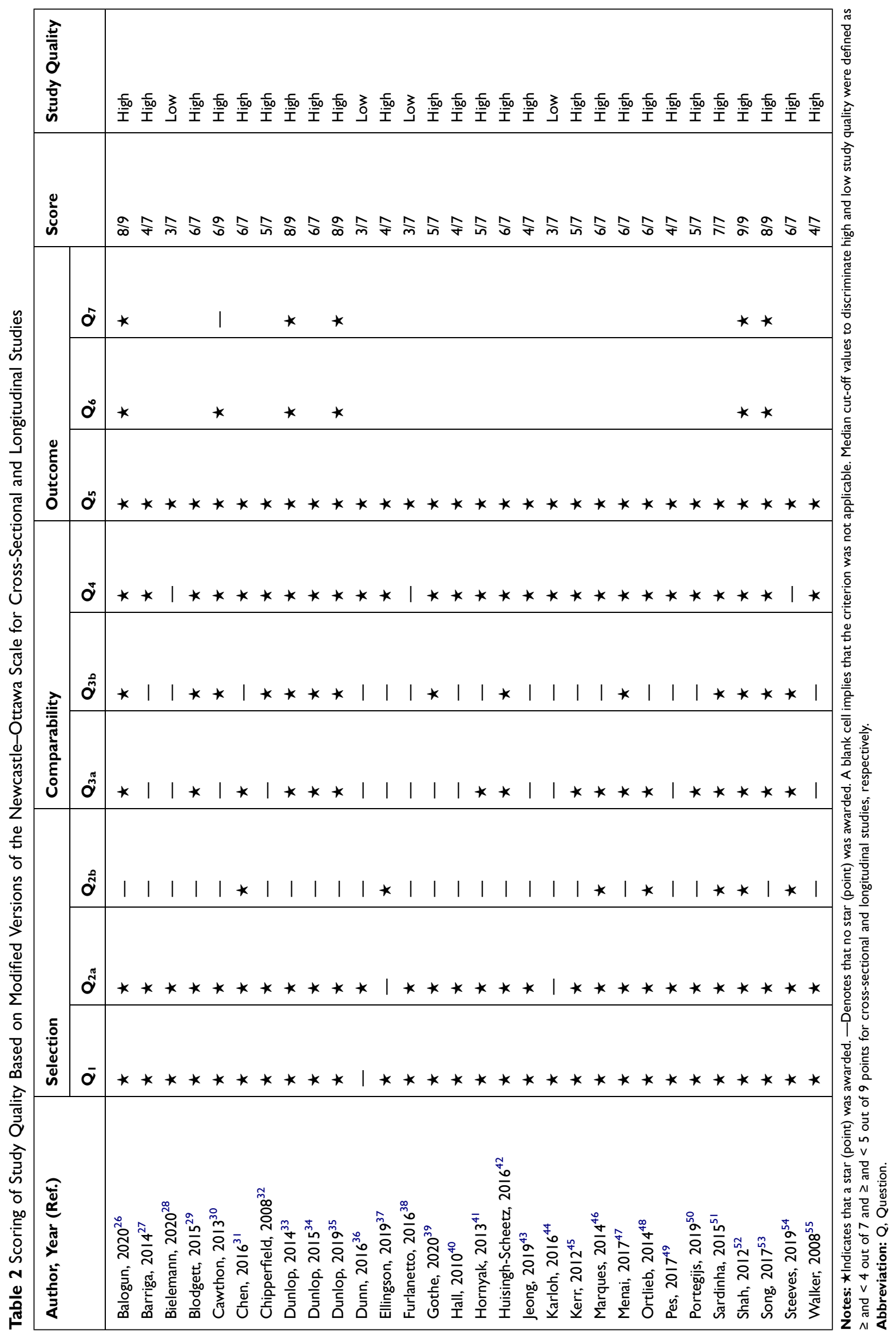




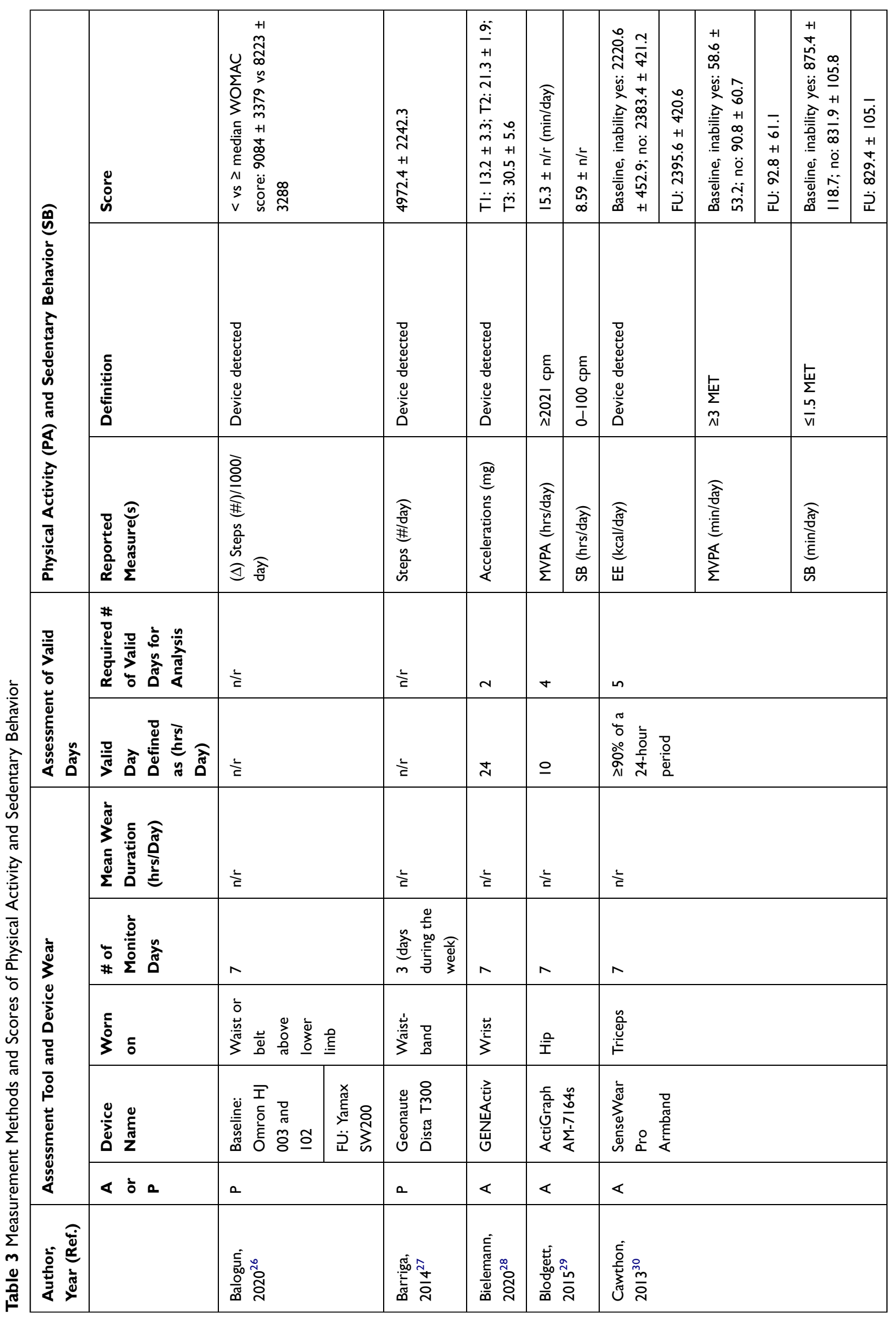




\begin{tabular}{|c|c|c|c|c|c|c|c|c|c|c|c|c|c|c|}
\hline 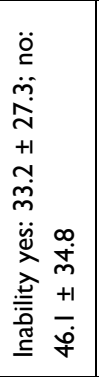 & 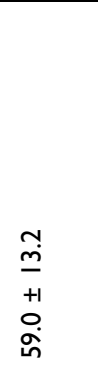 & 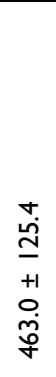 & 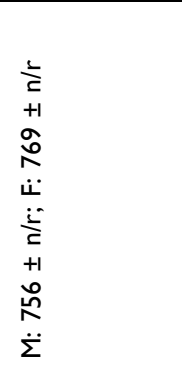 & 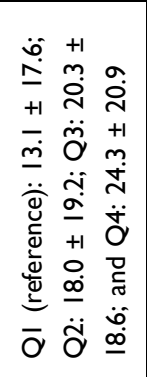 & 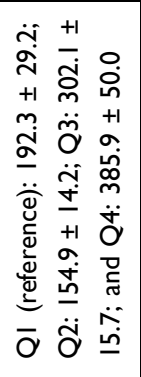 & $\begin{array}{l}a \\
a \\
+1 \\
\sigma \\
\infty\end{array}$ & 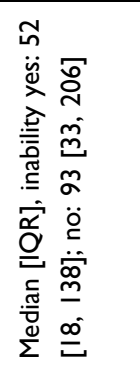 & 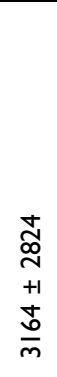 & $\begin{array}{l}\stackrel{0}{f} \\
+1 \\
\infty \\
\underset{\sim}{\sim}\end{array}$ & $\mid \begin{array}{c}a \\
\dot{b} \\
+1 \\
a \\
\dot{\sigma}\end{array}$ & $\begin{array}{l}o \\
\infty \\
\infty \\
+1 \\
\sigma \\
\dot{n}\end{array}$ & 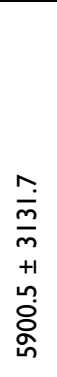 & 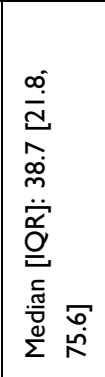 & $\begin{array}{c} \\
\bar{i} \\
+1 \\
+\infty \\
+\infty\end{array}$ \\
\hline 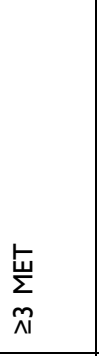 & 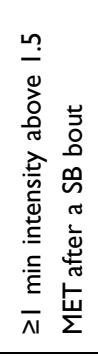 & 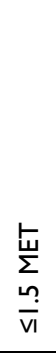 & 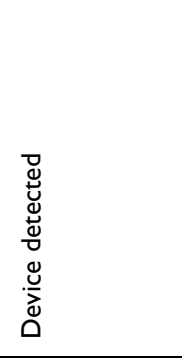 & 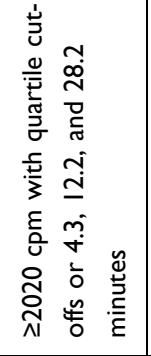 & 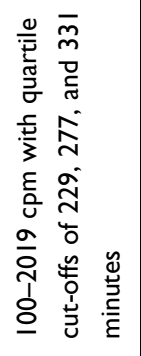 & $\begin{array}{l}\frac{\varepsilon}{0} \\
\frac{o}{v}\end{array}$ & 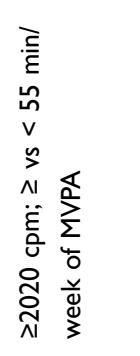 & 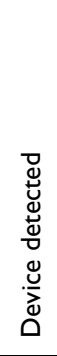 & 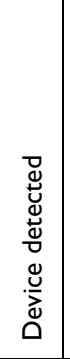 & 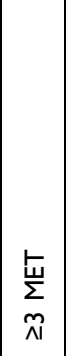 & 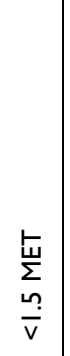 & 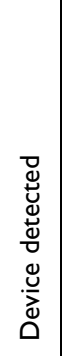 & $\grave{c}$ & 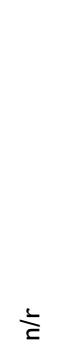 \\
\hline 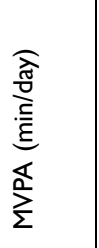 & 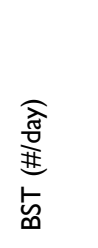 & 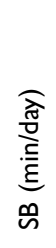 & 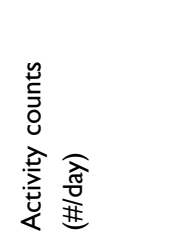 & 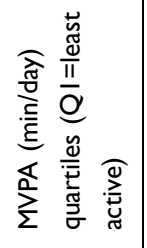 & 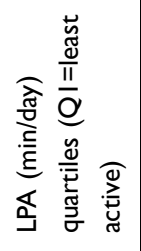 & 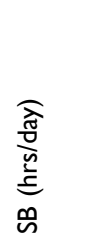 & 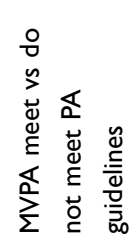 & 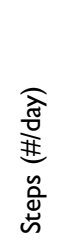 & 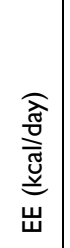 & 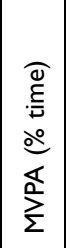 & 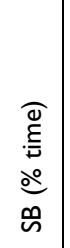 & 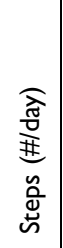 & 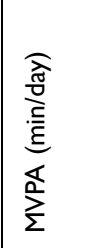 & 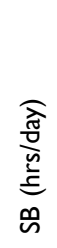 \\
\hline+ & & & - & $\sigma$ & & $\sigma$ & $\sigma$ & $\sigma$ & & & & \multicolumn{3}{|c|}{ 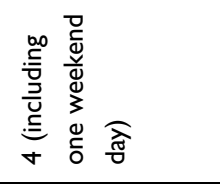 } \\
\hline 으 & & & $\grave{c}$ & 으 & & 으 & 으 & 으 & & & & \multicolumn{3}{|l|}{ 으 } \\
\hline $\begin{array}{l}\infty \\
\stackrel{\infty}{+} \\
+1 \\
0 \\
\dot{ \pm}\end{array}$ & & & 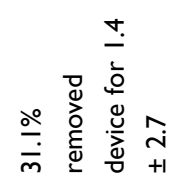 & 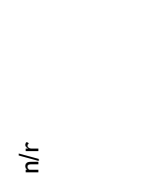 & & $\grave{c}$ & 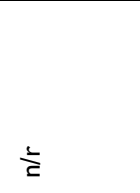 & 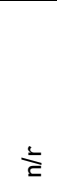 & & & & \multicolumn{3}{|l|}{$\begin{array}{l}\stackrel{0}{-} \\
+1 \\
m \\
\stackrel{+}{ \pm}\end{array}$} \\
\hline r & & & - & r & & $\wedge$ & r & r & & & & \multicolumn{3}{|l|}{$\wedge$} \\
\hline 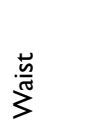 & & & $\frac{\vec{n}}{\sum^{\frac{1}{3}}}$ & $\stackrel{\varrho}{\bar{I}}$ & & $\stackrel{\stackrel{ }{\bar{I}}}{ }$ & 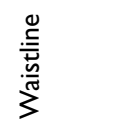 & 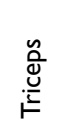 & & & & \multicolumn{3}{|c|}{ 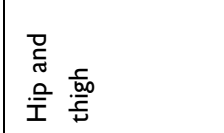 } \\
\hline 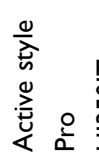 & & & 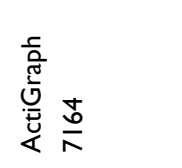 & 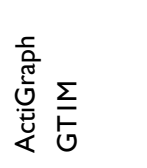 & & 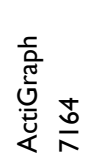 & 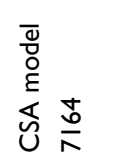 & & है & & & \multicolumn{3}{|c|}{ 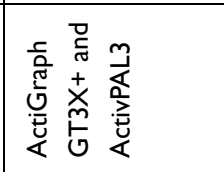 } \\
\hline$\ll$ & & & $\ll$ & $\varangle$ & & $\varangle$ & $\varangle$ & $\ll$ & & & & \multicolumn{3}{|l|}{$\varangle$} \\
\hline 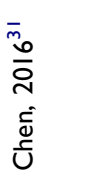 & & & 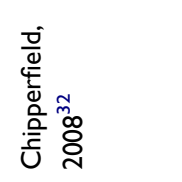 & 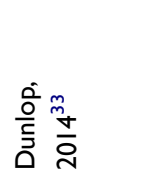 & & 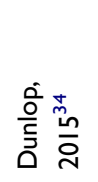 & 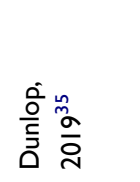 & 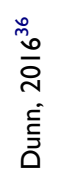 & & & & \multicolumn{3}{|c|}{ 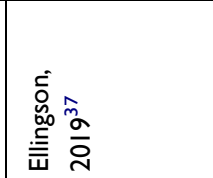 } \\
\hline
\end{tabular}




\begin{tabular}{|c|c|c|c|c|c|c|c|c|c|c|}
\hline \multirow{3}{*}{ 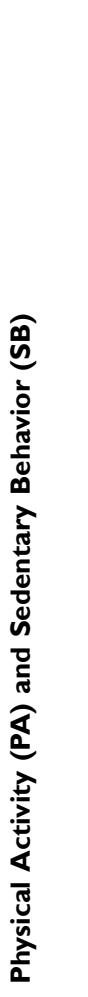 } & ڤัँ & 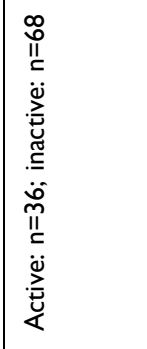 & $\begin{array}{l}\stackrel{1}{=} \\
+1 \\
\stackrel{1}{n}\end{array}$ & $\begin{array}{l}+\dot{a} \\
\bar{\sigma} \\
+1 \\
m \\
\tilde{c} \\
\tilde{n}\end{array}$ & $\begin{array}{l}0 \\
o \\
o \\
o \\
+1 \\
\omega \\
\tilde{\omega} \\
0 \\
0\end{array}$ & 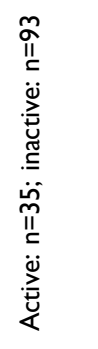 & 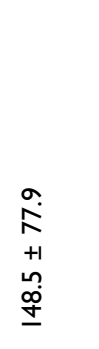 & 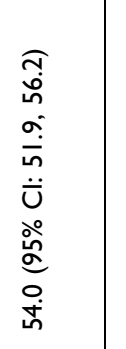 & 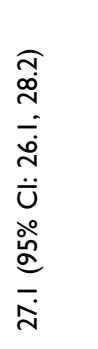 & $\begin{array}{l}\infty \\
\stackrel{\infty}{+} \\
\dot{0} \\
m \\
+1 \\
o \\
\stackrel{0}{0} \\
\alpha\end{array}$ \\
\hline & 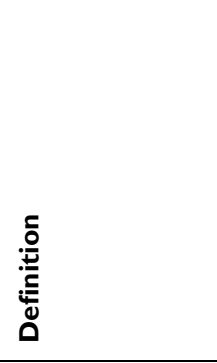 & 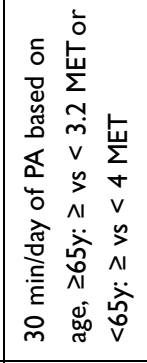 & 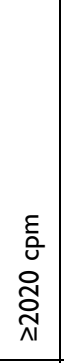 & $\begin{array}{l}\varepsilon \\
\underline{0} \\
\frac{0}{0} \\
\frac{1}{0} \\
\frac{1}{0}\end{array}$ & $\begin{array}{l}\frac{\varepsilon}{0} \\
\frac{0}{v} \\
\frac{8}{v 1}\end{array}$ & 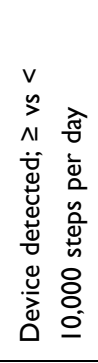 & 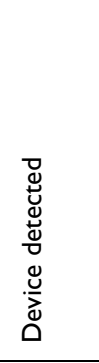 & 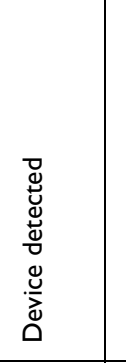 & 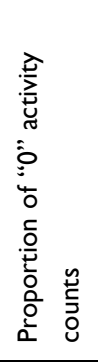 & 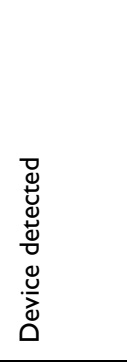 \\
\hline & 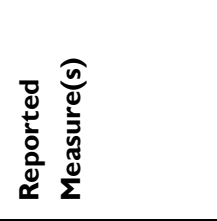 & 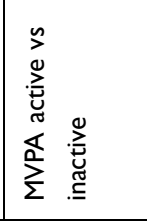 & 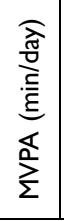 & 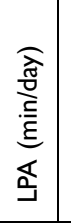 & 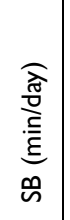 & 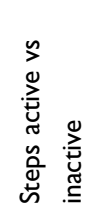 & 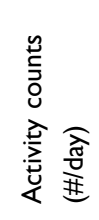 & 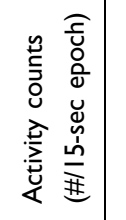 & 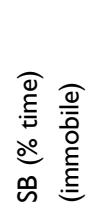 & 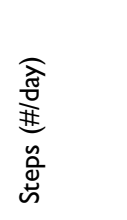 \\
\hline \multirow{2}{*}{ 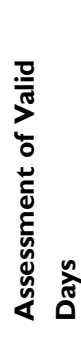 } & 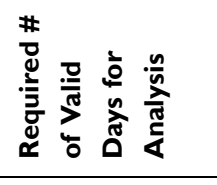 & 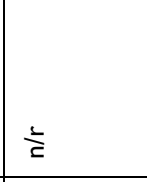 & $\grave{c}$ & & & $\grave{c}$ & 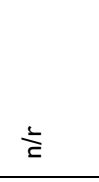 & 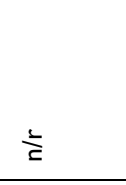 & & $\sigma$ \\
\hline & 흘 & \pm & 으 & & & 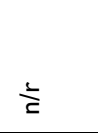 & $\grave{1}$ & 으 & & 으 \\
\hline \multirow{5}{*}{ 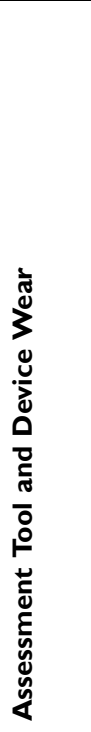 } & 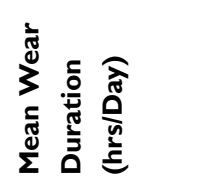 & $\simeq$ & 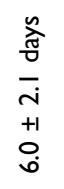 & & & $\grave{c}$ & $\grave{c}$ & 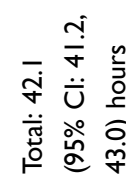 & & 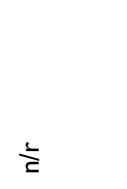 \\
\hline & 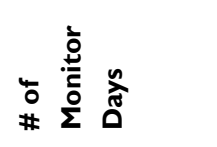 & 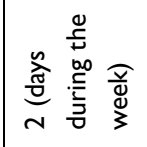 & $\wedge$ & & & $\lambda$ & $r$ & $m$ & & $\wedge$ \\
\hline & ذँ & 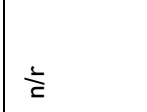 & $\stackrel{\hat{\bar{I}}}{2}$ & & & 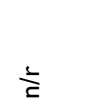 & 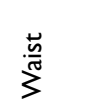 & 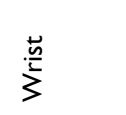 & & 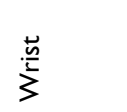 \\
\hline & 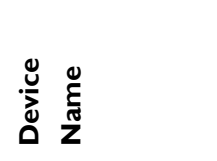 & 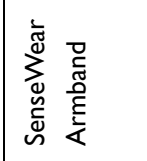 & 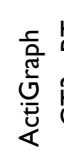 & & & 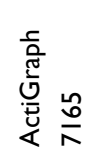 & 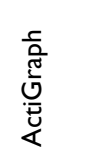 & 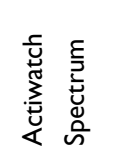 & & 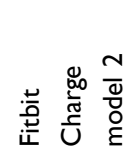 \\
\hline & « to & $\varangle$ & $\ll$ & & & $\varangle$ & $\varangle$ & $\varangle$ & & $\ll$ \\
\hline 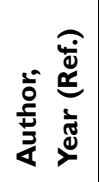 & & 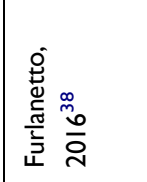 & 造 & & & $\begin{array}{l}\frac{0}{0} \\
\frac{0}{2} \\
\overline{\bar{T}} \\
\text { I }\end{array}$ & 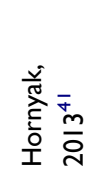 & 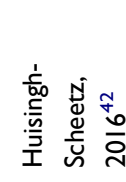 & & 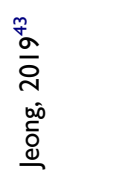 \\
\hline
\end{tabular}




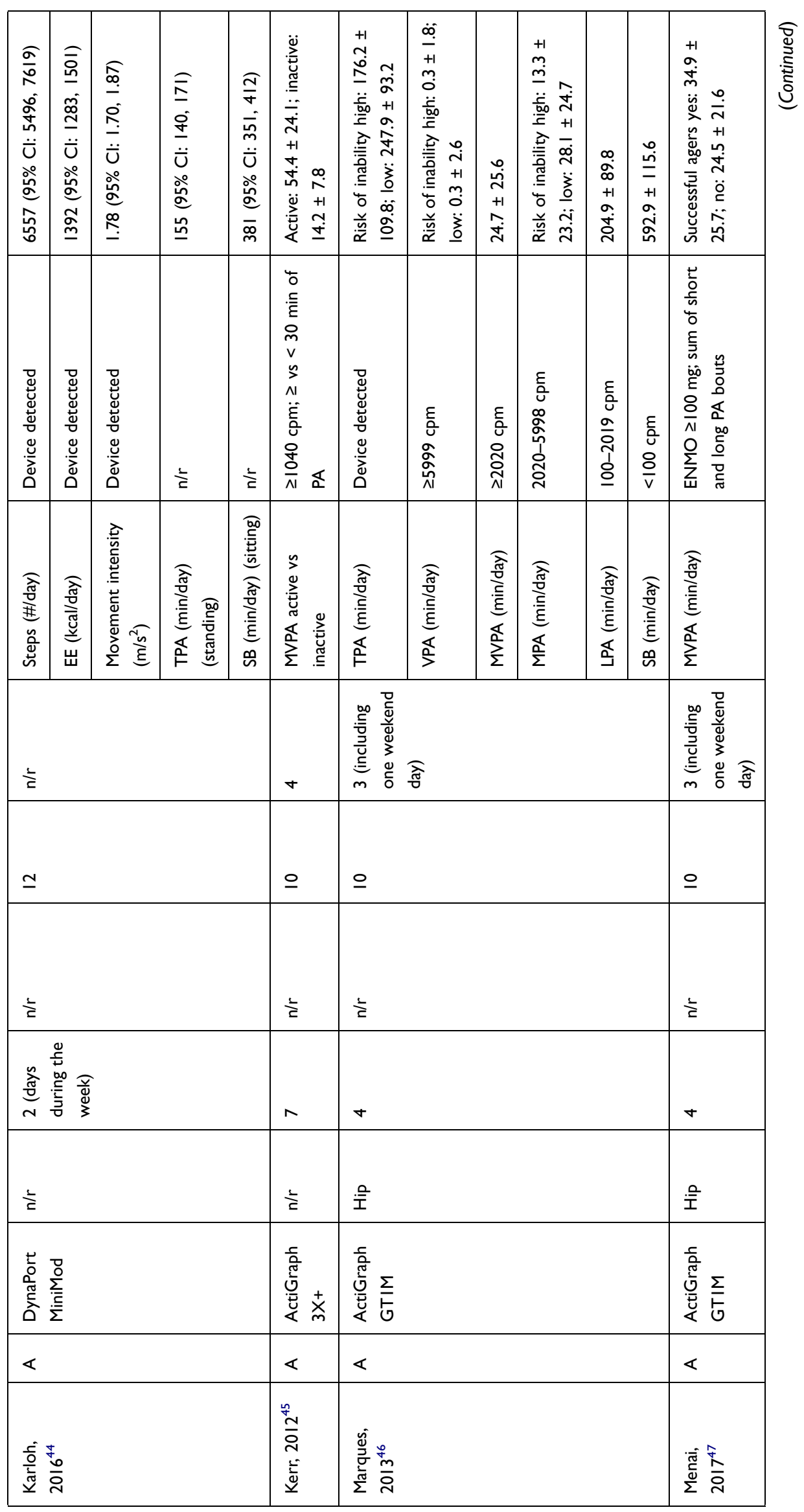




\begin{tabular}{|c|c|c|c|c|c|c|c|c|c|}
\hline \multirow{3}{*}{ 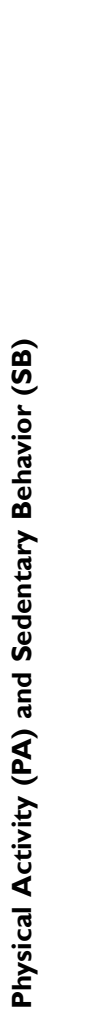 } & ڤัँ & 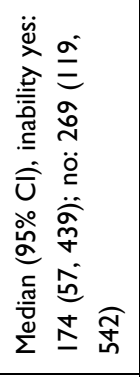 & 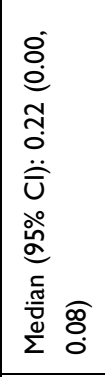 & 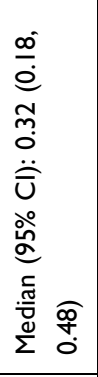 & 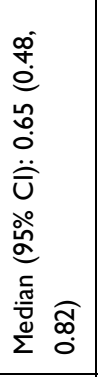 & 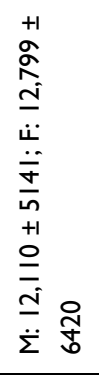 & 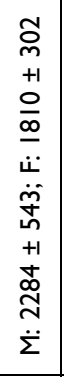 & $\begin{array}{l}\stackrel{0}{\tilde{m}} \\
\stackrel{\rho}{0} \\
+1 \\
\infty \\
\tilde{m} \\
\tilde{m}\end{array}$ & $\begin{array}{l}\stackrel{\omega}{\sim} \\
\underset{\sim}{+} \\
+1 \\
\omega \\
\infty \\
\sim\end{array}$ \\
\hline & & 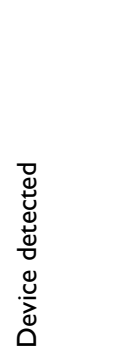 & $\begin{array}{l}\varepsilon \\
\frac{\varepsilon}{0} \\
\frac{2}{\alpha} \\
\frac{\alpha}{\wedge 1}\end{array}$ & $\begin{array}{l}\frac{\varepsilon}{0} \\
\frac{\overline{0}}{\alpha} \\
\frac{1}{0}\end{array}$ & $\begin{array}{l}\frac{E}{0} \\
\frac{8}{v 1} \\
\frac{8}{v 1}\end{array}$ & 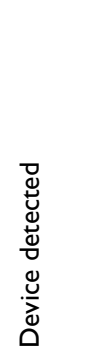 & 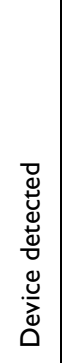 & 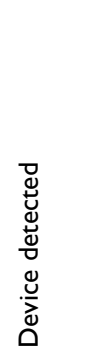 & $\begin{array}{l}\frac{0}{0} \\
\overline{0} \\
0 \\
01 \\
0 \\
\frac{0}{\Sigma}\end{array}$ \\
\hline & 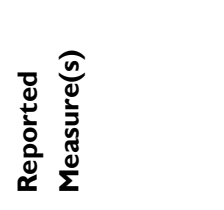 & 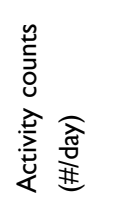 & 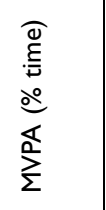 & 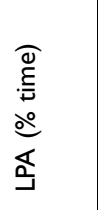 & 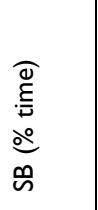 & 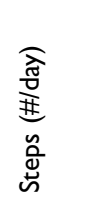 & 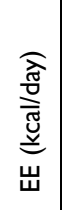 & 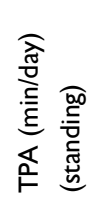 & 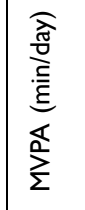 \\
\hline \multirow{2}{*}{ 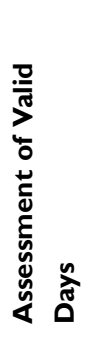 } & 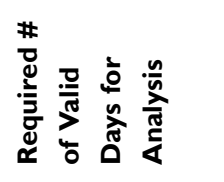 & $\nabla$ & & & & $\grave{c}$ & & - & \\
\hline & 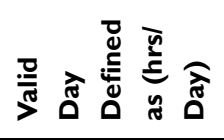 & 으 & & & & $\grave{c}$ & & $\grave{c}$ & \\
\hline \multirow{5}{*}{ 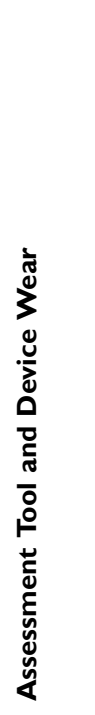 } & 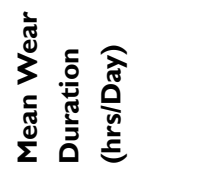 & 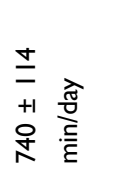 & & & & 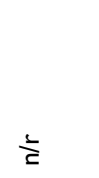 & & 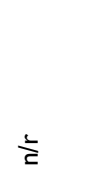 & \\
\hline & 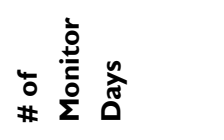 & 으 & & & & $m$ & & 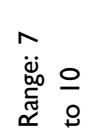 & \\
\hline & 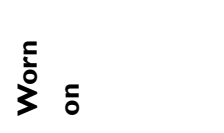 & $\stackrel{\varrho}{\underline{\underline{I}}}$ & & & & 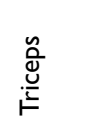 & & 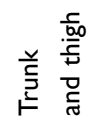 & \\
\hline & 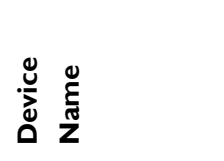 & 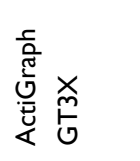 & & & & 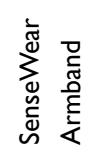 & & 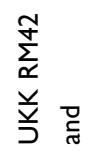 & 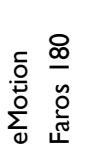 \\
\hline & $\varangle$ ò & $\varangle$ & & & & $\varangle$ & & $\varangle$ & \\
\hline 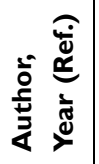 & & 遌 & & & & 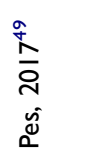 & & 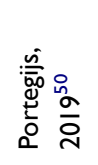 & \\
\hline
\end{tabular}




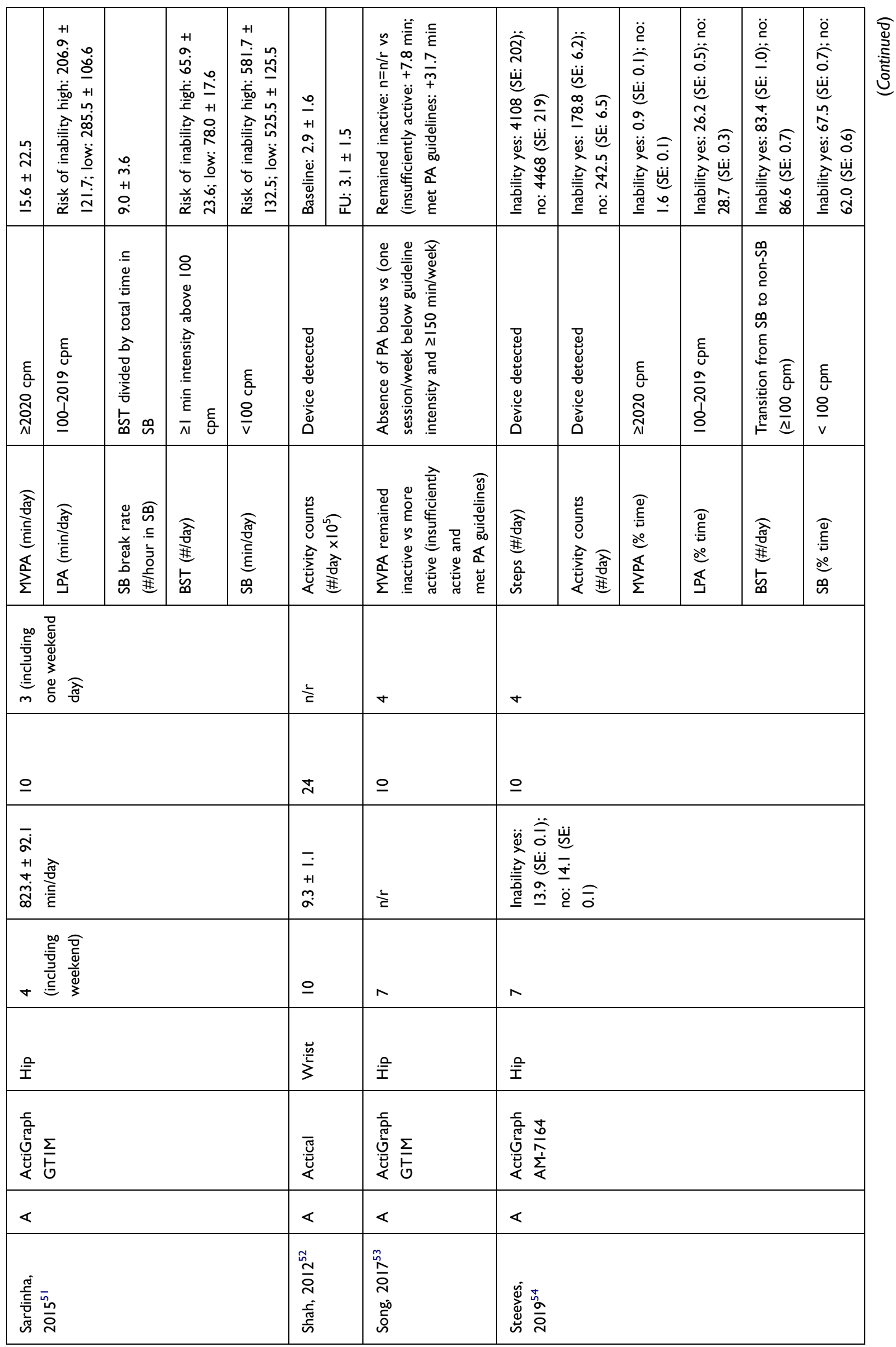




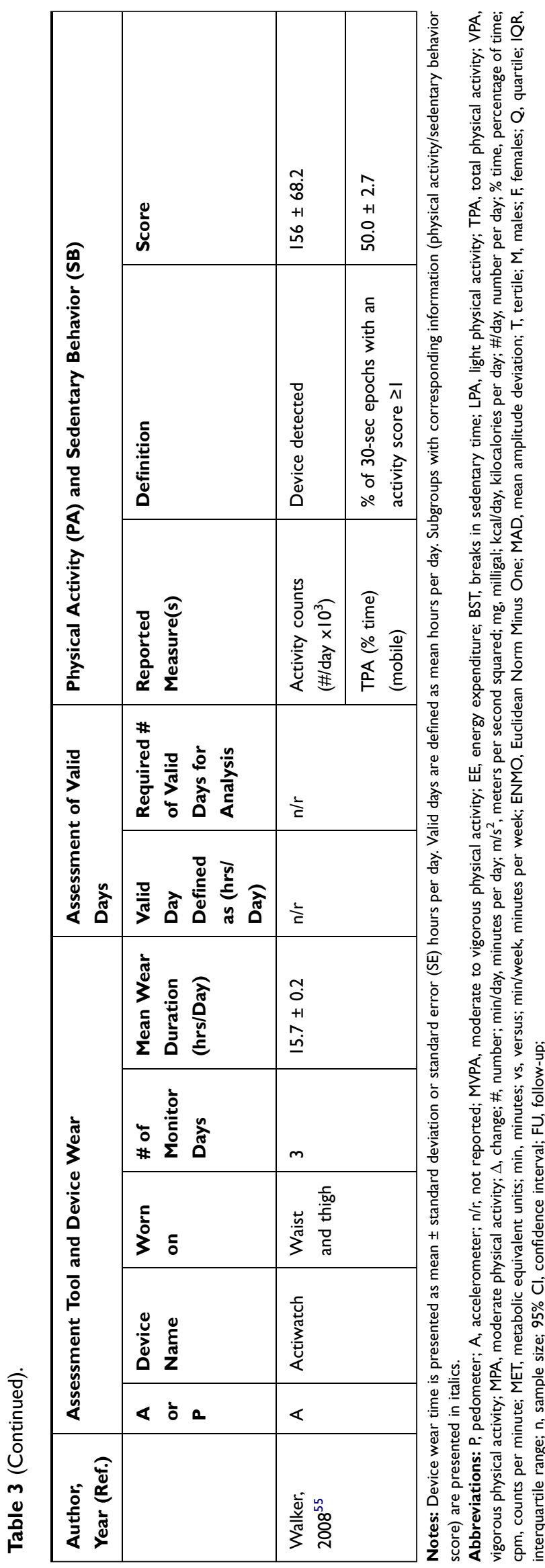

(Figure 3), and the sensitivity analyses (population selection, study design, adjustment, device type, and device wearing location) are demonstrated in Figure 4.

\section{Associations of PA and SB with ADL}

Longitudinal associations between PA/SB measures and ADL were studied in four articles; ${ }^{27,31,36,53}$ all associations were significant and effect directions showed that higher PA and lower SB were consistently associated with better ADL: lower MVPA and EE, and higher SB at baseline, were associated with an increased likelihood to become dependent in ADL after two years in community-dwelling older males, ${ }^{31}$ higher baseline activity counts was associated with a lower hazard of ADL dependence after 3.4 years in a general community-dwelling older adult population, ${ }^{53}$ engaging in approximately one-hour MVPA was associated with a lower risk of becoming dependent in ADL after four years in an osteoarthritis population, ${ }^{36}$ and a bidirectional association was identified between number of steps and ADL (a higher average number of steps was associated with better ADL from baseline and, additionally, worsened ADL from baseline was associated with a lower average number of steps) over five years in an osteoarthritis population. ${ }^{27}$ These findings were supported by cross-sectional associations, which demonstrated that higher PA and lower SB were associated with better ADL; furthermore, three articles ${ }^{28,51,55}$ studied ADL as independent and PA/SB as dependent variable, showing that limited ability to complete ADL was associated with lower PA and higher SB (Table 6; Figure 2A). The median [interquartile range] standardized regression coefficient $(\beta)$ for all articles reporting associations between PA/SB measures and ADL was 0.145 [0.072, 0.280] (Figure 3A).

\section{Associations of PA and SB with IADL}

Three articles studied longitudinal associations between PA/SB measures and IADL, ${ }^{31,34,54}$ which were all significant and had a positive effect direction: community-dwelling older male adults with lower MVPA and EE, and higher SB at baseline were more likely to become dependent in IADL after two years ${ }^{31}$ and in two articles including older adults from the Osteoarthritis Initiative (OAI), after two years follow-up, higher MVPA and LPA at baseline $^{34}$ and increasing MVPA from baseline ${ }^{54}$ were associated with a lower hazard for the development and progression of IADL dependence ${ }^{34}$ and improved IADL, ${ }^{54}$ respectively. Cross-sectional associations were in line with these results, showing that $\mathrm{PA} / \mathrm{SB}$ measures were 


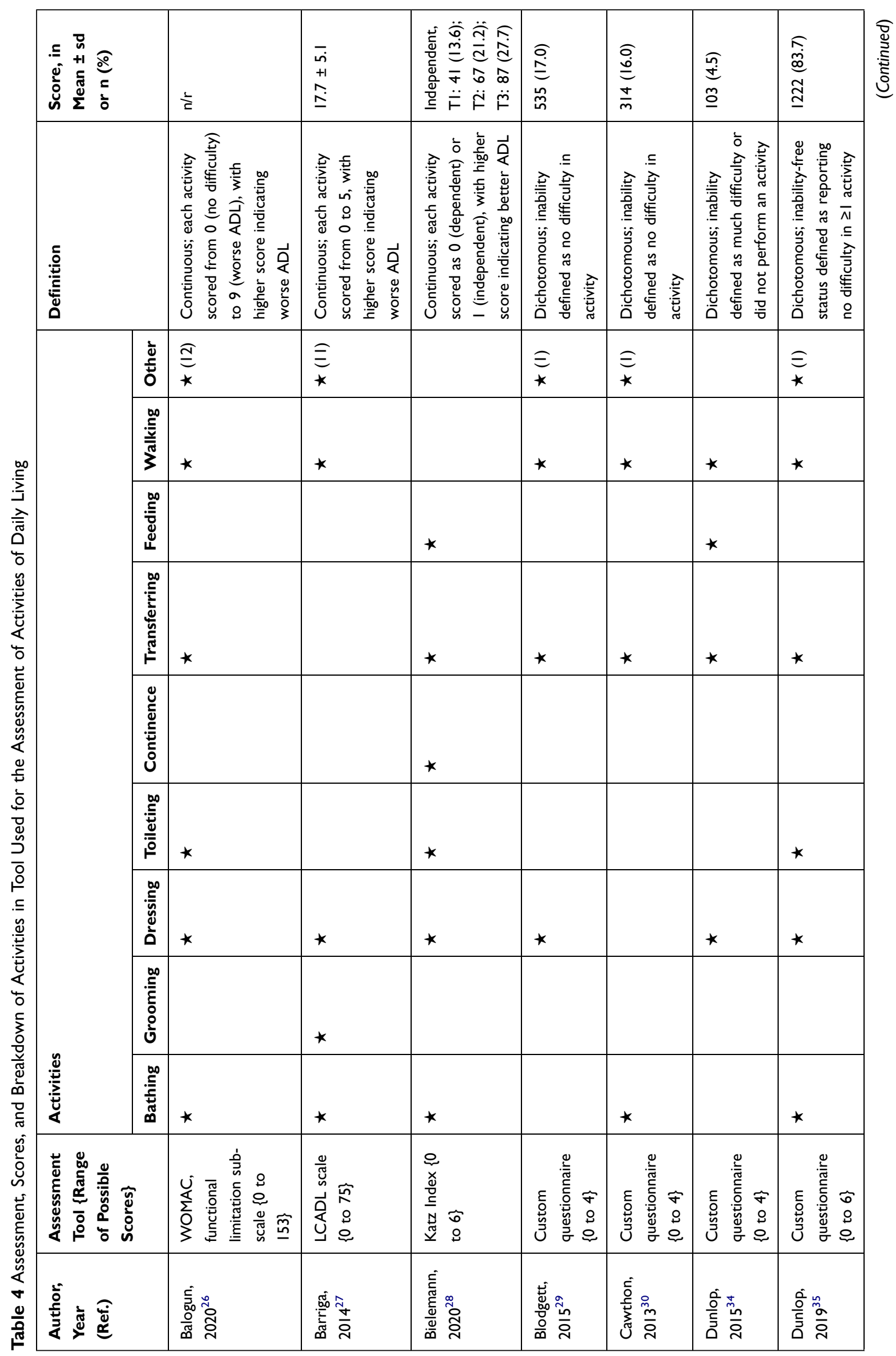




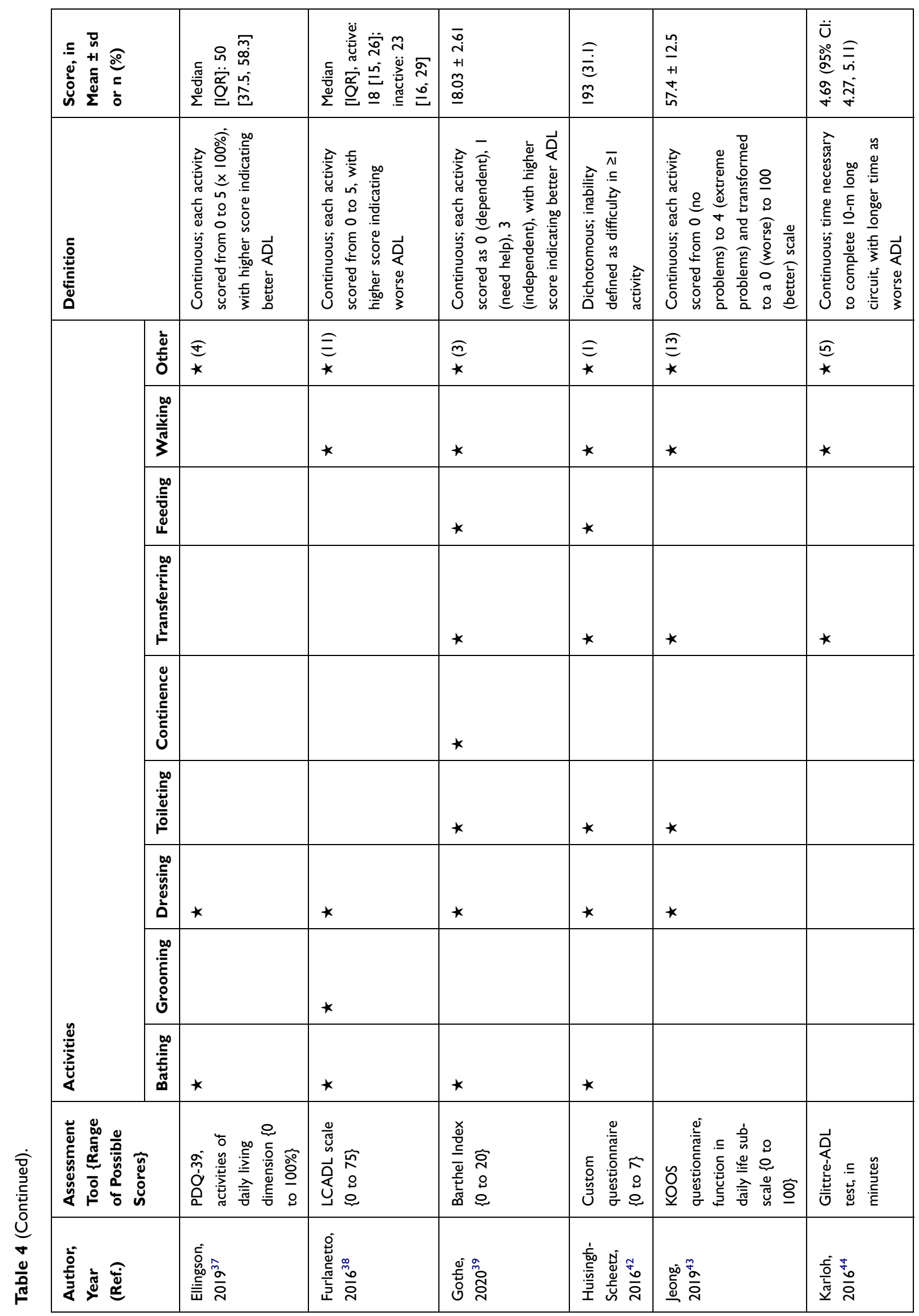




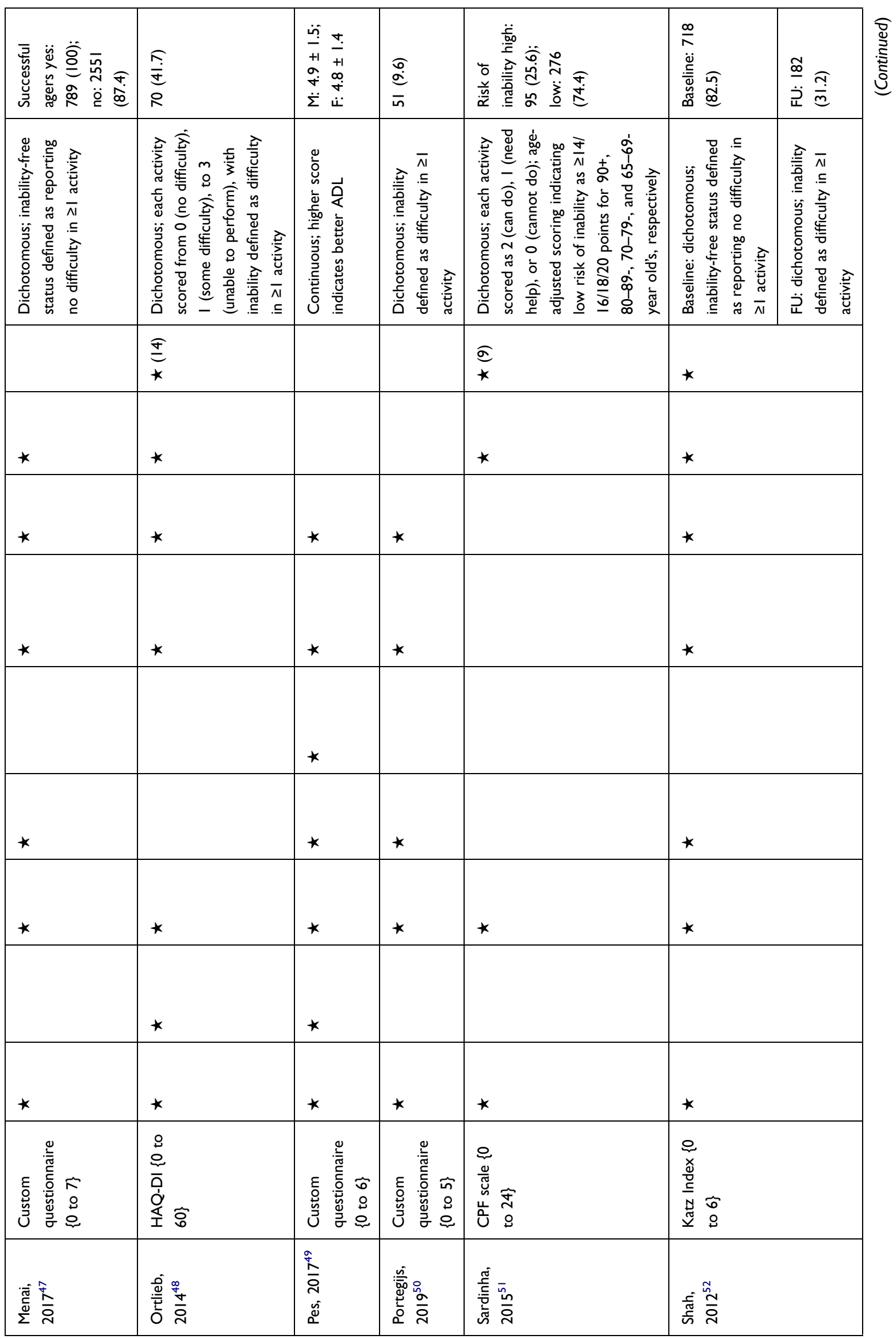




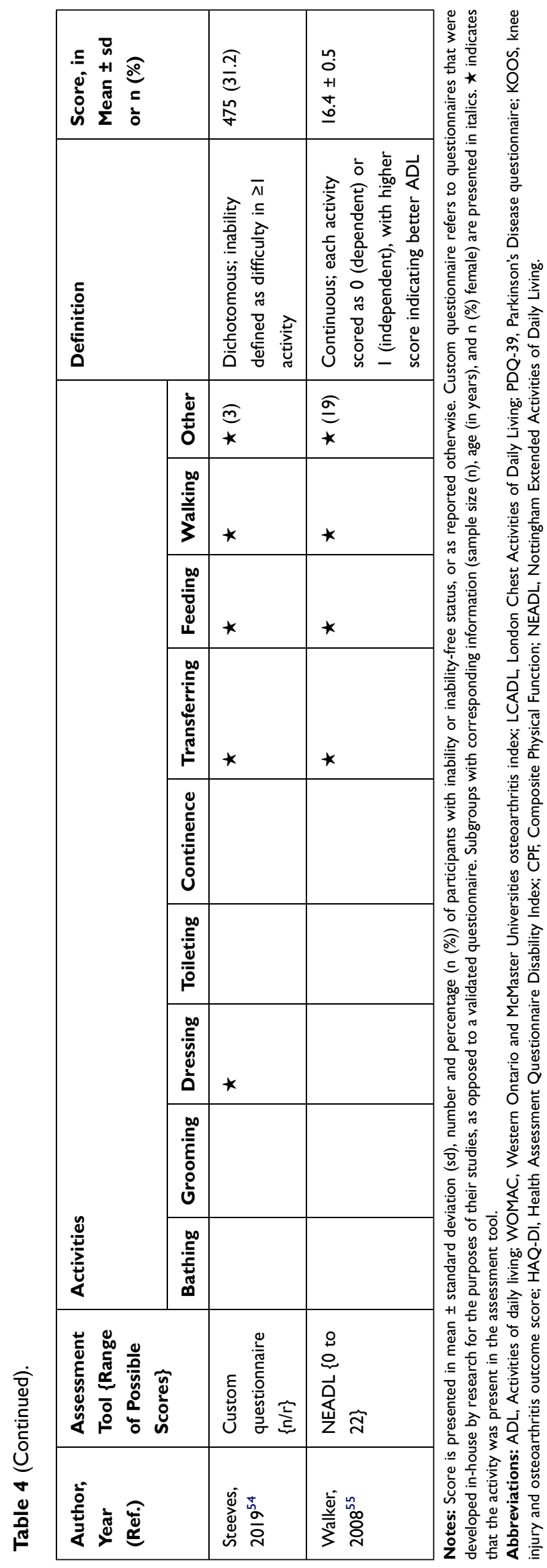

positively associated with IADL. Three studies investigated the cross-sectional association between measures of $\mathrm{PA} / \mathrm{SB}$ and IADL with IADL as independent variable and $\mathrm{PA} / \mathrm{SB}$ as dependent variable, showing that experiencing difficulty in IADL was associated with lower levels of PA (Table 6; Figure 2B). The median [interquartile range] standardized regression coefficient $(\beta)$ for all articles reporting associations between $\mathrm{PA} / \mathrm{SB}$ measures and IADL was 0.135 [0.093, 0.211] (Figure 3B).

\section{Sensitivity Analyses}

Sensitivity analyses demonstrated that population selection (general and disease populations) had an influence on the effect sizes of associations between PA/SB and, in particular, ADL with larger standardized regression coefficients found for disease populations (median [IQR]: $\beta=0.314$ $[0.159,0.460])$ than general populations (median $[\mathrm{IQR}]$ : $\beta=0.111[0.067,0.178]$ ) (Figure 4A). Longitudinal associations presented smaller standardized regression coefficients (median [IQR] for ADL: $\beta=0.078[0.065,0.120]$ and IADL: $\beta=0.084[0.069,0.094])$ when compared to cross-sectional associations (median [IQR] for ADL: $\beta=0.157$ [0.098, 0.301] and IADL: $\beta=0.162$ [0.113, 0.224]) (Figure 4B). For unadjusted associations larger standardized regression coefficients were found (median [IQR] for ADL: $\beta=0.316[0.304,0.462]$ and IADL: $\beta=0.170[0.144,0.176])$ in comparison to adjusted associations, especially for the relationship between $\mathrm{PA} / \mathrm{SB}$ and ADL (median [IQR] $\beta=0.112$ [0.072, 0.178]) (Figure 4C). In all studies, except for two that used a pedometer, accelerometers were used to monitor PA and SB (median $\beta$ [IQR] for ADL: 0.145 [0.076, 0.266] and for IADL: $0.135[0.093,0.211]$ ) (Figure 4D). For ADL, largest median standardized coefficient was observed when the device was located on the wrist (median [IQR] $\beta=0.187$ [0.082, 0.232 ], followed by a positioning on the hip (median [IQR] $\beta=0.114[0.064,0.157]$ ) and triceps (median [IQR] $\beta=0.078[0.059,0.277])$; whereas for IADL, device wearing location had no influence on the effect size (median $\beta$ [IQR] for hip: 0.162 [0.090, 0.204] and for triceps: 0.158 [0.106, 0.213]) (Figure 4E).

\section{Discussion}

Higher PA and lower SB at baseline and increased PA from baseline were consistently associated with maintaining or improving the ability to complete ADL and IADL from baseline in community-dwelling older adults. These longitudinal associations were supported by the more 


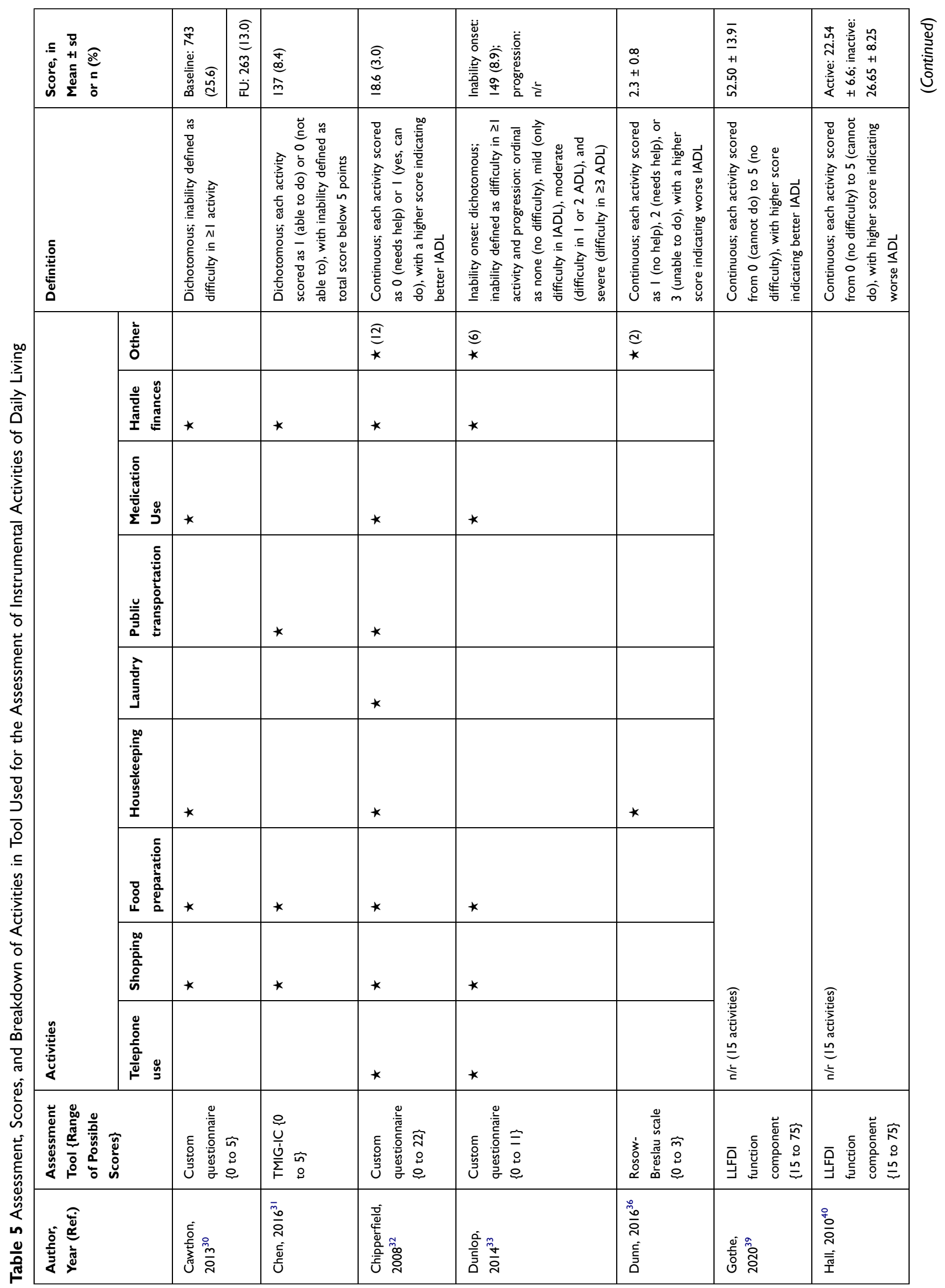




\begin{tabular}{|c|c|c|c|c|c|}
\hline 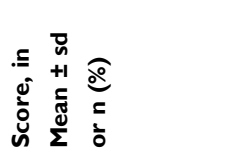 & & 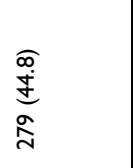 & 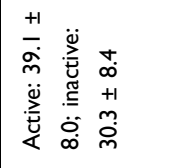 & 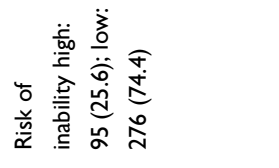 & 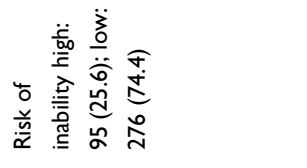 \\
\hline 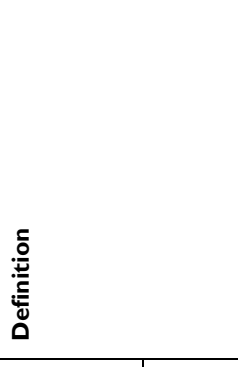 & 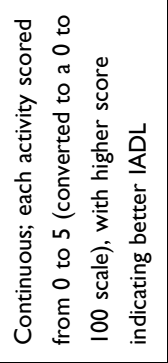 & 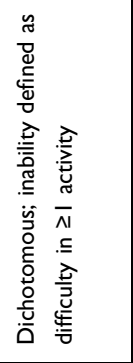 & 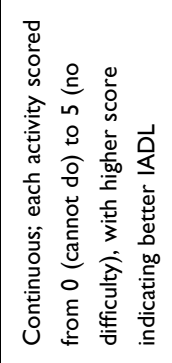 & 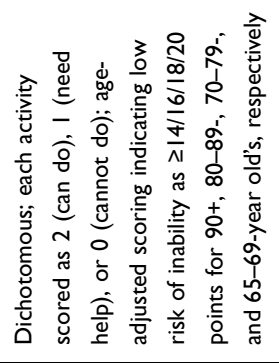 & 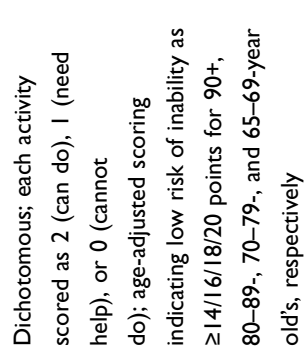 \\
\hline $\begin{array}{l}\text { 离 } \\
\text { 竎 }\end{array}$ & $\begin{array}{l}\text { తิ } \\
\star\end{array}$ & $\underset{*}{\widehat{a}}$ & & $\underset{\star}{\sigma}$ & $\underset{*}{\sigma}$ \\
\hline 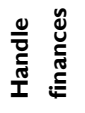 & & * & & & \\
\hline 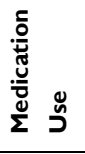 & & $*$ & & & \\
\hline 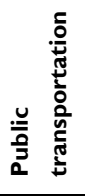 & * & & & & \\
\hline 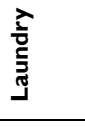 & & & & & \\
\hline 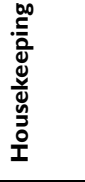 & * & * & & * & * \\
\hline r. & $*$ & * & & & \\
\hline 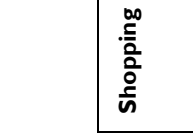 & & * & $\widehat{s}$ & * & * \\
\hline 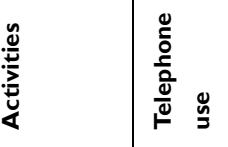 & & & 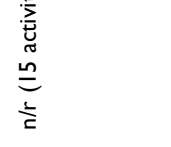 & & \\
\hline 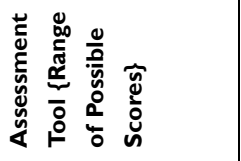 & 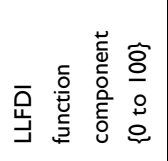 & 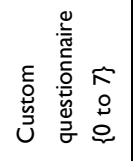 & 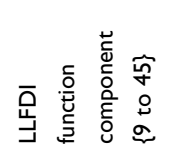 & 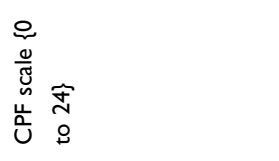 & 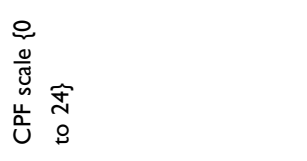 \\
\hline 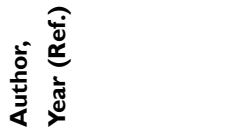 & 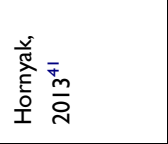 & 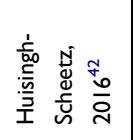 & 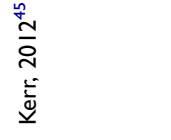 & 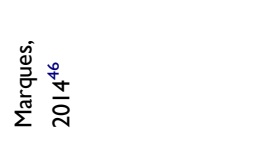 & 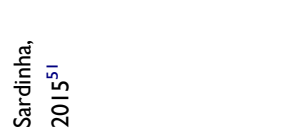 \\
\hline
\end{tabular}




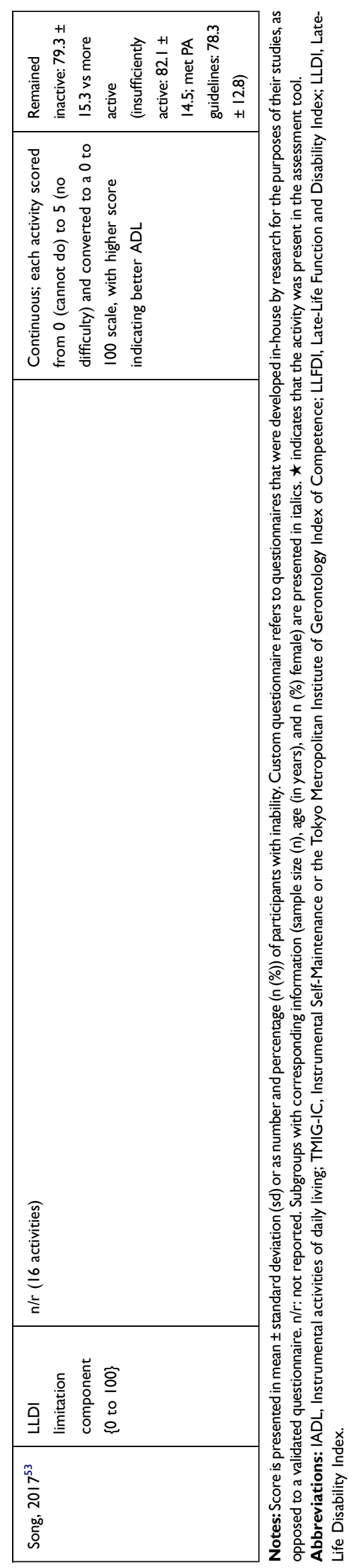

frequently reported cross-sectional studies. Effect sizes were similar for associations between $\mathrm{PA} / \mathrm{SB}$ and $\mathrm{ADL}$ or IADL; cross-sectional results yielded larger effect sizes for both ADL and IADL, and larger effect sizes were additionally found for ADL in disease populations and unadjusted analyses.

Objective measures of higher PA and lower SB showed associations with better ADL and IADL, which was in line with previous literature that purports health benefits from PA of any intensity and limited sedentary time. ${ }^{57}$ This is also in accordance with intervention studies that provide evidence of improved functional capacities in response to PA, such as coordination, muscle strength, and balance, which are essential for ADL and IADL. ${ }^{58}$

This systematic review identified similar standardized effect sizes for the association of $\mathrm{PA} / \mathrm{SB}$ measures with ADL and IADL, which was unexpected considering differences in capacities required to complete ADL and IADL. ADL primarily depends on motor functions, such as upper limb control and postural stability, that are necessary to complete the most basic forms of self-care; ${ }^{26}$ whereas, IADL additionally places a demand on cognition, particularly executive function during activities, such as grocery shopping. ${ }^{59}$ Furthermore, IADL dependence precedes ADL with the latter hence indicating greater systemlevel impairment and severe loss of autonomy. ${ }^{60}$ This is because ADL dependence is typically caused by musculoskeletal failure to where minimally demanding activities can no longer be performed. ${ }^{61}$ However, inclusion of exclusively community-dwelling older adults may have masked differences between ADL and IADL as to remain non-institutionalized requires a certain minimum ADL ability. ${ }^{62}$ While it is likely that the ability to complete $\mathrm{ADL}$ and IADL plays a role in determining to what extent someone can engage in PA, it is important to acknowledge that having the capacity to perform these activities does not ensure that the capacity is actually used to partake in PA. ${ }^{63}$

Population selection revealed dissimilarity in the effect sizes for disease versus general populations, showing that associations were dependent on the population studied, which can be explained by the pathophysiological backing regarding the effect of disease on the engagement in PA. Chronic diseases, such as COPD and osteoarthritis (commonly studied populations within this systematic review), may modify the effect that PA has on ADL because engaging in PA may be more critical for physical functioning in the presence of disease-induced impairments, such as 
A Activities of Daily Living

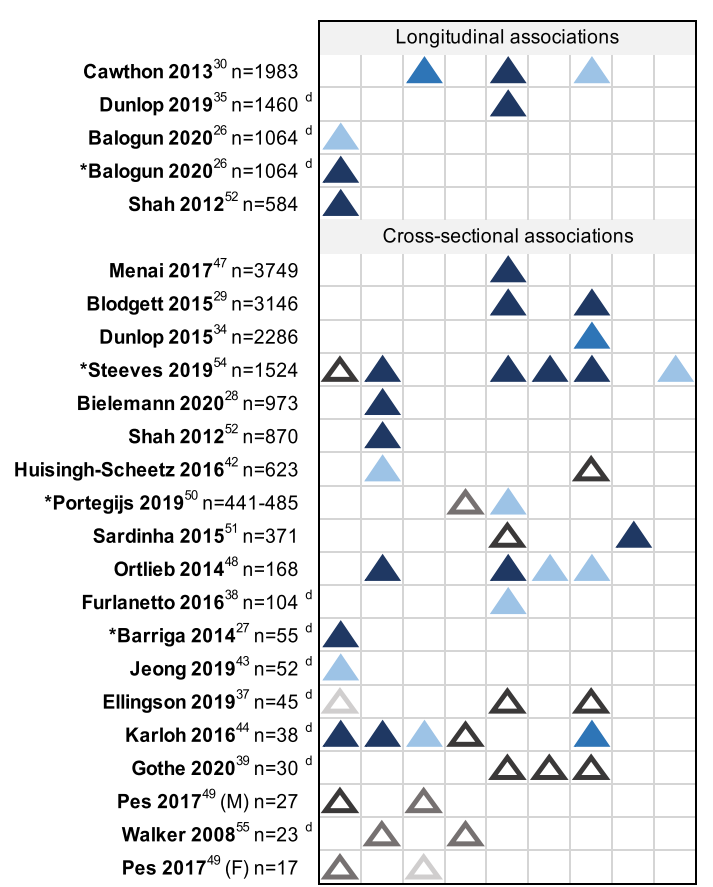

B

Instrumental Activities of Daily Living

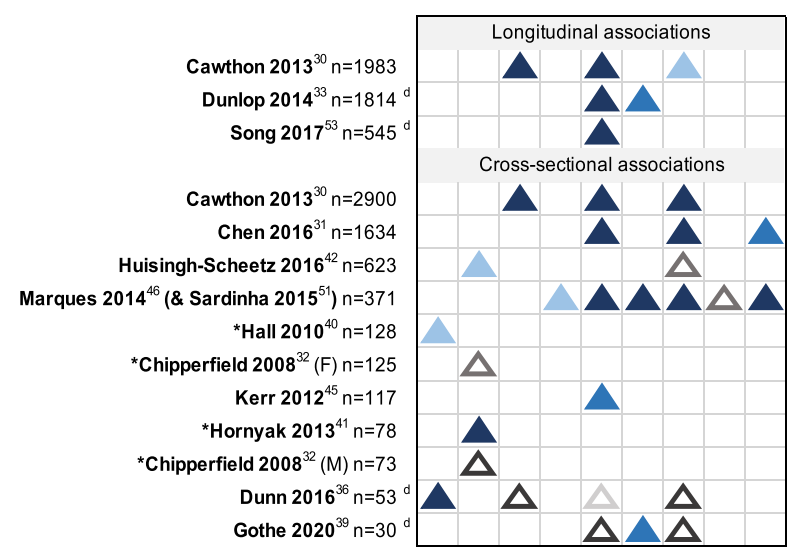

Figure 2 Effect direction heat map visualizing associations of objectively measured physical activity and sedentary behavior with (A) activities of daily living and (B) instrumental activities of daily living based on p-values, ordered by sample size, and stratified by study design (cross-sectional and longitudinal). \pm indicate positive/negative effect direction (higher PA and lower SB are associated with better (+) or worse (-) activities of daily living (ADL) or instrumental activities of daily living (IADL). PA/SB measures: Counts=activity counts, $E E=$ energy expenditure, TPA=total physical activity, MVPA=moderate to vigorous physical activity, LPA=light physical activity, $\mathrm{SB}=$ sedentary behavior, break rate=number of breaks per sedentary hour, BST=breaks in sedentary time. $\boldsymbol{\Delta} / \boldsymbol{\nabla}$ (dark blue): $\mathrm{p}<0.00 \mathrm{I}, \boldsymbol{\Delta} / \boldsymbol{\nabla}$ (blue): $0.00 \mathrm{I} \leq \mathrm{p}<0.0 \mathrm{I}, \boldsymbol{\Delta} / \boldsymbol{\nabla}$ (light blue): $0.01 \leq p<0.05, \Delta / \nabla$ (light grey): $0.05 \leq p<0.1, \Delta / \nabla$ (grey): $0.1 \leq p<0.25, \Delta / \nabla$ (dark grey): $p \geq 0.25$. *activities of daily living or instrumental activities of daily living as independent variables and PA/SB as dependent variable. ${ }^{\mathrm{d} D}$ Disease population.

Abbreviations: M, Males; F, Females.

breathlessness and stiffness, and inversely, SB may be more detrimental in the presence of disease. Stratification by study design showed that there were smaller effect sizes for longitudinal studies when compared to cross-sectional studies, which may suggest that while baseline PA and SB are determinants of baseline and future ability to perform ADL and IADL, changes in ADL and IADL in short periods of time may be more affected by other factors involved in health status. Larger effect sizes found for unadjusted associations in comparison to adjusted associations strengthen the importance of our adjustment hierarchy, which was applied to prevent inflation by confounders, such as age and sex, that may mask the actual relationship of $\mathrm{PA} / \mathrm{SB}$ with $\mathrm{ADL}$ and IADL.

$\mathrm{PA}$ and $\mathrm{SB}$ are, as highlighted in this systematic review, associated with the ability to independently accomplish ADL and IADL. Enhancing PA and reducing the time spent sedentarily are therefore promising strategies to maintain functional independence. With increasing age, however, multimorbidity and cognitive impairment are more prominent and threaten healthy aging. ${ }^{64}$ It may therefore be that the inability to perform ADL and IADL influences PA engagement and involves higher levels of SB, resulting in an overall more inactive lifestyle. Conversely, an active lifestyle could protect older adults from a loss of functional independence, which is implicated by our longitudinal findings. To disentangle this reverse causation, future randomized controlled trials are advised to inform public health strategies about an attainable active lifestyle for older adults based on their functional capability.

Population aging is accompanied by an increase in disease burden among older adults, which threatens functioning in daily life and, therefore, underpins the clinical relevance of our findings that objectively measured PA and SB are modifiable lifestyle factors of the ability to carry out ADL and IADL. This can be used to determine the dose-response relationship of PA and SB with ADL and IADL to guide public health and clinical interventions for preventing and delaying loss of independence. 


\section{A Activities of Daily Living (ADL)}

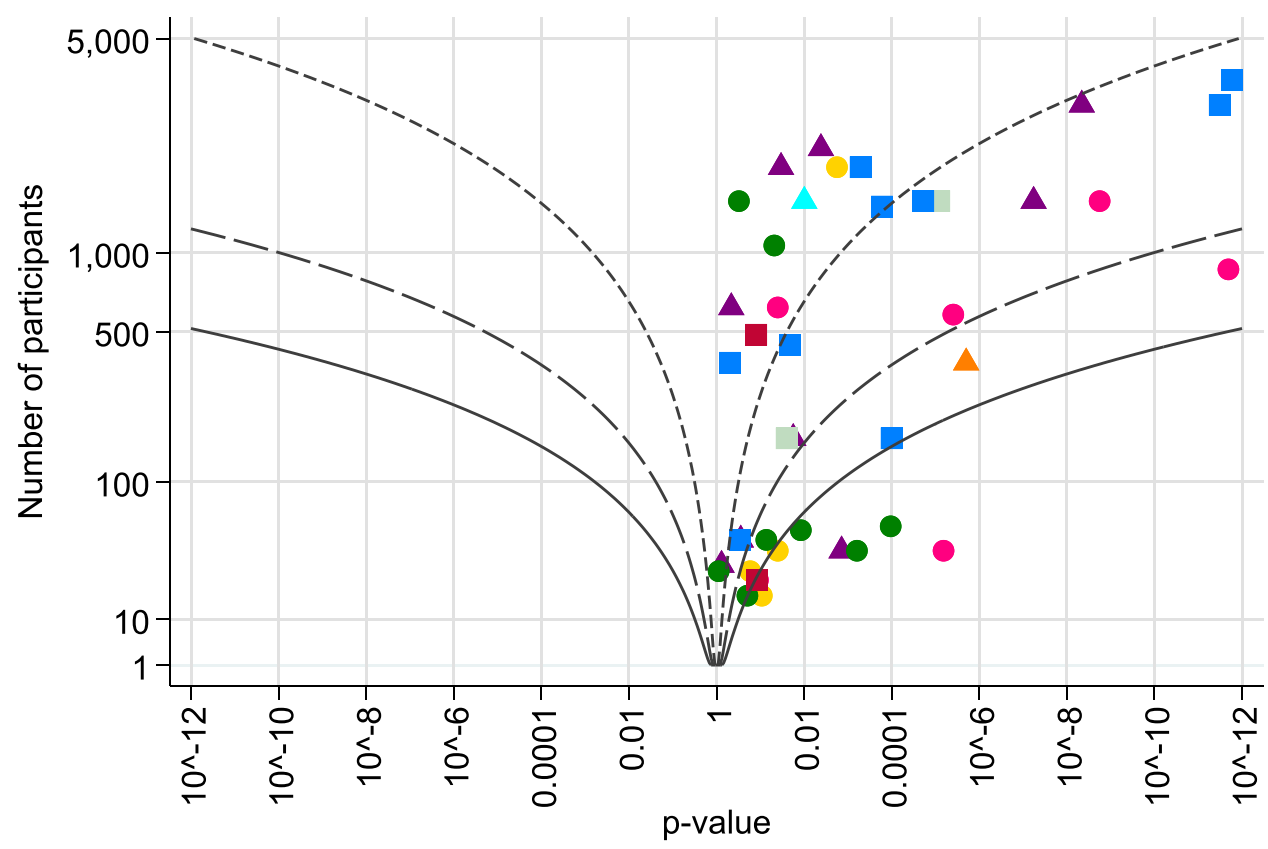

Worse ADL

Better ADL

\section{B Instrumental Activities of Daily Living (IADL)}

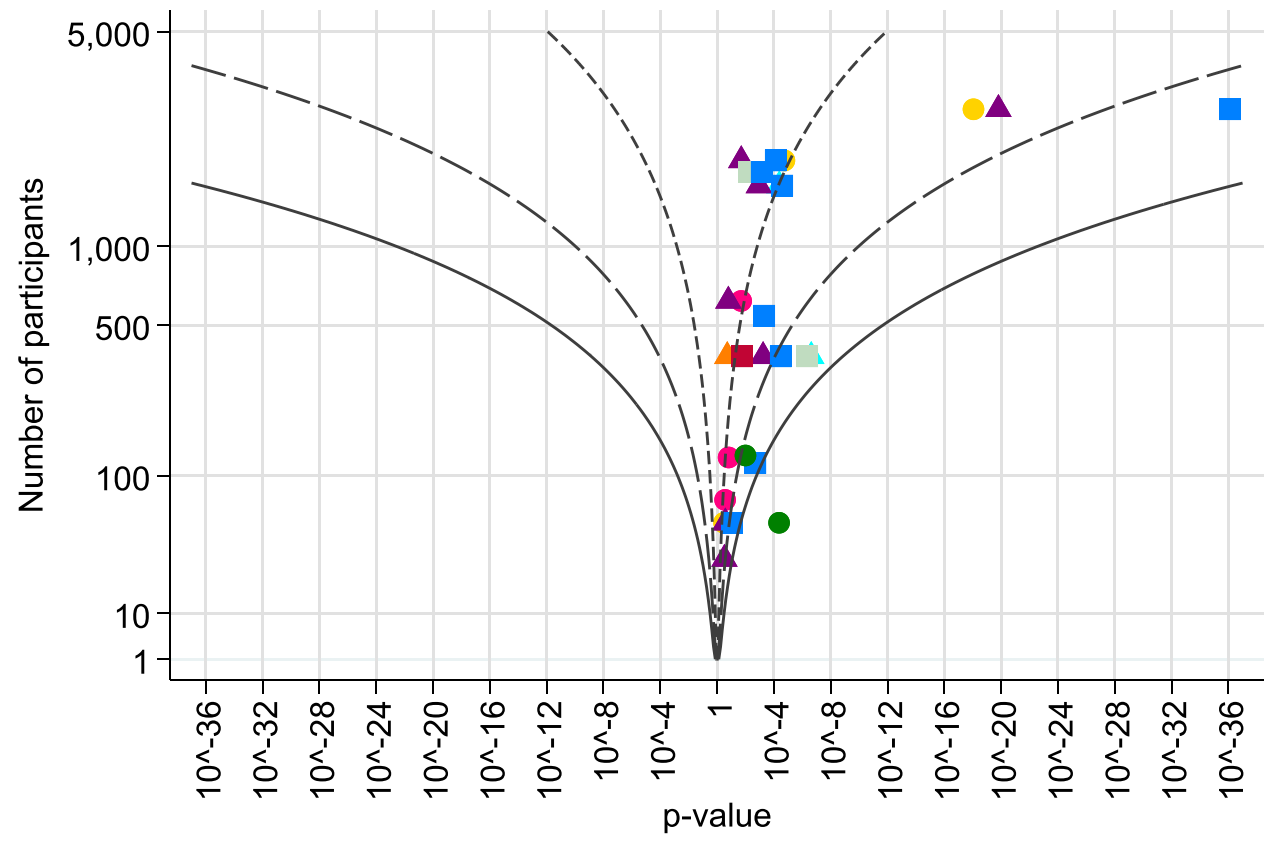

Worse IADL

Better IADL

Figure 3 Albatross plots depicting the magnitude of associations, provided as standardized regression coefficients ( $\beta$ s), of higher physical activity (PA) and lower sedentary

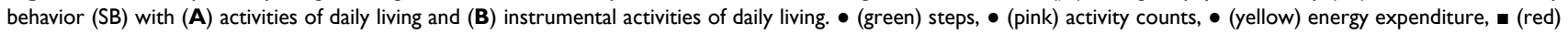
total physical activity, $\mathbf{-}$ (blue) moderate to vigorous physical activity, - (light green) light physical activity, $\boldsymbol{\Delta}$ (purple) inverse sedentary behavior, $\boldsymbol{\Delta}$ (orange) break rate (number of breaks per sedentary hour), $\boldsymbol{\Delta}$ (cyan) breaks in sedentary time. $\beta= \pm 0.10, \beta= \pm 0.20, \beta= \pm 0.30$. 


\section{A Population}

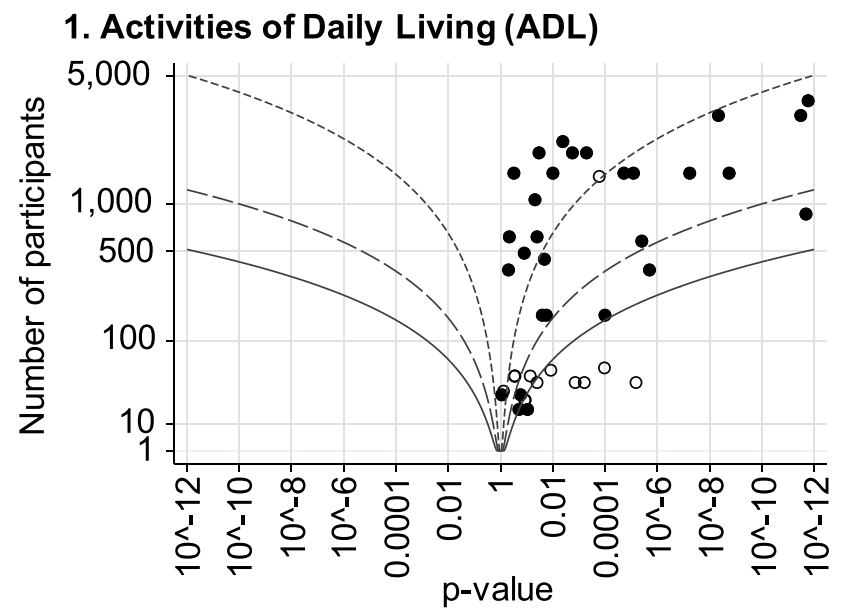

Worse ADL

\section{B Study design}

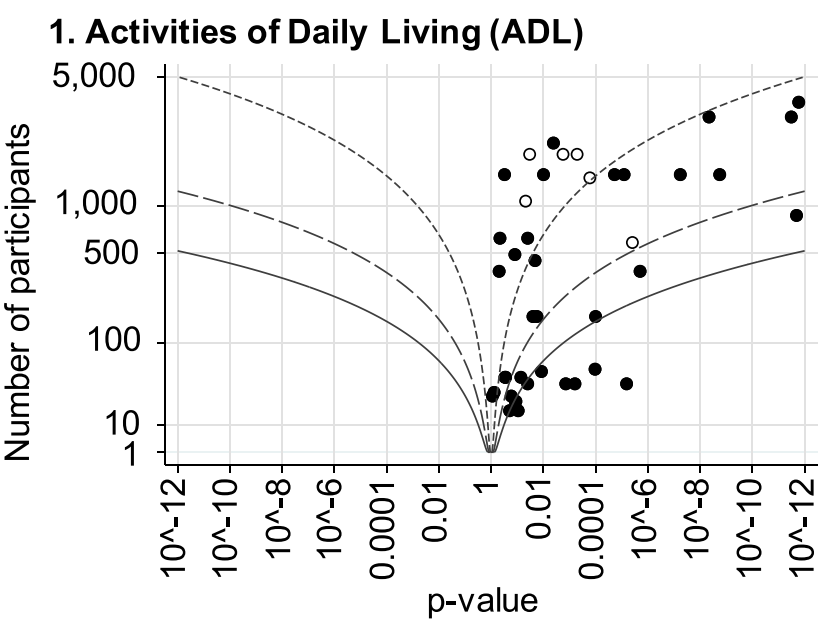
Worse ADL

Better ADL

\section{Adjustment}

\section{Activities of Daily Living (ADL)}

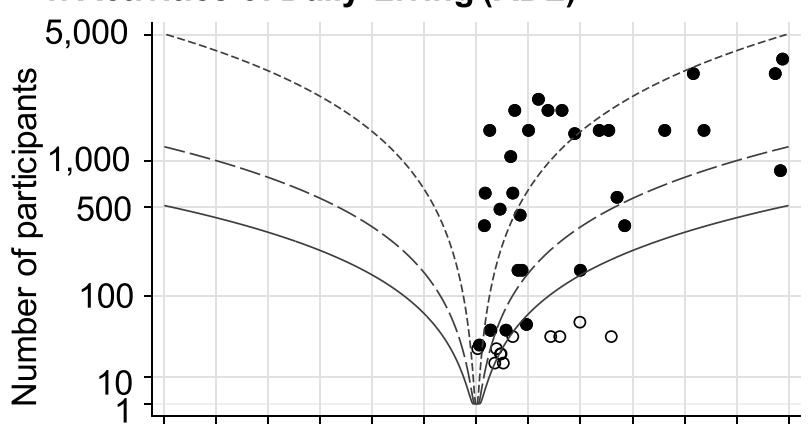

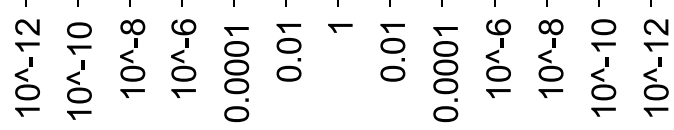
p-value

Worse ADL

Better ADL

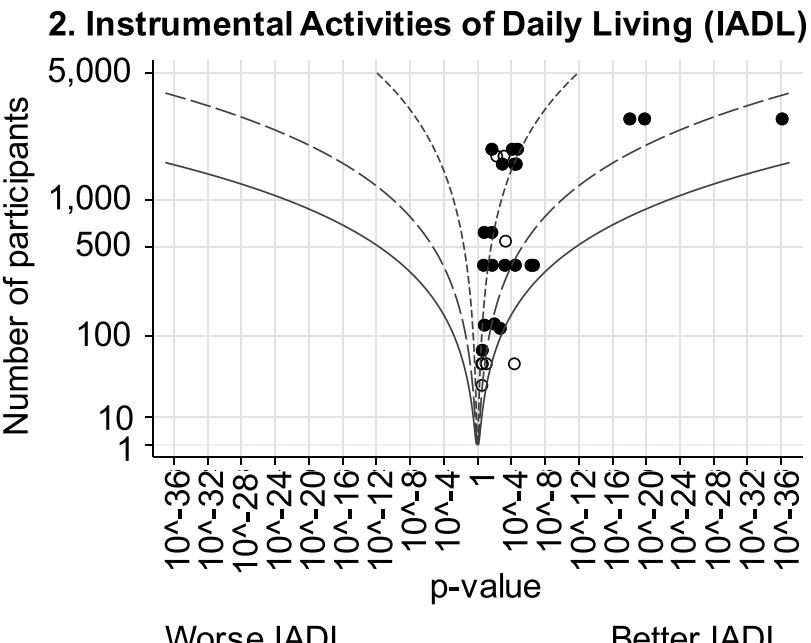

Worse IADL

Better IADL

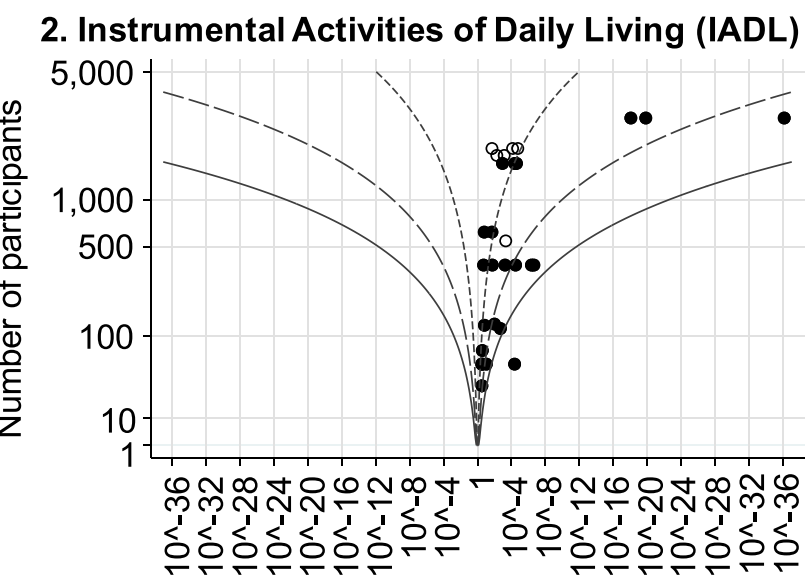

p-value

Worse IADL Better IADL

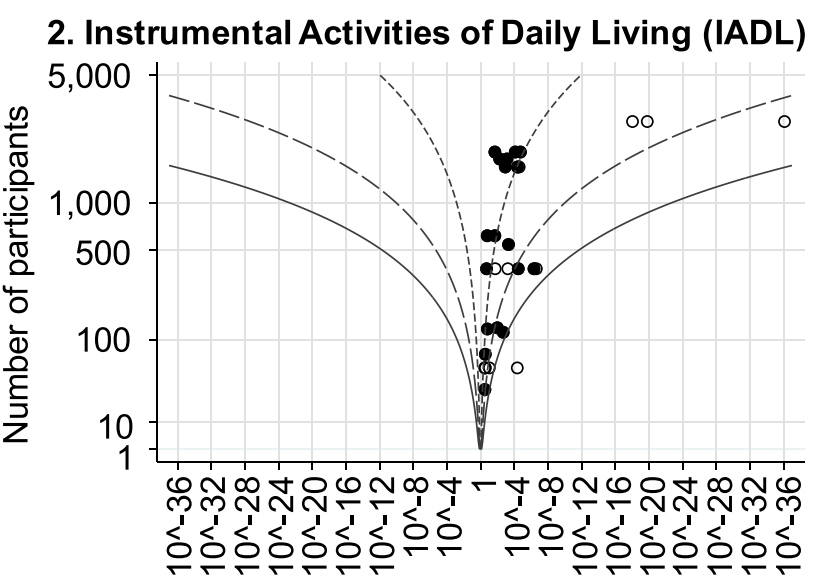

p-value

Worse IADL

Better IADL

Figure 4 Continued. 


\section{Device type}

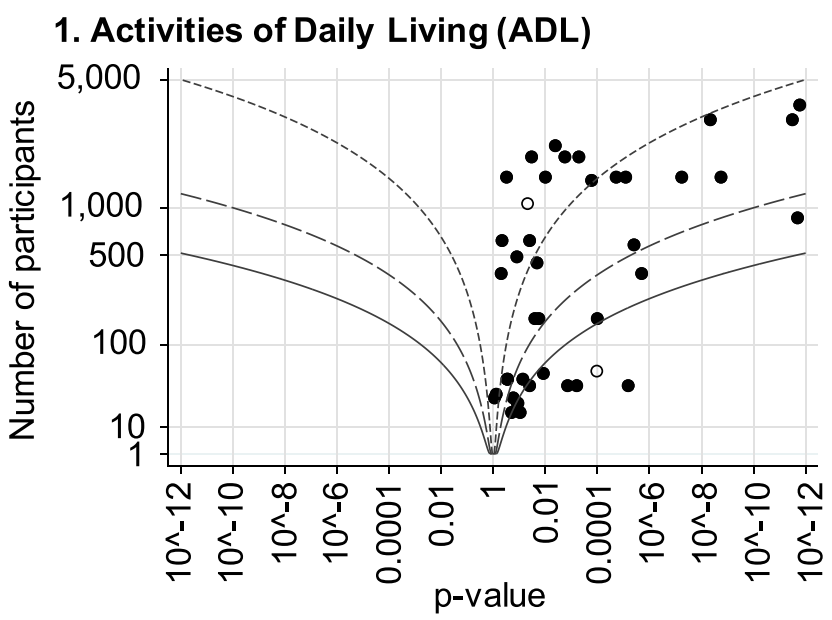

Worse ADL

Better ADL

\section{E Device wearing location}

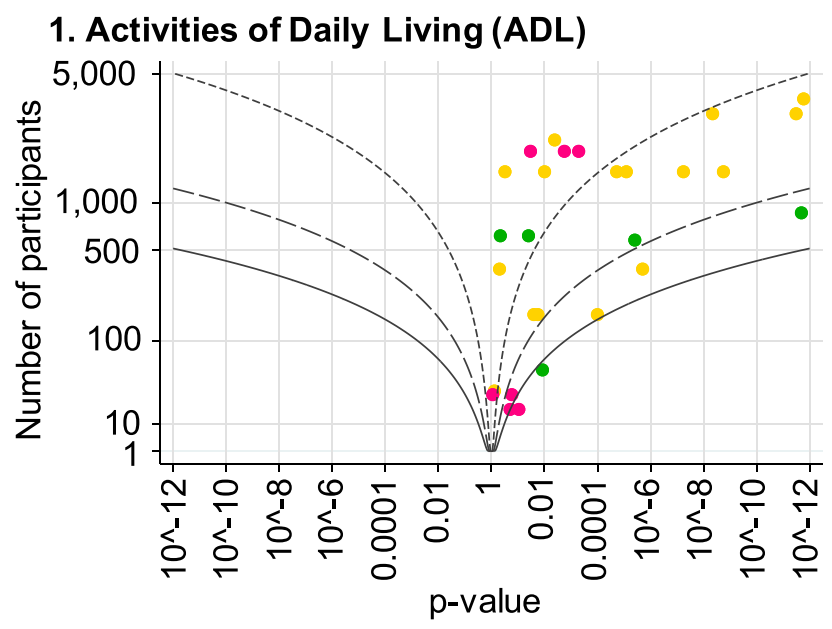

Worse ADL

Better ADL

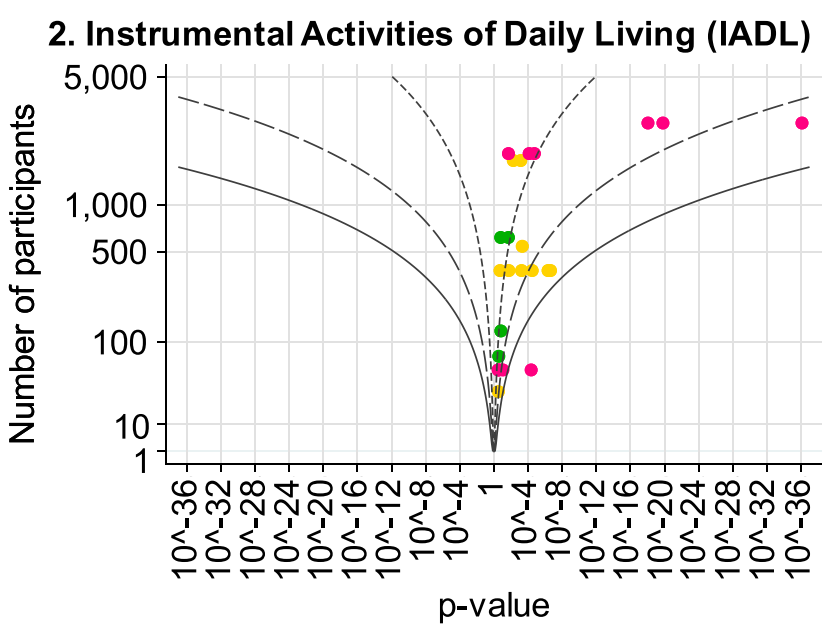

Worse IADL

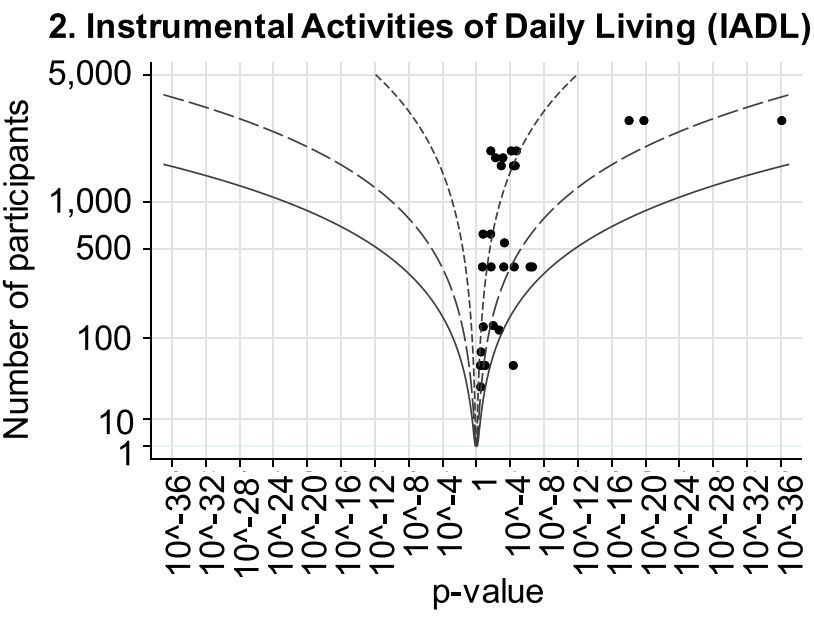

Worse IADL

Better IADL

Figure 4 Albatross plots depicting the magnitude of associations, provided as standardized regression coefficients ( $\beta$ s), of higher physical activity (PA) and lower sedentary behavior (SB) with activities of daily living (ADL) and instrumental activities of daily living (IADL), stratified by (A) population (general versus disease), (B) study design (cross-sectional versus longitudinal), (C) adjustment (adjusted versus unadjusted associations), (D) device location (accelerometer versus pedometer), and (E) device

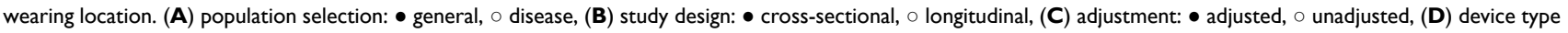
- accelerometer, $\circ$ pedometer, (E) device wearing location: $\bullet$ (green) wrist, $\bullet$ (pink) triceps, $\bullet$ (yellow) hip. $\beta= \pm 0.10, \beta= \pm 0.20, \beta= \pm 0.30$.

Considering the importance of an active lifestyle for maintaining independence, as shown in this systematic review, PA may act as a target for future intervention studies. Future studies should aim to improve standardization in the assessment of PA and SB (eg, device-wearing location, cut-off points, and assessment of ADL and IADL) to unravel the dose-response relationships of $\mathrm{PA}$ and $\mathrm{SB}$ with ADL and IADL and, ultimately, establish thresholds to prevent deterioration in the ability to complete ADL and IADL.

The inclusion of solely articles that objectively measured PA and SB is a strength of this systematic review as it eliminates bias that is involved in self-reported assessment and thus provides the most accurate insight into PA and SB and the subsequent association with ADL and IADL. As older adults regularly spend most of their time in low-intensity activities, a broad range of PA measures, including LPA, is an additional strength because this metric is often neglected due to the difficulty of measuring LPA via self-report. ${ }^{65}$ Furthermore, diverse communitydwelling older adults were included, without exclusion of specific disease groups, which allows for generalizability of our findings. Another strength is that the literature search focused on articles that were explicitly described 


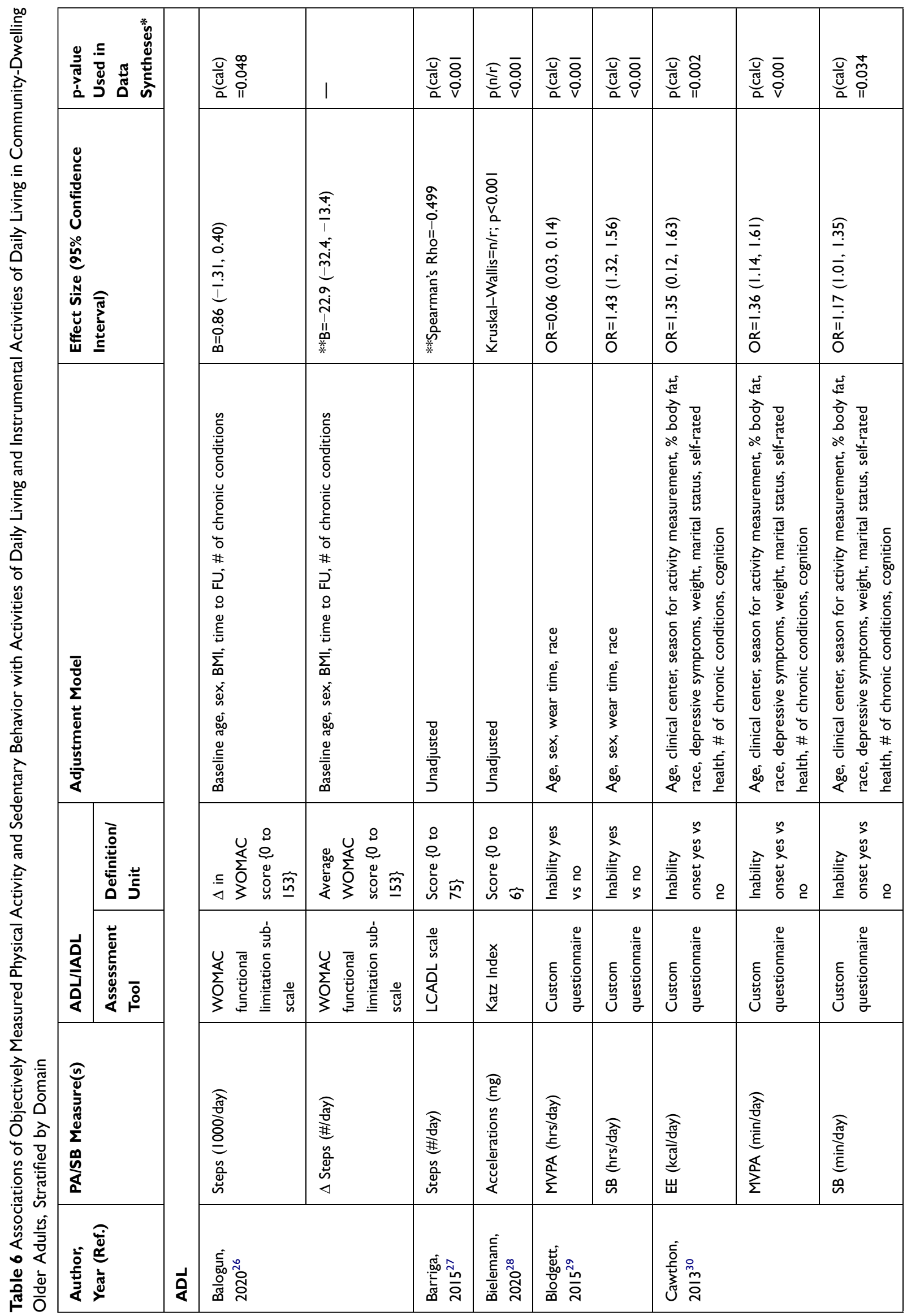




\begin{tabular}{|c|c|c|c|c|c|c|c|c|c|c|}
\hline 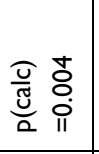 & 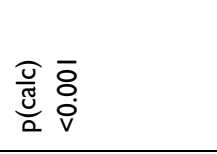 & 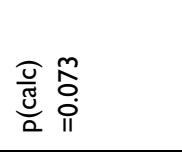 & 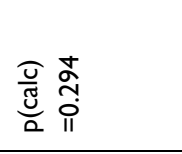 & 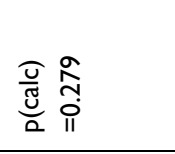 & $\begin{array}{l}\frac{c}{5} \\
\text { con } \\
\text { vi } \\
\frac{0}{0} \\
0 \\
0 \\
0\end{array}$ & 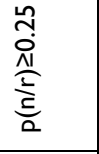 & 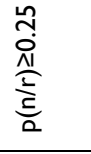 & 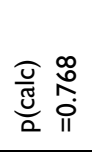 & $\begin{array}{l}\text { to } \\
0 \\
\text { II } \\
0\end{array}$ & $\begin{array}{l}\text { ț. } \\
\text { II } \\
\text { In }\end{array}$ \\
\hline 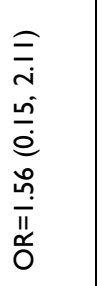 & $\begin{array}{l}\widehat{0} \\
\stackrel{0}{0} \\
0 \\
0 \\
0 \\
0 \\
0 \\
0 \\
0 \\
0 \\
0 \\
\frac{11}{\alpha}\end{array}$ & 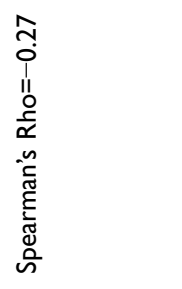 & 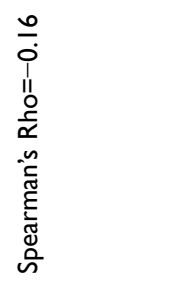 & 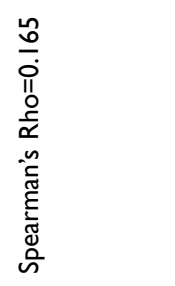 & 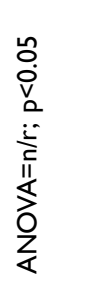 & 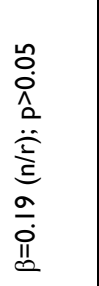 & 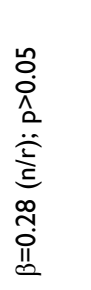 & 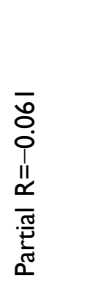 & 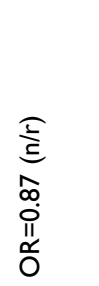 & 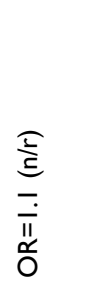 \\
\hline 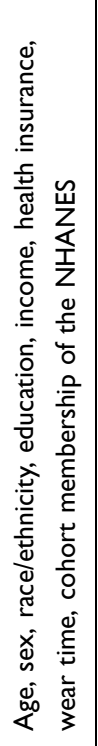 & 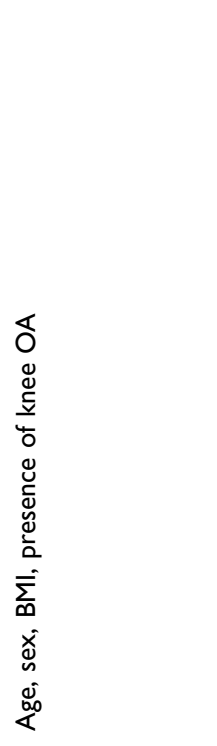 & 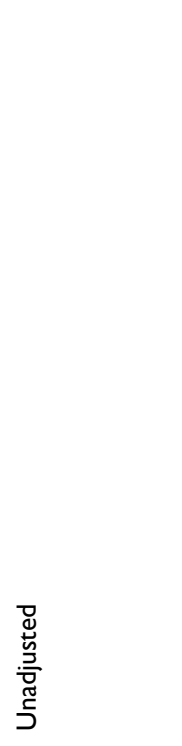 & 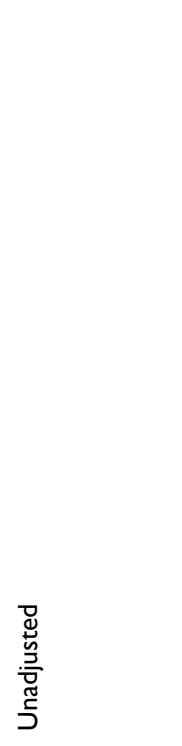 & 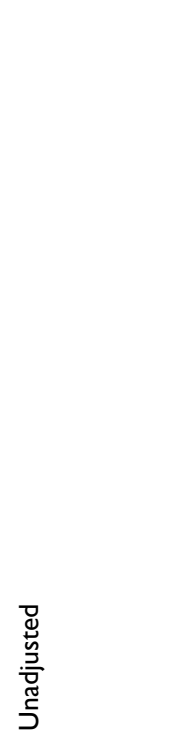 & 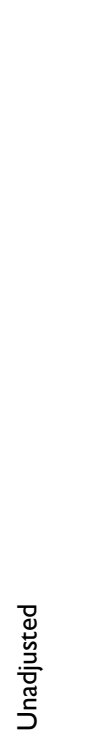 & 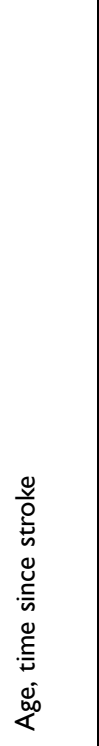 & 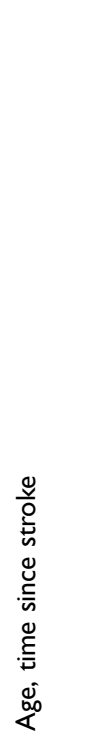 & 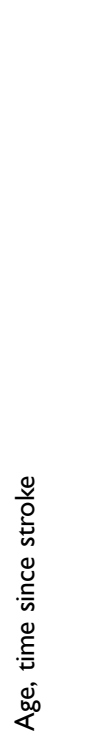 & 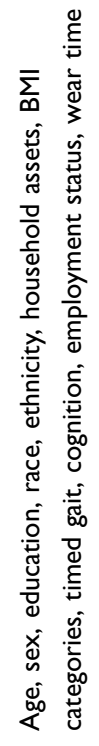 & 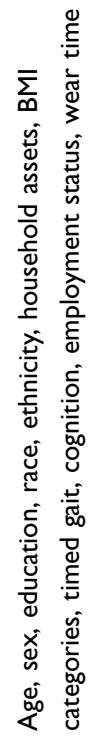 \\
\hline 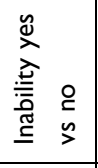 & 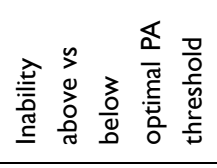 & $\begin{array}{l}\stackrel{0}{0} \\
\stackrel{0}{0} \text { o̊ } \\
\stackrel{0}{0}\end{array}$ & 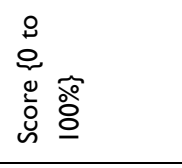 & $\begin{array}{l}\stackrel{2}{0} \\
\stackrel{0}{0} \\
\stackrel{0}{0} \\
\dot{u}\end{array}$ & $\begin{array}{l}\stackrel{8}{0} \\
\stackrel{0}{0} \\
\stackrel{0}{0} \\
\stackrel{\tilde{n}}{n}\end{array}$ & 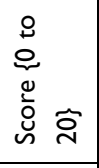 & $\begin{array}{l}\stackrel{0}{0} \\
\stackrel{0}{0} \\
\stackrel{0}{0} \\
\stackrel{i}{n}\end{array}$ & $\begin{array}{l}\stackrel{8}{0} \\
\stackrel{0}{0} \\
\stackrel{0}{0} \\
\stackrel{\sim}{n}\end{array}$ & 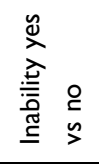 & 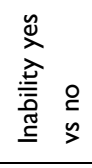 \\
\hline 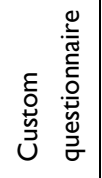 & 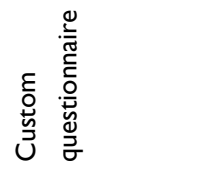 & 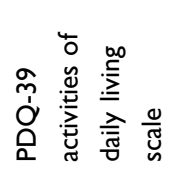 & 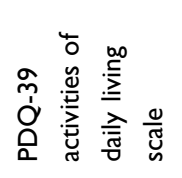 & 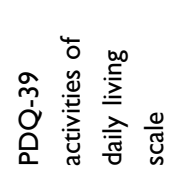 & 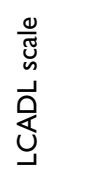 & 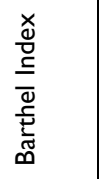 & 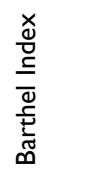 & 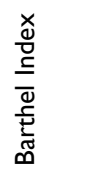 & 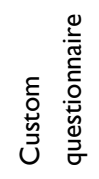 & 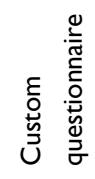 \\
\hline 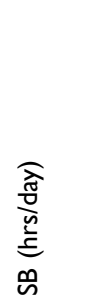 & 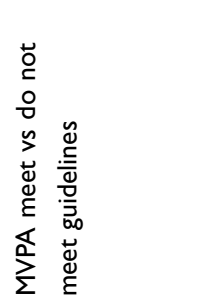 & 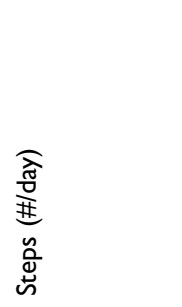 & 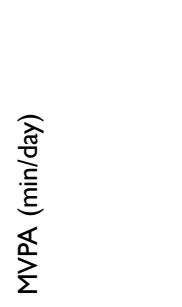 & 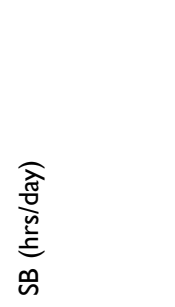 & 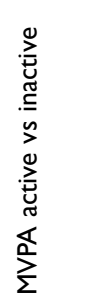 & 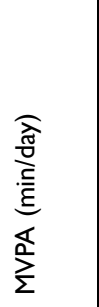 & 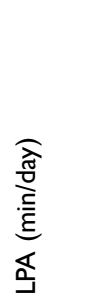 & 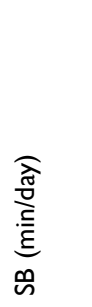 & 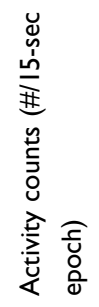 & 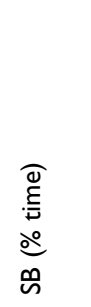 \\
\hline 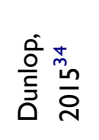 & 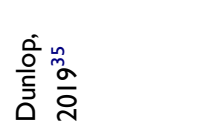 & 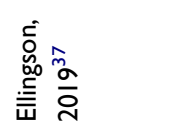 & & & 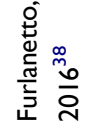 & \multicolumn{3}{|l|}{ 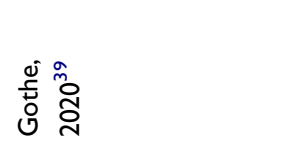 } & \multicolumn{2}{|c|}{ 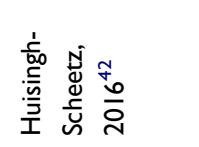 } \\
\hline
\end{tabular}




\begin{tabular}{|c|c|c|c|c|c|c|c|c|c|c|c|c|}
\hline \multicolumn{2}{|c|}{ 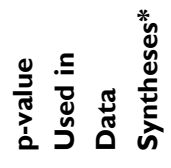 } & $\begin{array}{l}\frac{0}{\tilde{y}} \\
\frac{0}{0} \\
0\end{array}$ & 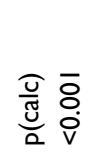 & $\begin{array}{l}\text { to } \\
\text { IIj } \\
\text { II }\end{array}$ & 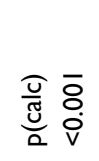 & 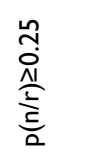 & 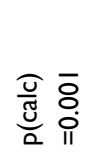 & 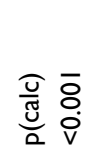 & 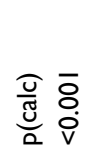 & 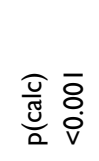 & 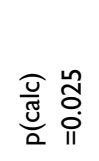 & $\begin{array}{l}\frac{0}{\tilde{J}} \\
\frac{0}{0} \\
\frac{0}{0}\end{array}$ \\
\hline \multicolumn{2}{|c|}{ 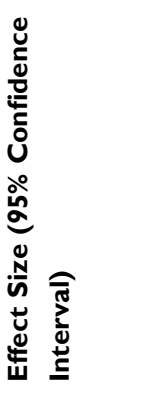 } & 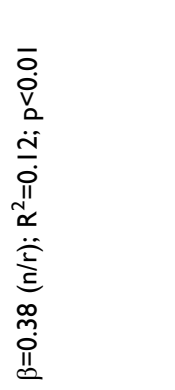 & 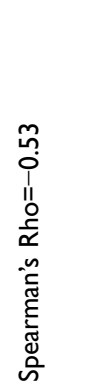 & 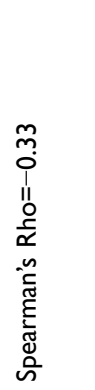 & 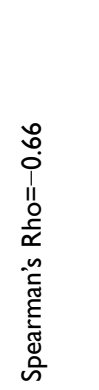 & 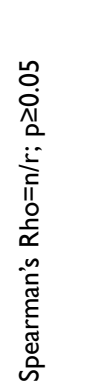 & 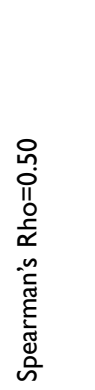 & 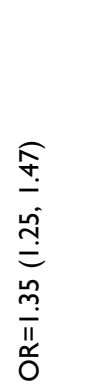 & 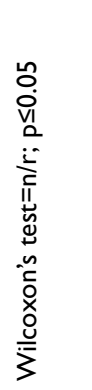 & 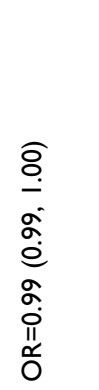 & 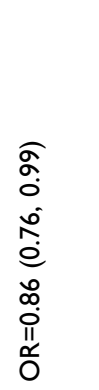 & 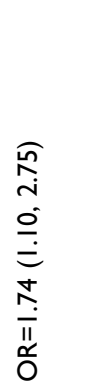 \\
\hline \multicolumn{2}{|c|}{ 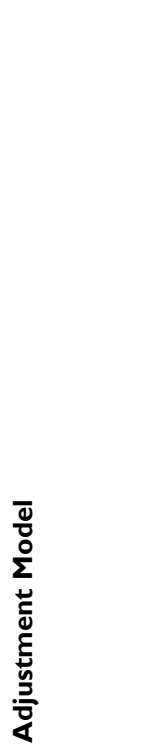 } & 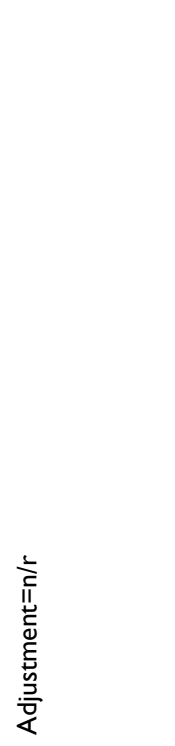 & 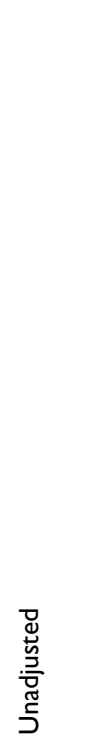 & 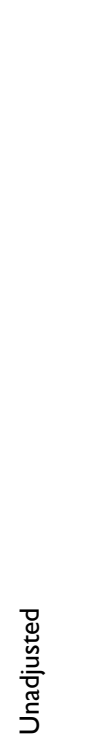 & 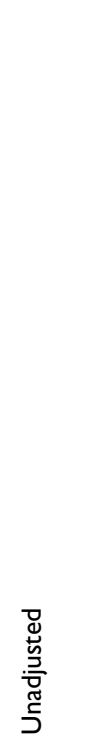 & 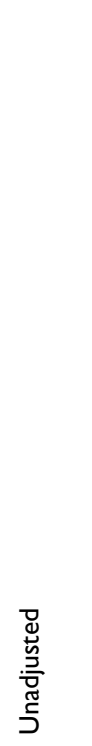 & 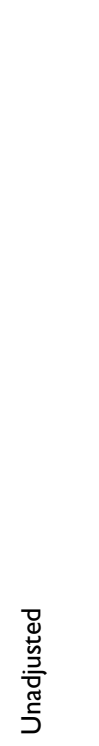 & 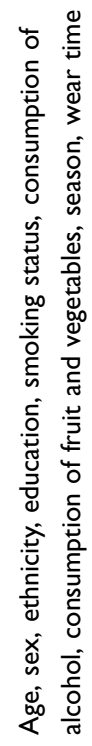 & 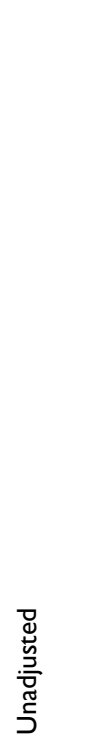 & 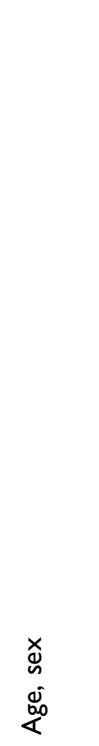 & 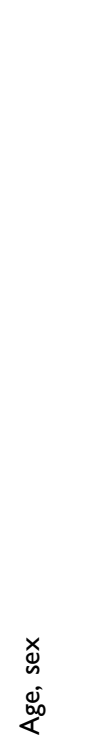 & $\begin{array}{l}\text { × } \\
0 \\
\text { s. } \\
\text { o. }\end{array}$ \\
\hline \multirow[b]{2}{*}{$\frac{1}{\stackrel{0}{a}}$} & 產 & $\begin{array}{l}\stackrel{0}{0} \\
\stackrel{0}{0} \\
\stackrel{0}{0} \\
\stackrel{0}{\circ}\end{array}$ & 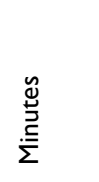 & 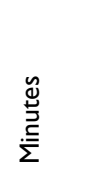 & 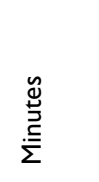 & 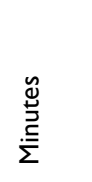 & 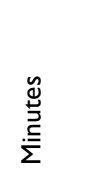 & 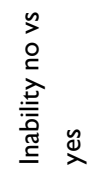 & 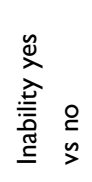 & 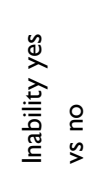 & 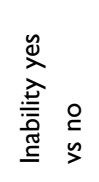 & 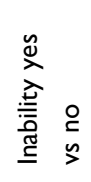 \\
\hline & 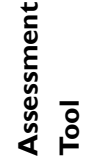 & 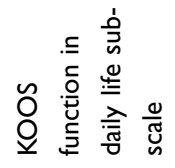 & 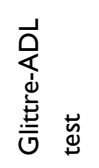 & 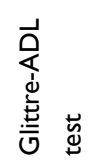 & 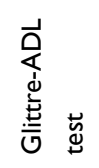 & 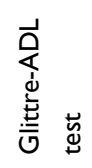 & 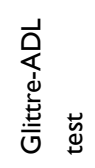 & 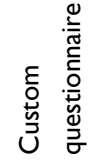 & $\begin{array}{l}\bar{a} \\
\text { ờ } \\
\text { 至 }\end{array}$ & $\begin{array}{l}\bar{a} \\
\text { ờ } \\
\text { 至 }\end{array}$ & $\begin{array}{l}\bar{\alpha} \\
\text { ờ } \\
\text { 至 }\end{array}$ & $\begin{array}{l}\bar{\alpha} \\
\text { ờ } \\
\text { 王 }\end{array}$ \\
\hline \multicolumn{2}{|c|}{ 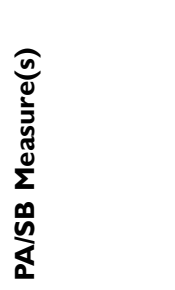 } & 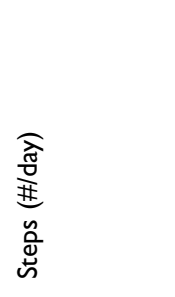 & 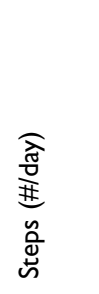 & 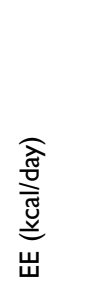 & 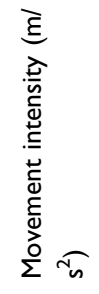 & 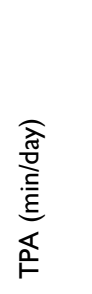 & 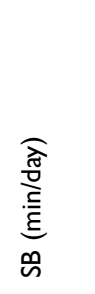 & 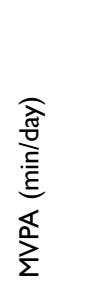 & 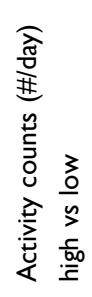 & 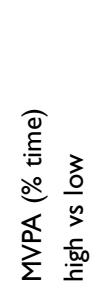 & 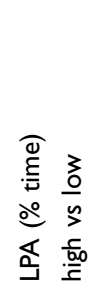 & 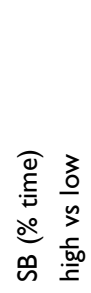 \\
\hline \multicolumn{2}{|c|}{ 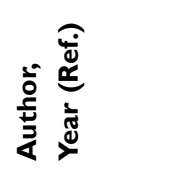 } & 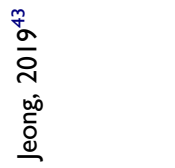 & \multicolumn{5}{|l|}{ 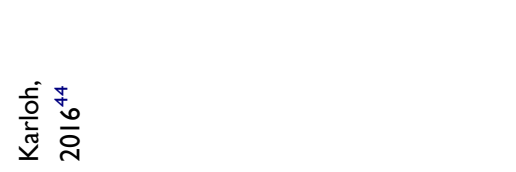 } & 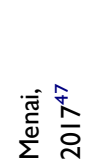 & \multicolumn{4}{|l|}{ 这 } \\
\hline
\end{tabular}




\begin{tabular}{|c|c|c|c|c|c|c|c|}
\hline 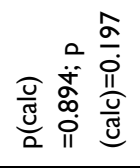 & 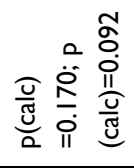 & 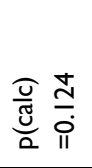 & 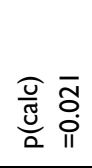 & 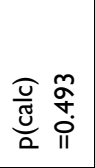 & 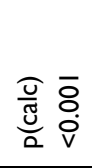 & 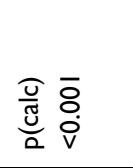 & 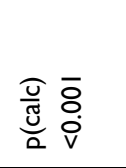 \\
\hline 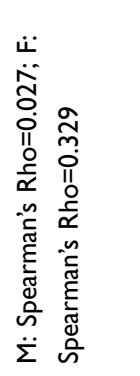 & 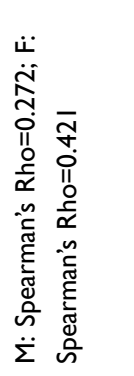 & 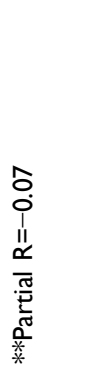 & 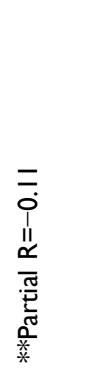 & 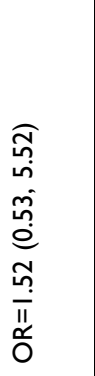 & 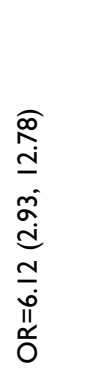 & 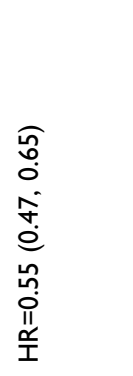 & 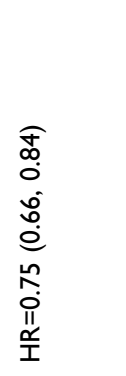 \\
\hline 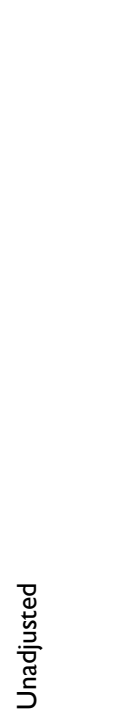 & 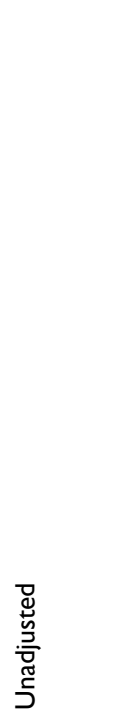 & 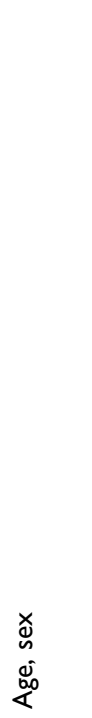 & 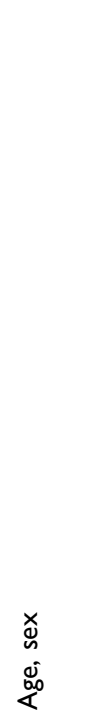 & 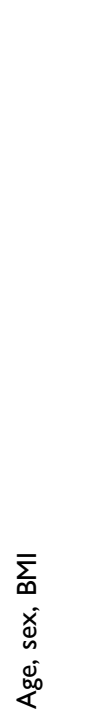 & 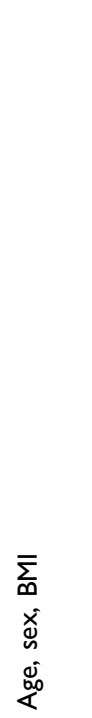 & 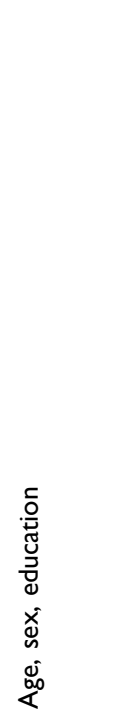 & 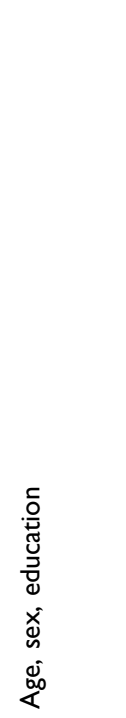 \\
\hline $\begin{array}{l}\stackrel{0}{0} \\
\stackrel{0}{0} \\
\stackrel{0}{0} \\
\stackrel{u}{0}\end{array}$ & 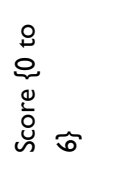 & 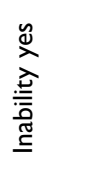 & 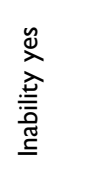 & 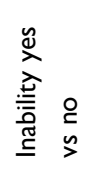 & 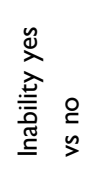 & 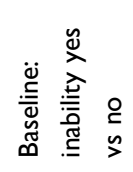 & 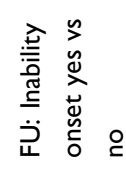 \\
\hline 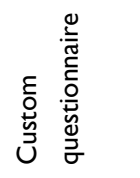 & 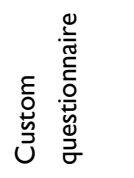 & 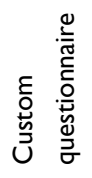 & 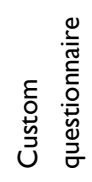 & $\begin{array}{l}\frac{0}{\tilde{J}} \\
\underline{u} \\
\stackrel{u}{U}\end{array}$ & 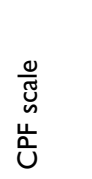 & 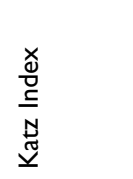 & \\
\hline 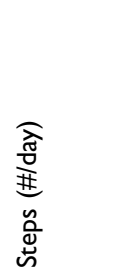 & 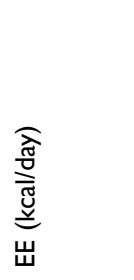 & 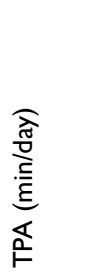 & 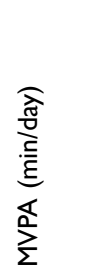 & 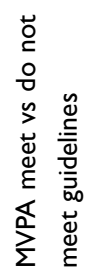 & 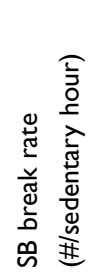 & 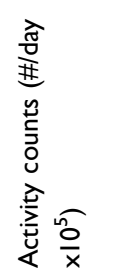 & \\
\hline $\begin{array}{l}\stackrel{d}{x} \\
\bar{D} \\
\dot{y} \\
\dot{a}\end{array}$ & & \multicolumn{2}{|l|}{ 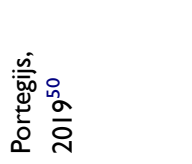 } & \multicolumn{2}{|l|}{ 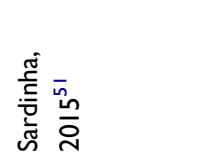 } & \multicolumn{2}{|l|}{ 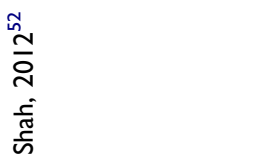 } \\
\hline
\end{tabular}




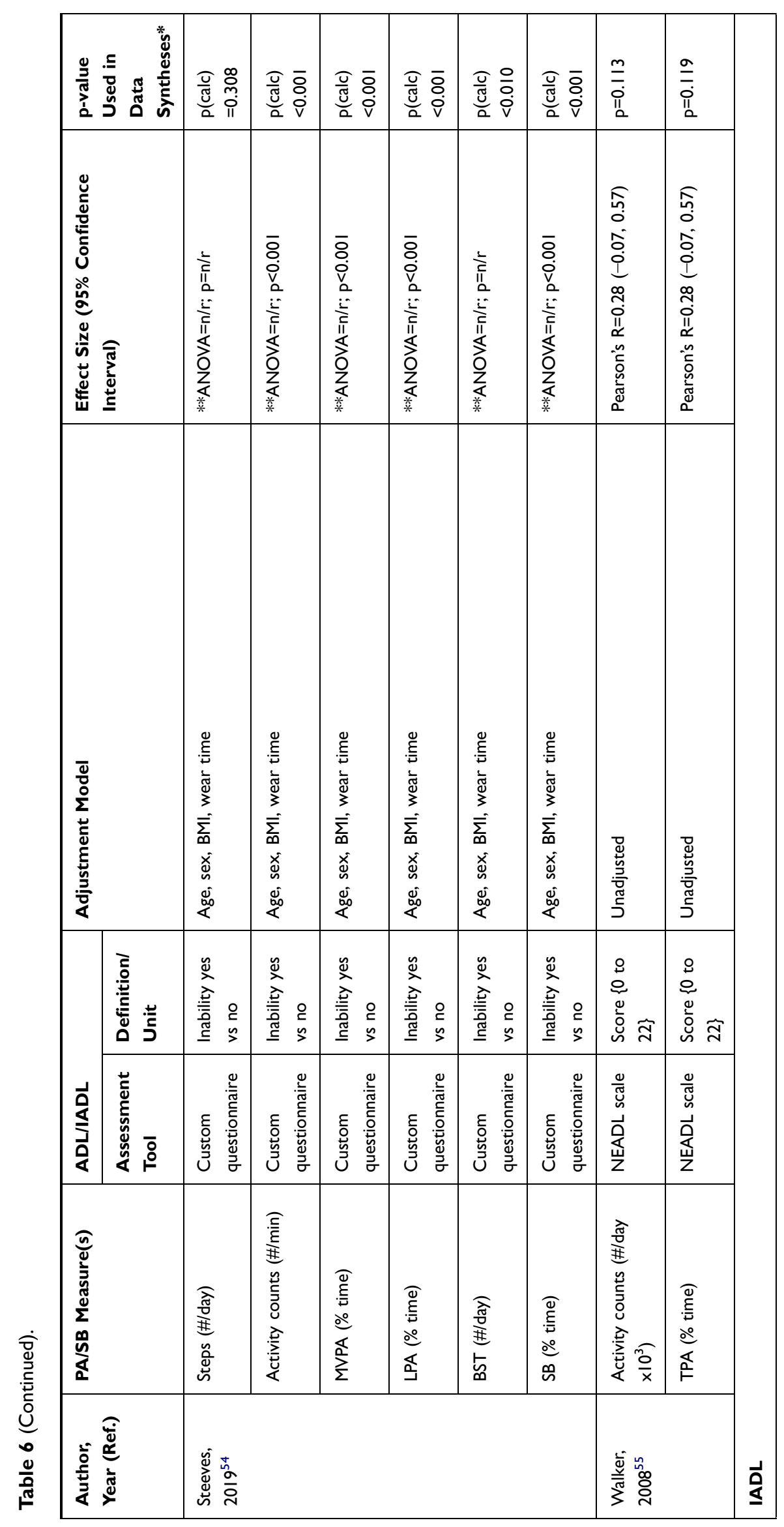




\begin{tabular}{|c|c|c|c|c|c|c|c|c|c|}
\hline 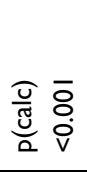 & 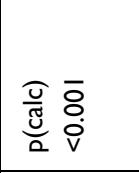 & 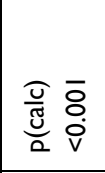 & 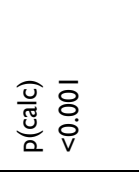 & $\begin{array}{l}\frac{\widetilde{v}}{\tilde{g}} \bar{\alpha} \\
\frac{\partial}{0}\end{array}$ & $\begin{array}{l}\frac{0}{\tilde{g}} \\
\text { 递 }\end{array}$ & 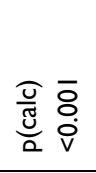 & 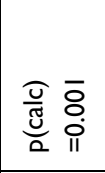 & 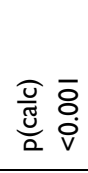 & 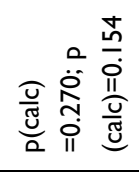 \\
\hline 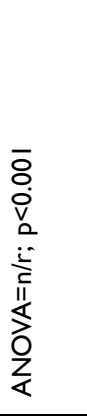 & 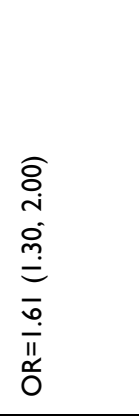 & 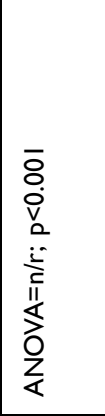 & 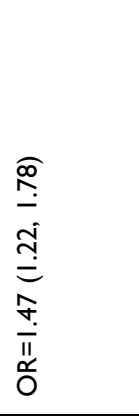 & 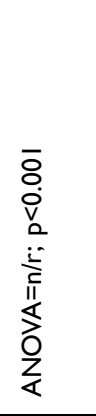 & 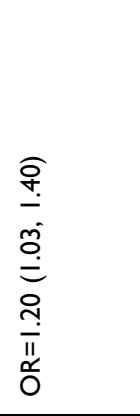 & 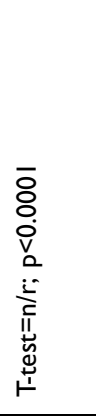 & 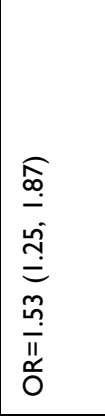 & 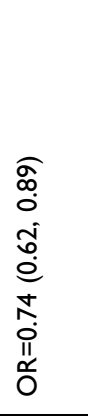 & 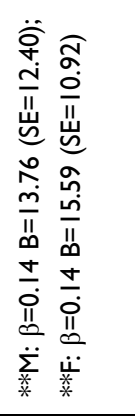 \\
\hline 离 & 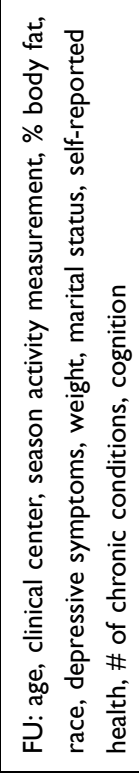 & 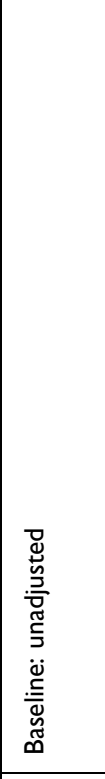 & 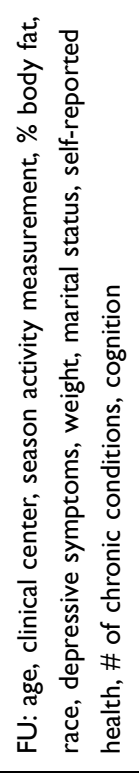 & 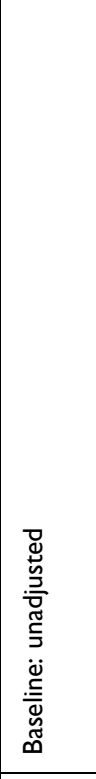 & 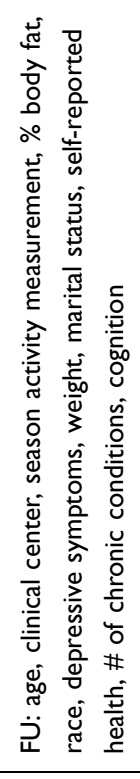 & 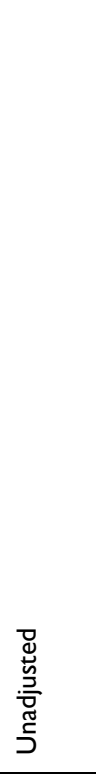 & 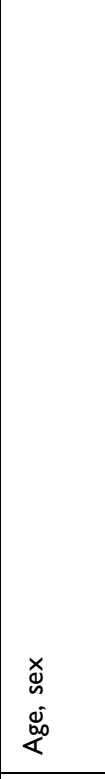 & 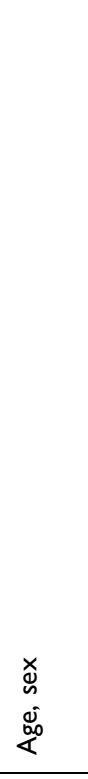 & 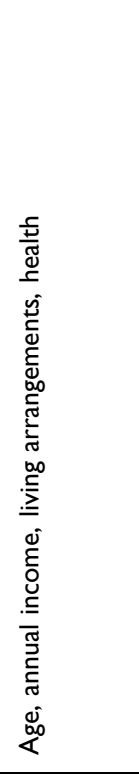 \\
\hline 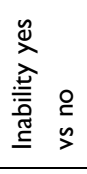 & & 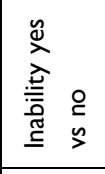 & & 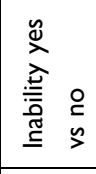 & & 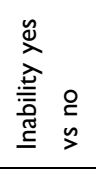 & 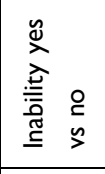 & 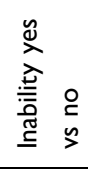 & 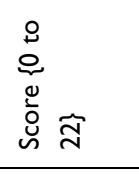 \\
\hline 递 & & 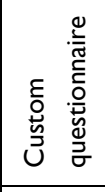 & & 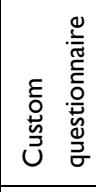 & & $\frac{\stackrel{U}{\dot{j}}}{\stackrel{i}{\Sigma}}$ & 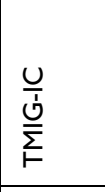 & 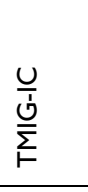 & 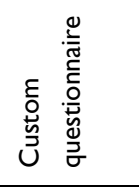 \\
\hline 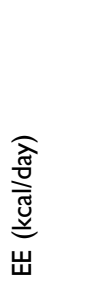 & & 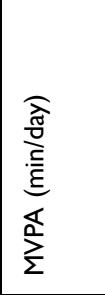 & & 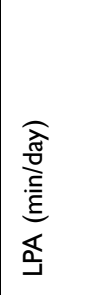 & & 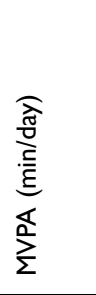 & 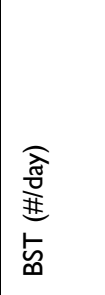 & 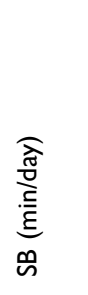 & 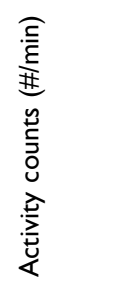 \\
\hline 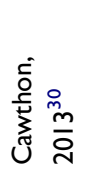 & & & & & & \multicolumn{3}{|l|}{ 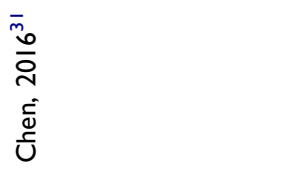 } & 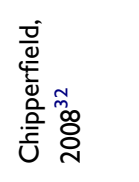 \\
\hline
\end{tabular}




\begin{tabular}{|c|c|c|c|c|c|c|c|c|c|c|c|}
\hline \multicolumn{2}{|c|}{ 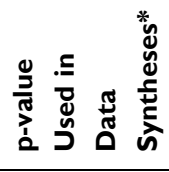 } & 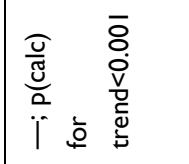 & 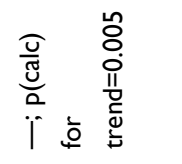 & 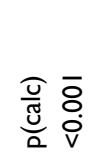 & 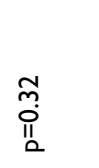 & $\begin{array}{l}\text { ô. } \\
\text { II. } \\
\text { in }\end{array}$ & $\begin{array}{l}\text { c్ } \\
\text { II } \\
\text { II }\end{array}$ & 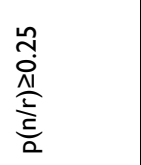 & 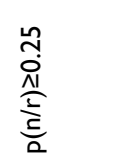 & $\begin{array}{l}\overline{0} \\
\stackrel{0}{i} \\
\text { II } \\
0\end{array}$ & $\begin{array}{l}\overline{0} \\
\text { II } \\
\text { II }\end{array}$ \\
\hline \multicolumn{2}{|c|}{ 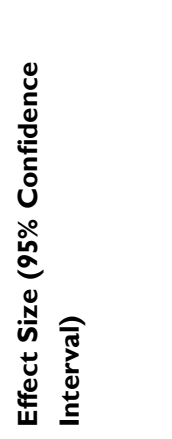 } & 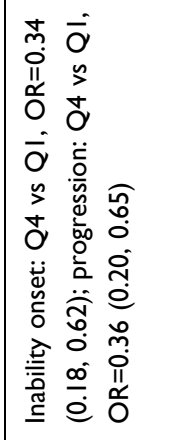 & 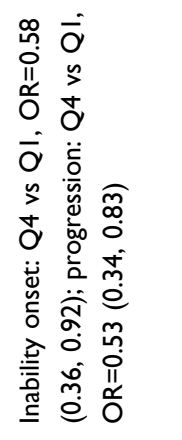 & 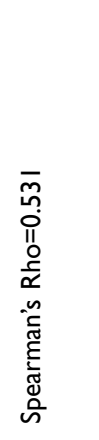 & 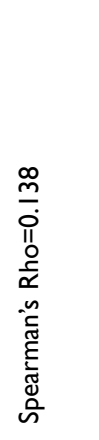 & 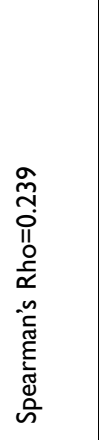 & 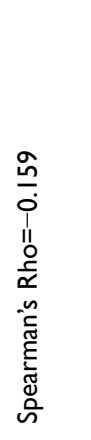 & 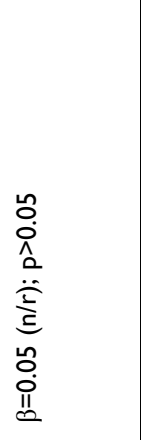 & 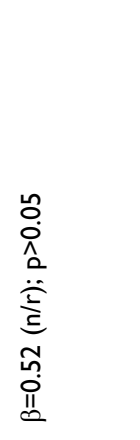 & 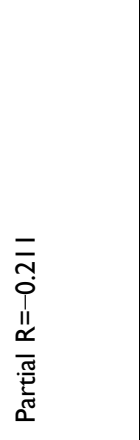 & 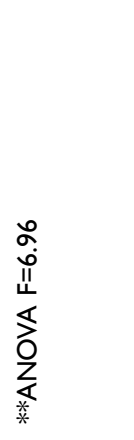 \\
\hline \multicolumn{2}{|c|}{ 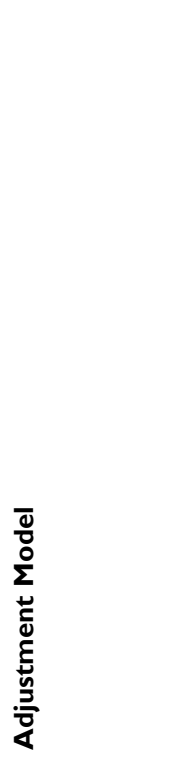 } & 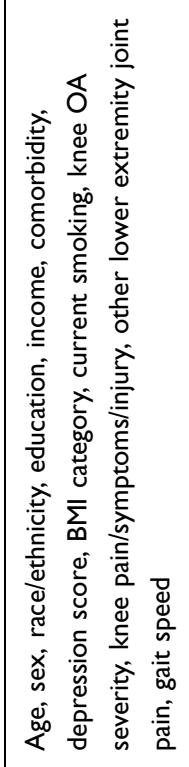 & 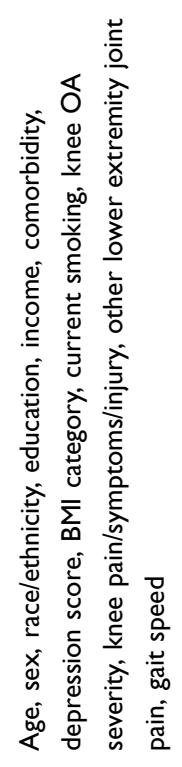 & 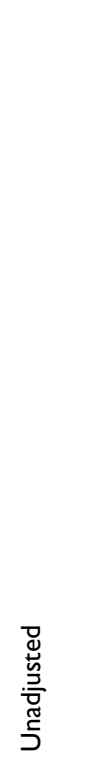 & 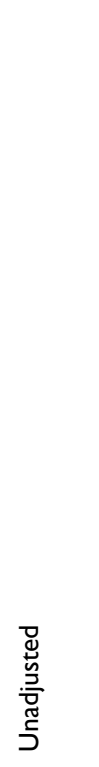 & 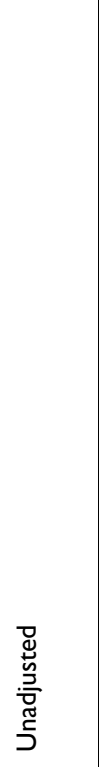 & 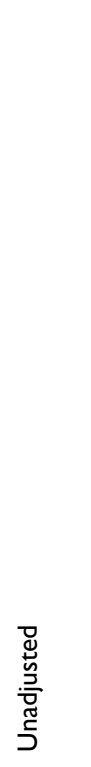 & 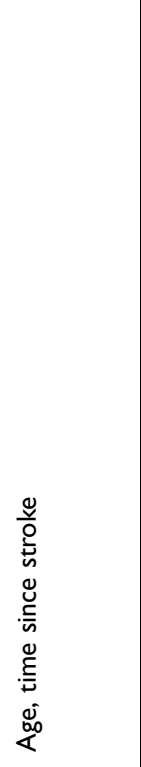 & 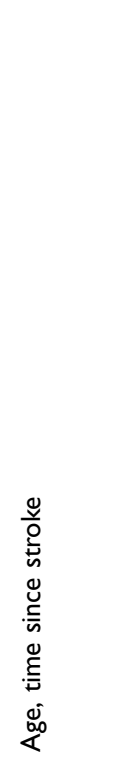 & 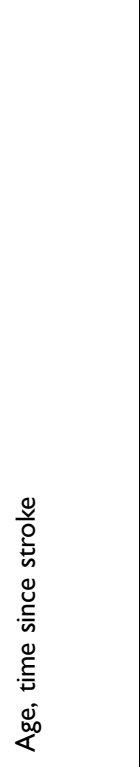 & 总 \\
\hline \multirow[b]{2}{*}{ 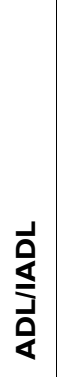 } & 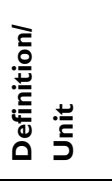 & 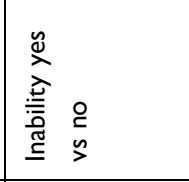 & 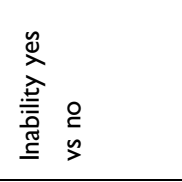 & $\begin{array}{l}\stackrel{0}{0} \\
\stackrel{0}{0} \\
\stackrel{0}{0} \\
\stackrel{\dot{n}}{m}\end{array}$ & $\begin{array}{l}\dot{0} \\
\stackrel{0}{0} \\
0 \\
\stackrel{0}{0} \\
\dot{\omega} \\
m\end{array}$ & $\begin{array}{l}\dot{0} \\
\dot{0} \\
\dot{0} \\
\dot{0} \\
\dot{u} \\
m\end{array}$ & $\begin{array}{l}\stackrel{0}{0} \\
\stackrel{0}{0} \\
0 \\
\dot{0} \\
\stackrel{n}{m}\end{array}$ & 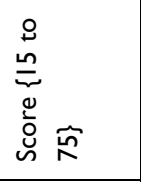 & 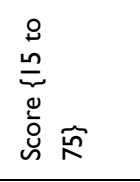 & 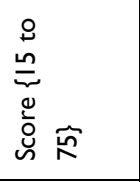 & 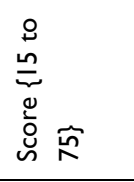 \\
\hline & 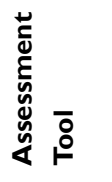 & 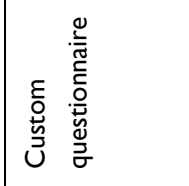 & 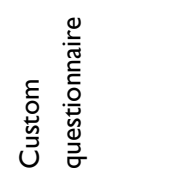 & 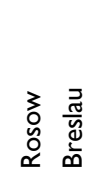 & 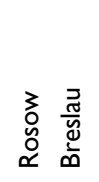 & 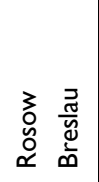 & 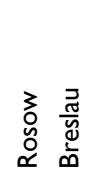 & 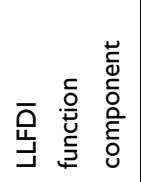 & 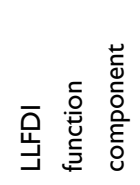 & 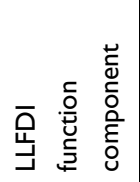 & 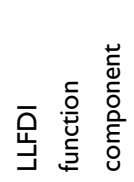 \\
\hline \multicolumn{2}{|l|}{ 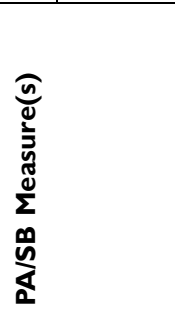 } & 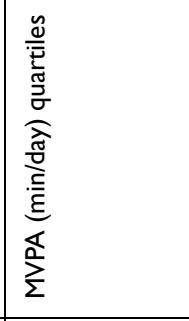 & 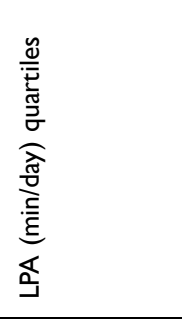 & 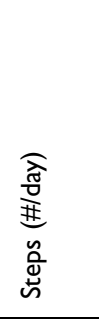 & 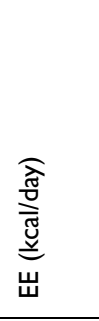 & 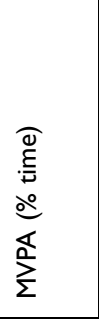 & 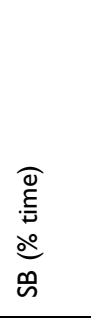 & 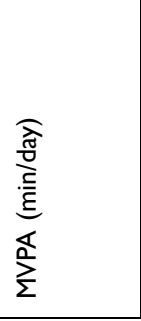 & 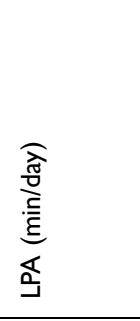 & 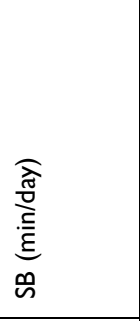 & 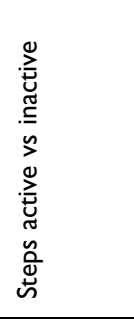 \\
\hline \multicolumn{2}{|c|}{ 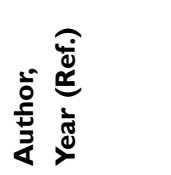 } & \multicolumn{2}{|l|}{ 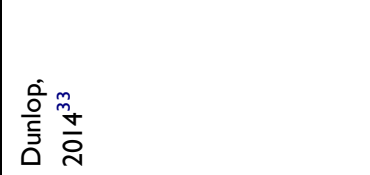 } & \multicolumn{4}{|l|}{ 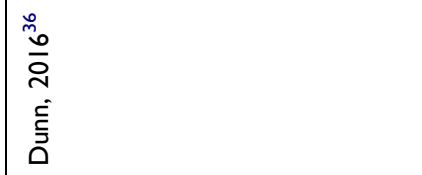 } & \multicolumn{3}{|l|}{ 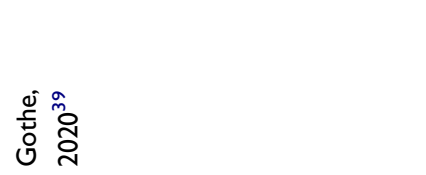 } & $\begin{array}{l}\frac{0}{0} \\
\frac{0}{2} \\
\overline{\bar{T}} \\
\text { I }\end{array}$ \\
\hline
\end{tabular}




\begin{tabular}{|c|c|c|c|c|c|c|c|c|c|}
\hline 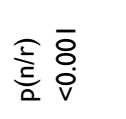 & $\begin{array}{l}\text { ¿̊. } \\
\text { iI } \\
\text { a }\end{array}$ & $\frac{0}{0}$ & $\begin{array}{l}\text { ò } \\
0 \\
\text { II } \\
a\end{array}$ & 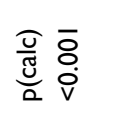 & I & 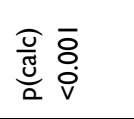 & I & 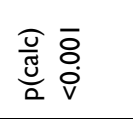 & 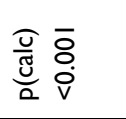 \\
\hline 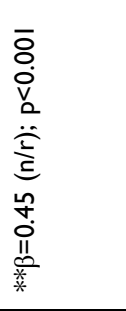 & 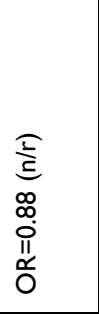 & 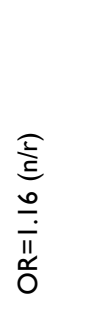 & 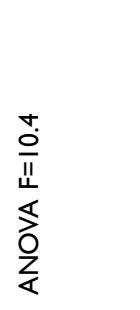 & 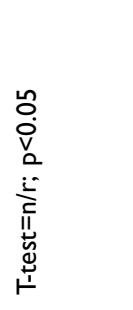 & 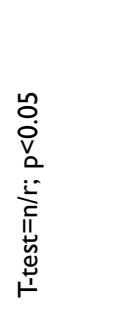 & 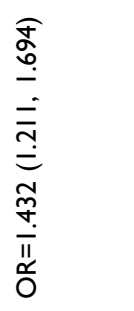 & 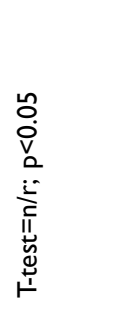 & 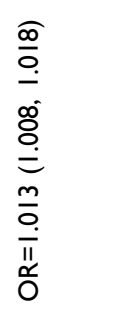 & 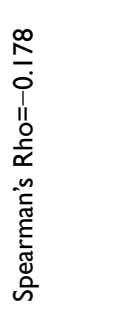 \\
\hline 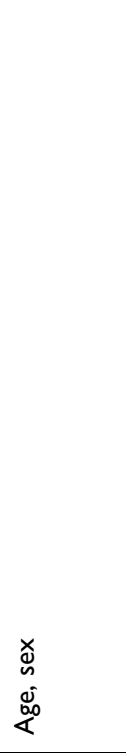 & 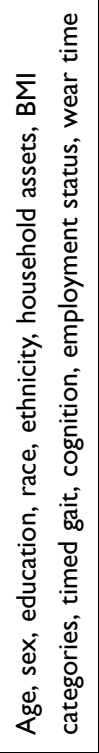 & 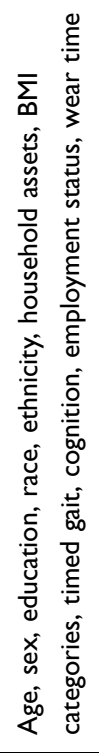 & 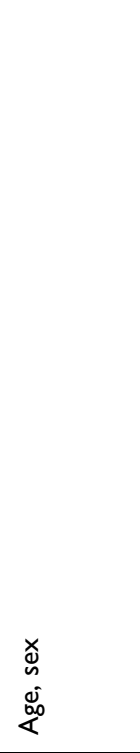 & 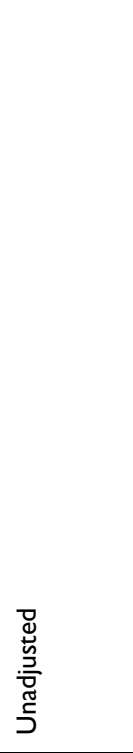 & 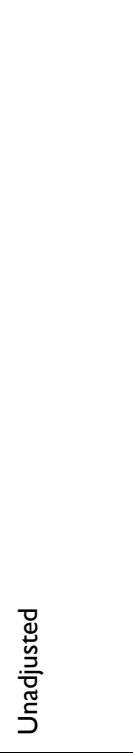 & 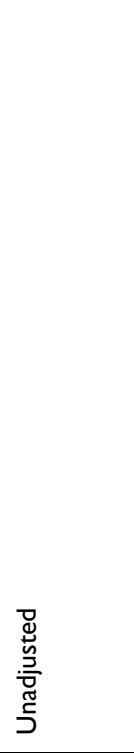 & 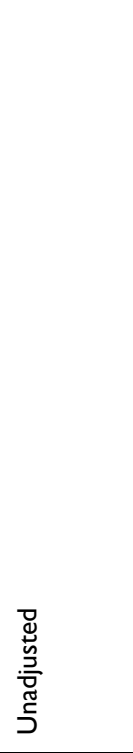 & 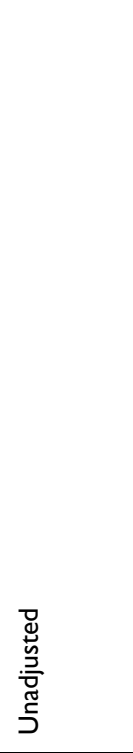 & 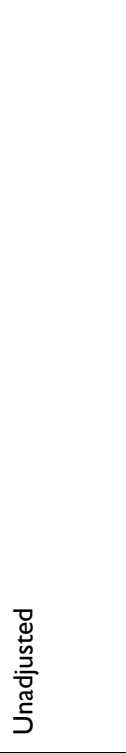 \\
\hline $\begin{array}{l}\stackrel{0}{0} \\
\stackrel{0}{0} \\
\stackrel{0}{0} \\
\stackrel{0}{0}\end{array}$ & 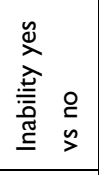 & 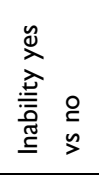 & 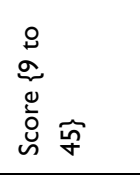 & 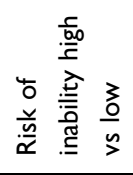 & 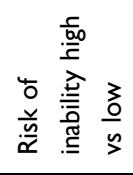 & 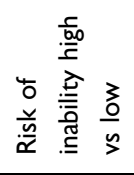 & 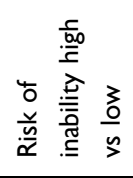 & 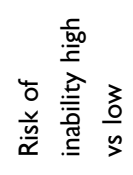 & 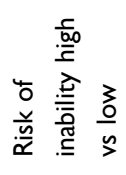 \\
\hline 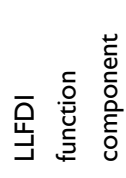 & 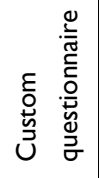 & 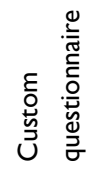 & 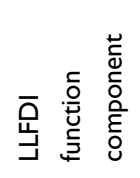 & 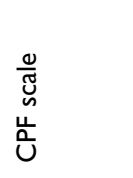 & $\begin{array}{l}\frac{0}{\tilde{J}} \\
\text { uั } \\
\stackrel{u}{U}\end{array}$ & 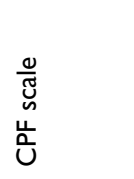 & 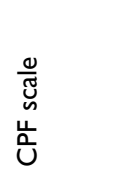 & 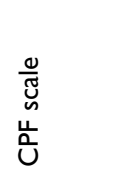 & $\begin{array}{l}\frac{0}{\tilde{J}} \\
\frac{\omega}{\Delta} \\
\tilde{U}\end{array}$ \\
\hline 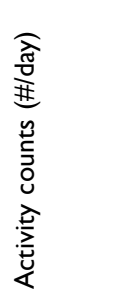 & 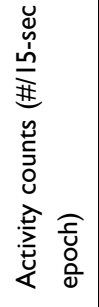 & 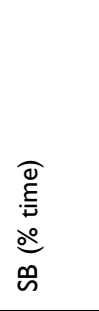 & 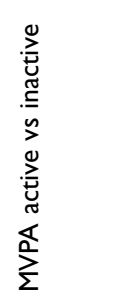 & 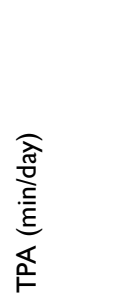 & 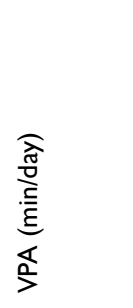 & 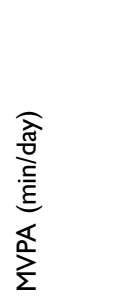 & 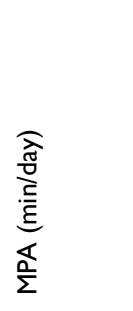 & 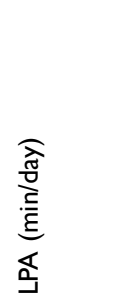 & 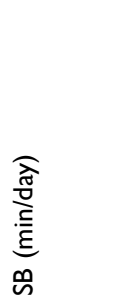 \\
\hline 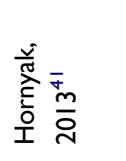 & \multicolumn{2}{|c|}{ 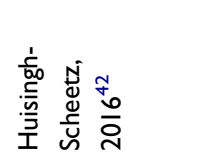 } & 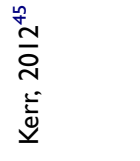 & \multicolumn{6}{|l|}{ 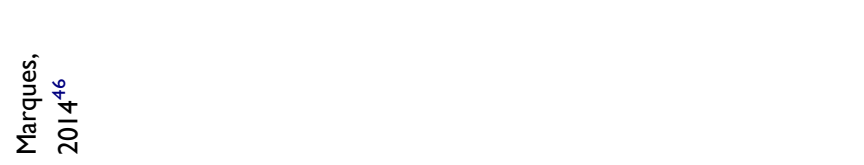 } \\
\hline
\end{tabular}




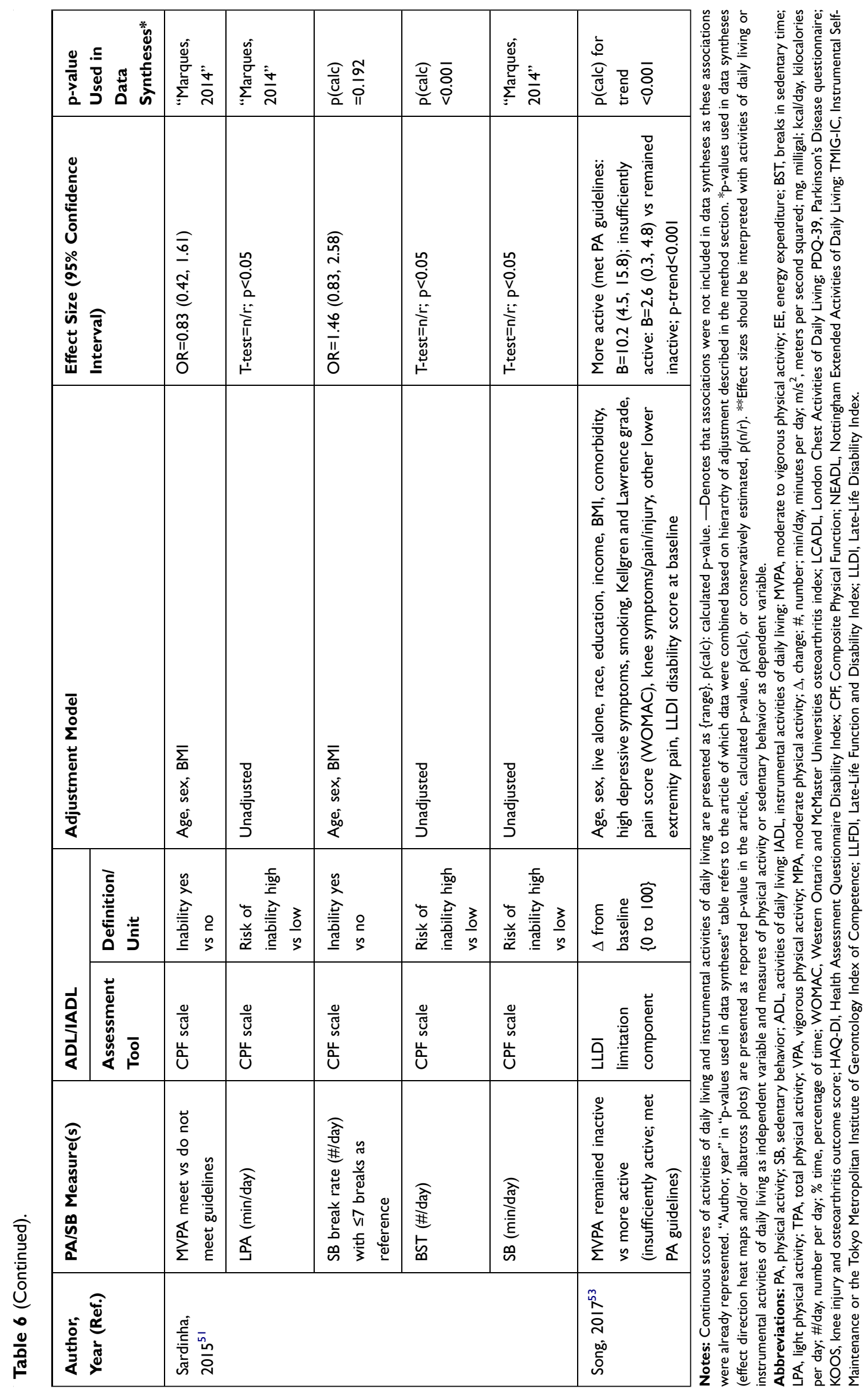


as measuring $\mathrm{ADL}$ and/or IADL, in contrast to the liberal use of keywords related to these daily-life activities throughout the literature. Despite the important advantages of measuring PA and SB objectively, accelerometers and pedometers are limited in their ability to capture loading or resistance during PA, which represents a limitation to fully characterizing PA. Our strategy in making a hierarchy of adjusted covariates to address confounding by age and sex may have suppressed the true relationship between PA and SB with ADL or IADL due to over-adjustment. While we aim to include associations only adjusted for age and sex, in some studies the closest available model includes adjustments for a range of variables beyond age and sex that may have interfered in the causal pathway, which would therefore represent over-adjustment and lower effect sizes. In all studies, except for one study that included performance-based measures of ADL, ${ }^{45}$ the ability to perform ADL and IADL was assessed by self-report of the participants themselves. Such a subjective approach in assessing ADL and IADL may lead to biases, including individual differences in self-perceived difficulty or ability to perform ADL or IADL and therefore presents a limitation. However, the ability to accurately self-assess ADL and IADL is likely easier than PA or SB given that the activities assessed are familiar and finite. Methodological challenges were also encountered in $\mathrm{PA} / \mathrm{SB}$ measures due to large variability in units, definitions, and statistical analyses used to examine the association of interest. This limitation has precluded us from performing a meta-analysis and led to alternative methods to synthesize our results.

\section{Conclusion}

Higher PA and lower SB are significantly associated with better ADL and IADL in community-dwelling older adults. Future research should, based on older adults' ability to function in daily life, aim to establish the optimal dose of PA to prevent development and progression of dependence in ADL and IADL, as well as investigating if higher PA and lower SB can recover loss of independence in one or more activities to, ultimately, design attainable lifestyle guidelines for older adults.

\section{Acknowledgments}

We sincerely thank René Otten (RO), the Vrije Universiteit librarian, for assisting the literature search of the systematic review and Luke D'Andrea (LD), Eva van der Rijt
(EvdR), Alec Tolley, and Waner Zhou (WZ) for their fruitful discussion.

\section{Funding}

This work was supported by European Union's Horizon 2020 research (No. 675003); and innovation programme (No. 689238).

\section{Disclosure}

The authors report no conflicts of interest in this work.

\section{References}

1. Caspersen CJ, Powell KE, Christenson GM. Physical activity, exercise, and physical fitness: definitions and distinctions for healthrelated research. Public Health Rep. 1985;100(2):126-131.

2. Vogel T, Brechat PH, Lepretre PM, Kaltenbach G, Berthel M, Lonsdorfer J. Health benefits of physical activity in older patients: a review. Int J Clin Pract. 2009;63(2):303-320.

3. Hallal PC, Andersen LB, Bull FC, et al. Global physical activity levels: surveillance progress, pitfalls, and prospects. Lancet. 2012; 380(9838):247-257.

4. Gomes M, Figueiredo D, Teixeira L, et al. Physical inactivity among older adults across Europe based on the SHARE database. Age Ageing. 2017;46(1):71-77.

5. Milanovic Z, Pantelic S, Trajkovic N, Sporis G, Kostic R, James N. Age-related decrease in physical activity and functional fitness among elderly men and women. Clin Interv Aging. 2013;8:549-556.

6. Tremblay MS, Aubert S, Barnes JD, et al. Sedentary Behavior Research Network (SBRN) - Terminology Consensus Project process and outcome. Int J Behav Nutr Phys Act. 2017;14(1):75.

7. van der Ploeg HP, Hillsdon M. Is sedentary behaviour just physical inactivity by another name? Int J Behav Nutr Phys Act. 2017;14 (1): 142 .

8. Bowden Davies KA, Pickles S, Sprung VS, et al. Reduced physical activity in young and older adults: metabolic and musculoskeletal implications. Ther Adv Endocrinol Metab. 2019;10:2042018819888824.

9. Santos DA, Silva AM, Baptista F, et al. Sedentary behavior and physical activity are independently related to functional fitness in older adults. Exp Gerontol. 2012;47(12):908-912.

10. Wheeler MJ, Dempsey PC, Grace MS, et al. Sedentary behavior as a risk factor for cognitive decline? A focus on the influence of glycemic control in brain health. Alzheimers Dement. 2017;3(3):291-300.

11. Millan-Calenti JC, Tubio J, Pita-Fernandez S, et al. Prevalence of functional disability in activities of daily living (ADL), instrumental activities of daily living (IADL) and associated factors, as predictors of morbidity and mortality. Arch Gerontol Geriatr. 2010;50(3):306-310.

12. Paterson DH, Warburton DE. Physical activity and functional limitations in older adults: a systematic review related to Canada's Physical Activity Guidelines. Int J Behav Nutr Phys Act. 2010;7:38.

13. Tak E, Kuiper R, Chorus A, Hopman-Rock M. Prevention of onset and progression of basic ADL disability by physical activity in community dwelling older adults: a meta-analysis. Ageing Res Rev. 2013;12(1):329-338.

14. Scher LML, Guarda F, Barros MG, Chen Z, Anton S. Sedentary time and disability in older adults: a systematic review and meta-analysis. J Aging Sci. 2019;7:1-9.

15. Ryan DJ, Wullems JA, Stebbings GK, Morse CI, Stewart CE, Onambele-Pearson GL. Reliability and validity of the international physical activity questionnaire compared to calibrated accelerometer cut-off points in the quantification of sedentary behaviour and physical activity in older adults. PLoS One. 2018;13(4):e0195712. 
16. Tudor-Locke CE, Myers AM. Challenges and opportunities for measuring physical activity in sedentary adults. Sports Med. 2001;31 (2):91-100.

17. Silfee VJ, Haughton CF, Jake-Schoffman DE, et al. Objective measurement of physical activity outcomes in lifestyle interventions among adults: a systematic review. Prev Med Rep. 2018;11:74-80.

18. Moher D, Liberati A, Tetzlaff J, Altman DG, Group P. Preferred reporting items for systematic reviews and meta-analyses: the PRISMA statement. Ann Intern Med. 2009;151(4):264-269, W264.

19. Ouzzani M, Hammady H, Fedorowicz Z, Elmagarmid A. Rayyan-a web and mobile app for systematic reviews. Syst Rev. 2016;5(1):210.

20. Wells G, Shea B, O'Connell D, et al. The Newcastle-Ottawa Scale (NOS) for assessing the quality of non-randomized studies in metaanalysis. Ottawa Hosp Res Inst. 2000.

21. Thomson HJ, Thomas S. The effect direction plot: visual display of non-standardised effects across multiple outcome domains. Res Synth Methods. 2013;4(1):95-101.

22. Harrison S, Jones HE, Martin RM, Lewis SJ, Higgins JPT. The albatross plot: a novel graphical tool for presenting results of diversely reported studies in a systematic review. Res Synth Methods. 2017;8(3):281-289.

23. Campbell M, McKenzie JE, Sowden A, et al. Synthesis without metaanalysis (SWiM) in systematic reviews: reporting guideline. BMJ. 2020;368:16890.

24. Altman DG, Bland JM. How to obtain the P value from a confidence interval. BMJ. 2011;343:d2304.

25. Ramsey KA, Rojer AGM, D'Andrea L, et al. The association of objectively measured physical activity and sedentary behavior with skeletal muscle strength and muscle power in older adults: a systematic review and meta-analysis. Ageing Res Rev. 2021;101266.

26. Kingston A, Collerton J, Davies K, Bond J, Robinson L, Jagger C. Losing the ability in activities of daily living in the oldest old: a hierarchic disability scale from the Newcastle $85+$ study. PLoS One. 2012;7(2):e31665.

27. Balogun S, Scott D, Cicuttini F, Jones G, Aitken D. Longitudinal study of the relationship between physical activity and knee pain and functional limitation in community-dwelling older adults. Arch Gerontol Geriatr. 2020;90:104101.

28. Barriga S, Rodrigues F, Barbara C. Factors that influence physical activity in the daily life of male patients with chronic obstructive pulmonary disease. Rev Port Pneumol. 2014;20(3):131-137.

29. Bielemann RM, LaCroix AZ, Bertoldi AD, et al. Objectively measured physical activity reduces the risk of mortality among Brazilian older adults. $J$ Am Geriatr Soc. 2020;68(1):137-146.

30. Blodgett J, Theou O, Kirkland S, Andreou P, Rockwood K. The association between sedentary behaviour, moderate-vigorous physical activity and frailty in NHANES cohorts. Maturitas. 2015;80(2):187-191.

31. Cawthon PM, Blackwell TL, Cauley JA, et al. Objective assessment of activity, energy expenditure, and functional limitations in older men: the Osteoporotic Fractures in Men study. J Gerontol a Biol Sci Med Sci. 2013;68(12):1518-1524.

32. Chen T, Narazaki K, Haeuchi Y, Chen S, Honda T, Kumagai S. Associations of sedentary time and breaks in sedentary time with disability in instrumental activities of daily living in communityDwelling older adults. $J$ Phys Act Health. 2016;13(3):303-309.

33. Chipperfield JG, Newall NE, Chuchmach LP, Swift AU, Haynes TL. Differential determinants of men's and women's everyday physical activity in later life. J Gerontol B Psychol Sci Soc Sci. 2008;63(4): S211-S218.

34. Dunlop DD, Song J, Semanik PA, et al. Relation of physical activity time to incident disability in community dwelling adults with or at risk of knee arthritis: prospective cohort study. BMJ. 2014;348: g2472.

35. Dunlop DD, Song J, Arnston EK, et al. Sedentary time in US older adults associated with disability in activities of daily living independent of physical activity. $J$ Phys Act Health. 2015;12(1):93-101.
36. Dunlop DD, Song J, Hootman JM, et al. One hour a week: moving to prevent disability in adults with lower extremity joint symptoms. $\mathrm{Am}$ J Prev Med. 2019;56(5):664-672.

37. Dunn MA, Josbeno DA, Schmotzer AR, et al. The gap between clinically assessed physical performance and objective physical activity in liver transplant candidates. Liver Transpl. 2016;22(10):13241332.

38. Ellingson LD, Zaman A, Stegemoller EL. Sedentary behavior and quality of life in individuals with Parkinson's disease. Neurorehabil Neural Repair. 2019;33(8):595-601.

39. Furlanetto KC, Pinto IF, Sant'Anna T, Hernandes NA, Pitta F. Profile of patients with chronic obstructive pulmonary disease classified as physically active and inactive according to different thresholds of physical activity in daily life. Braz J Phys Ther. 2016;20(6):517-524.

40. Gothe NP, Bourbeau K. Associations between physical activity intensities and physical function in stroke survivors. Am J Phys Med Rehabil. 2020;99(8):733-738.

41. Hall KS, McAuley E. Individual, social environmental and physical environmental barriers to achieving 10000 steps per day among older women. Health Educ Res. 2010;25(3):478-488.

42. Hornyak V, Brach JS, Wert DM, Hile E, Studenski S, VanSwearingen JM. What is the relation between fear of falling and physical activity in older adults? Arch Phys Med Rehabil. 2013;94(12):2529-2534.

43. Huisingh-Scheetz MJ, Kocherginsky M, Magett E, Rush P, Dale W, Waite L. Relating wrist accelerometry measures to disability in older adults. Arch Gerontol Geriatr. 2016;62:68-74.

44. Jeong JN, Kim SH, Park KN. Relationship between objectively measured lifestyle factors and health factors in patients with knee osteoarthritis: the STROBE Study. Medicine. 2019;98(26):e16 060 .

45. Karloh M, Araujo CL, Gulart AA, Reis CM, Steidle LJ, Mayer AF. The Glittre-ADL test reflects functional performance measured by physical activities of daily living in patients with chronic obstructive pulmonary disease. Braz J Phys Ther. 2016;20(3):223-230.

46. Kerr J, Marshall S, Godbole S, et al. The relationship between outdoor activity and health in older adults using GPS. Int $J$ Environ Res Public Health. 2012;9(12):4615-4625.

47. Marques EA, Baptista F, Santos DA, Silva AM, Mota J, Sardinha LB. Risk for losing physical independence in older adults: the role of sedentary time, light, and moderate to vigorous physical activity. Maturitas. 2014;79(1):91-95.

48. Menai M, van Hees VT, Elbaz A, Kivimaki M, Singh-Manoux A, Sabia S. Accelerometer assessed moderate-to-vigorous physical activity and successful ageing: results from the Whitehall II study. Sci Rep. 2017;8:45772.

49. Ortlieb S, Gorzelniak L, Nowak D, et al. Associations between multiple accelerometry-assessed physical activity parameters and selected health outcomes in elderly people-results from the KORAage study. PLoS One. 2014;9(11):e111206.

50. Pes GM, Dore MP, Errigo A, Poulain M. Analysis of physical activity among free-living nonagenarians from a Sardinian longevous population. J Aging Phys Act. 2018;26(2):254-258.

51. Portegijs E, Karavirta L, Saajanaho M, Rantalainen T, Rantanen T. Assessing physical performance and physical activity in large population-based aging studies: home-based assessments or visits to the research center? BMC Public Health. 2019;19(1):1570.

52. Sardinha LB, Ekelund U, Dos Santos L, Cyrino ES, Silva AM, Santos DA. Breaking-up sedentary time is associated with impairment in activities of daily living. Exp Gerontol. 2015;72:57-62.

53. Shah RC, Buchman AS, Leurgans S, Boyle PA, Bennett DA. Association of total daily physical activity with disability in community-dwelling older persons: a prospective cohort study. $B M C$ Geriatr. 2012;12:63.

54. Song J, Gilbert AL, Chang RW, et al. Do inactive older adults who increase physical activity experience less disability: evidence from the osteoarthritis initiative. J Clin Rheumatol. 2017;23(1):26-32. 
55. Steeves JA, Shiroma EJ, Conger SA, Van Domelen D, Harris TB. Physical activity patterns and multimorbidity burden of older adults with different levels of functional status: NHANES 2003-2006. Disabil Health J. 2019;12(3):495-502.

56. Walker PP, Burnett A, Flavahan PW, Calverley PM. Lower limb activity and its determinants in COPD. Thorax. 2008;63(8):683-689.

57. Lee PG, Jackson EA, Richardson CR. Exercise prescriptions in older adults. Am Fam Physician. 2017;95(7):425-432.

58. Zhang Y, Zhang Y, Du S, Wang Q, Xia H, Sun R. Exercise interventions for improving physical function, daily living activities and quality of life in community-dwelling frail older adults: a systematic review and meta-analysis of randomized controlled trials. Geriatr Nurs. 2020;41(3):261-273.

59. Boyle PA, Cohen RA, Paul R, Moser D, Gordon N. Cognitive and motor impairments predict functional declines in patients with vascular dementia. Int J Geriatr Psychiatry. 2002;17(2):164-169.

60. Sanchez-Garcia S, Garcia-Pena C, Ramirez-Garcia E, MorenoTamayo K, Cantu-Quintanilla GR. Decreased autonomy in community-Dwelling older adults. Clin Interv Aging. 2019;14:2041-2053.
61. Stamm TA, Pieber K, Crevenna R, Dorner TE. Impairment in the activities of daily living in older adults with and without osteoporosis, osteoarthritis and chronic back pain: a secondary analysis of population-based health survey data. BMC Musculoskelet Disord. 2016;17:139

62. Luppa M, Luck T, Weyerer S, Konig HH, Brahler E, Riedel-Heller SG. Prediction of institutionalization in the elderly. A systematic review. Age Ageing. 2010;39(1):31-38.

63. van Lummel RC, Walgaard S, Pijnappels M, et al. Physical performance and physical activity in older adults: associated but separate domains of physical function in old age. PLoS One. 2015;10(12): e0144048.

64. Garcia-Esquinas E, Ortola R, Martinez-Gomez D, et al. Causal effects of physical activity and sedentary behaviour on health deficits accumulation in older adults. Int $J$ Epidemiol. 2020;30:852-865.

65. Washburn RA. Assessment of physical activity in older adults. Res $Q$ Exerc Sport. 2000;71(Suppl 2):79-87.
Clinical Interventions in Aging

\section{Publish your work in this journal}

Clinical Interventions in Aging is an international, peer-reviewed journal focusing on evidence-based reports on the value or lack thereof of treatments intended to prevent or delay the onset of maladaptive correlates of aging in human beings. This journal is indexed on PubMed Central, MedLine, CAS, Scopus and the Elsevier
Dovepress

Bibliographic databases. The manuscript management system is completely online and includes a very quick and fair peer-review system, which is all easy to use. Visit http://www.dovepress.com/ testimonials.php to read real quotes from published authors. 